United States

Department of

Agriculture

Forest Service

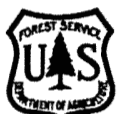

Southern Research

Station

Resource Bulletin

SRS-6
Forest Statistics for Florida, 1995

\author{
Mark J. Brown
}

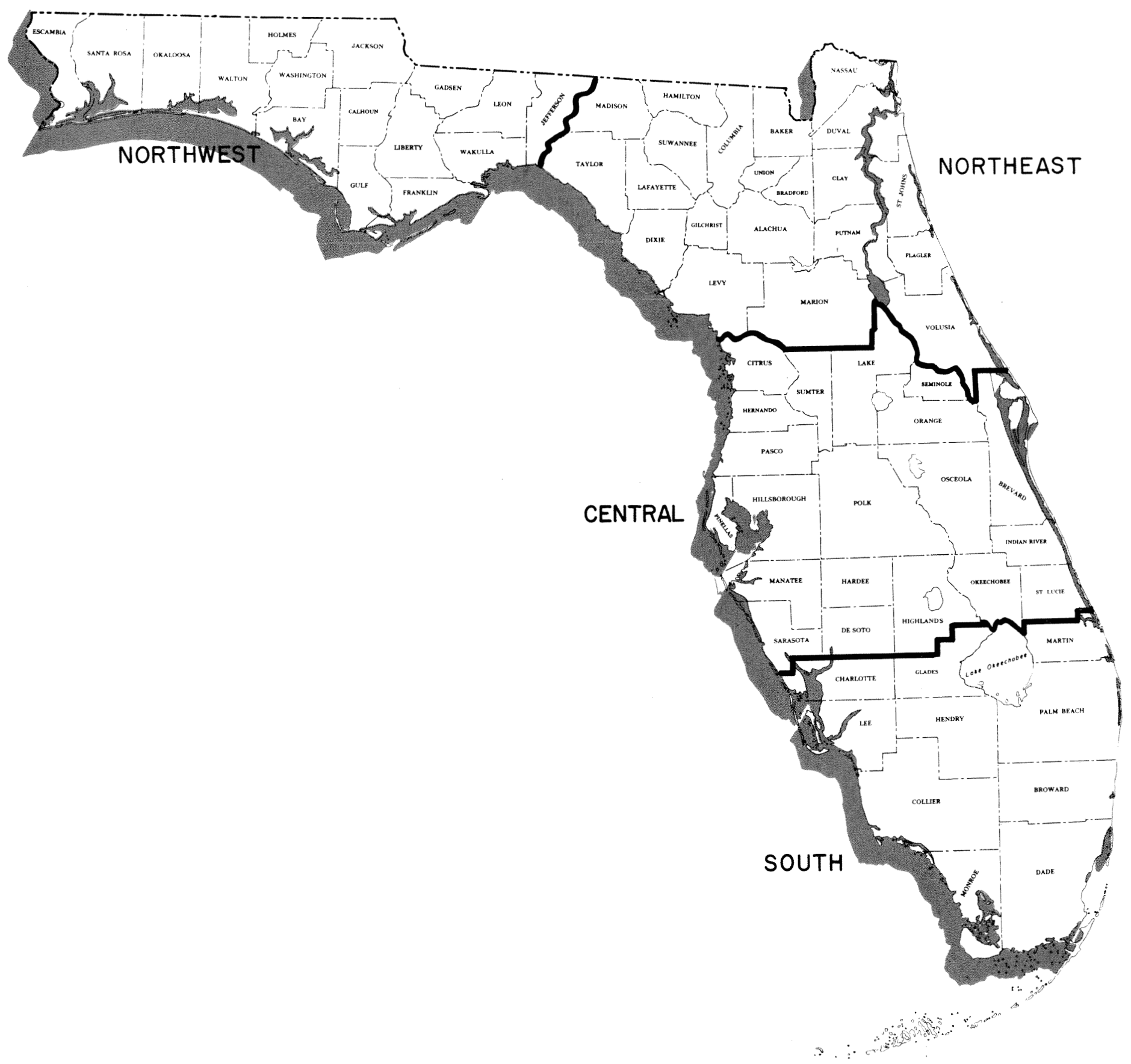


April 1996

Southern Research Station

P.O. Box 2680

Asheville, NC 28802 


\section{Forest Statistics for Florida, 1995}

Mark J. Brown, Resource Analyst

Forest Inventory and Analysis

Asheville, North Carolina 


\section{Foreword}

This report highlights the principal findings of the seventh forest survey of Florida. Field work began in June 1993 and was completed in May 1995. Six previous surveys, completed in 1936,1949,1959,1970,1980, and 1987 provide statistics for measuring changes and trends over the past 59 years. This report primarily emphasizes the changes and trends since 1987.

Periodic surveys of forest resources are authorized by the Forest and Rangeland Renewable Resources Research Act of 1978. These surveys are a continuing, nationwide undertaking by the Regional Experiment Stations of the USDA Forest Service. In the Southern United States, these surveys are conducted by two Forest Inventory and Analysis (FIA) Research Work Units at the Southern Research Station, Asheville, NC. The two FIA units, one located in Starkville, MS, and the other in Asheville, NC, are responsible for inventories of 13 Southern States and the Commonwealth of Puerto Rico. The primary objective of these surveys is to periodically inventory and evaluate all forest and related resources. These multiresource data help provide a basis for formulating forest policies and programs and for the orderly development and use of the resources. This report deals only with the extent and condition of forest land, associated timber volumes, and rates of timber growth, mortality, and removals.

Additional information about any aspect of this survey may be obtained from:

Forest Inventory and Analysis

Southern Research Station

P.O. Box 2680

Asheville, NC 28802

Phone: $704-257-4350$

\section{Acknowledgments}

The Southern Research Station gratefully acknowledges the cooperation and assistance provided by the Division of Forestry, Florida Department of Agriculture and Consumer Services in collecting field data. Appreciation is also expressed for the excellent cooperation of other public agencies, forest industry, and other private landowners in providing information and access to the sample locations.

The following members of the FIA staff collected the field data:

\author{
George Baraket \\ Mark Bloomfield \\ Robert Bowman \\ Mike Buchanan \\ Rachel Buchanan \\ Kevin Caswell \\ Robert Clark \\ Sarah Combs \\ Wesley Floyd
}

\author{
Jack Hampton \\ Ben Koontz \\ Dave Lambert \\ James McCall \\ Bill Moody \\ Mike Norris \\ Michael O'Quinn \\ David Phlegor \\ Terry Riley
}

\author{
Byron Rominger \\ Edward Scott \\ John Simpson \\ Greg Smith \\ Odell Tucker \\ Warren Tucker \\ James Twaroski \\ James Wiggins
}




\section{Contents}

Page

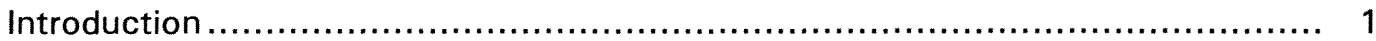

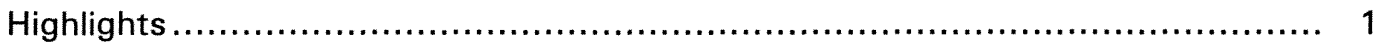

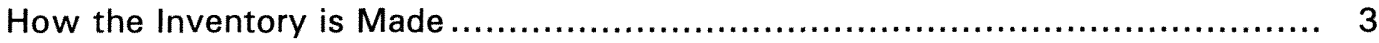

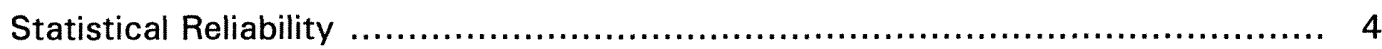

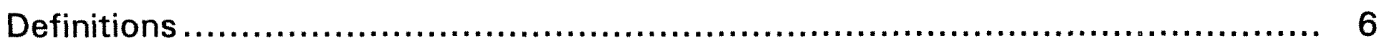

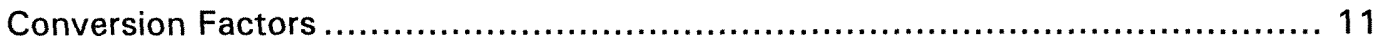

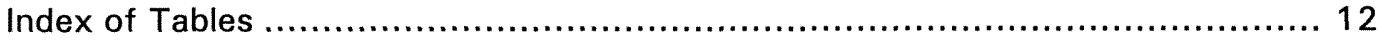

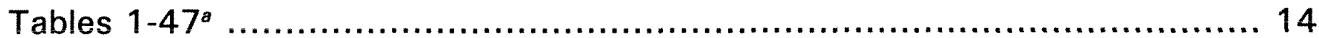

${ }^{8}$ All tables in this report are available in Microsoft ${ }^{1}$ Excel workbook files. These files will be supplied, upon

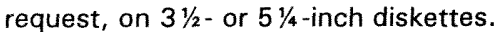

The use of trade or firm names in this publication is for reader information and does not imply endorsement by the U.S. Department of Agriculture of any product or service. 


\section{Introduction}

This report summarizes results from a 1995 inventory of the forest resources of Florida. Current estimates of forest area, related attributes, and timber volumes are presented and compared with earlier estimates. Timber volumes reported in previous bulletins have been adjusted for valid comparisons with current assessments. Average annual rates of growth, removals, and mortality since the previous inventory in 1987 have been summarized.

\section{Highlights}

\section{Since the sixth inventory of Florida's forest resources was completed in 1987-}

- area of timberland decreased more than 2 percent to less than 14.7 million acres. The 332,000-acre net loss resulted from the diversion of 916,000 acres of timberland to other land uses, less 584,000 acres returning to timberland. More than one-half of the diversions went into urban and related land uses and one-fourth went into agricultural uses. One-sixth of the diverted timberland was reclassified as reserved timberland and withdrawn from timber harvesting, all within the public ownership category. New areas of water accounted for the remainder of the diversions. Most (95 percent) of the additions to timberland came from nonforest sources, primarily idle pasture and cropland. Timberland decreased throughout the State's peninsula but increased in the panhandle.

- area of publicly owned timberland has increased 16 percent to more than 2.8 million acres, the second highest total of any Southern State. Florida ranks first in the South in the amount of public timberland that is State-owned. Over four-fifths of the increase in public timberland was due to acquisitions by State agencies. Public ownerships now account for 19 percent of Florida's timberland. In contrast, timberland controlled by forest industry decreased 16 percent to 4.6 million acres. Forest industry presently controls 31 percent of Florida's timberland. Timberland under nonindustrial private forest (NIPF) ownerships increased 2 percent to 7.2 million acres. Nonindustrial private forest lands account for 49 percent of Florida's timberland, the lowest proportion of any Southern State. The NIPF category increase resulted solely from gains in the corporate faction, as both the farmer-owned and the other individual groups lost timberland acreage.

Farmers now hold less than 1.0 million acres of timberland, other individuals nearly 3.6 million acres, and other corporate 2.6 million acres.

- area in pine plantations continues to increase, this time by 15 percent to more than 4.6 million acres. Pine plantations now account for almost one-third of Florida's timberland, the highest proportion of any
Southern State. The area in natural pine stands continues to decrease, most recently by 20 percent to 2.8 million acres. Natural pine stands currently represent 19 percent of the State's timberland. Oak-pine stands have increased 22 percent to nearly 1.5 million acres, accounting for 10 percent of all timberland. The remaining area in hardwood stands decreased 8 percent to 5.7 million acres; they comprise 39 percent of Florida's timberland.

- pine forest types are still predominantly slash pine, despite a 1 percent decline to 5.1 million acres. Loblolly pine type increased by 40 percent to 0.8 million acres, superseding longleaf pine type as the second most prevalent pine type. Area of longleaf pine type continues to shrink and is now third in abundance after a 22 percent reduction to 0.7 million acres. Sand pine type increased in area by 4 percent to more than 0.6 million acres.

- area receiving a final harvest and remaining in timberland averaged nearly one-quarter million acres annually. Almost four-fifths of these final harvests took place in pine stands, with pine plantations accounting for 47 percent and natural pine stands 32 percent. Just over one-fifth of the final harvests occurred in hardwood stands, with oak-pine stands accounting for 5 percent, upland hardwood stands nearly 3 percent, and lowland hardwood stands 13 percent. More than one-half of the final harvests occurred on lands controlled by forest industry. Nonindustrial private forest lands accounted for 39 percent of the final harvests, and public lands the remaining 8 percent. In addition to these final harvests, partial harvests and thinnings accounted for an average of 63,000 acres annually. Fire, insects, disease, weather, and other natural agents damaged over 100,000 acres each year.

- an average of 311,000 acres were artificially and naturally regenerated each year. Four-fifths of the regeneration resulted in the establishment of new pine stands, an area 26 percent larger than the pine stand acreage harvested annually. Over three-fourths of the regeneration was accomplished by artificial means. Forest industry lands accounted for 53 percent of the 
artificial regeneration, NIPF lands for 40 percent, and public lands 7 percent. In contrast, NIPF lands accounted for nearly 71 percent of the natural regeneration, forest industry for 21 percent, and public lands 8 percent.

- volume of softwood growing stock increased nearly 5 percent to 9.4 billion cubic feet. Only the 10-, 12-, and 22-inch and larger softwood diameter classes decreased in volume. Volume changes by ownership parallel acreage changes by ownership. Volume of softwood growing stock increased 26 percent on public land to 2.5 billion cubic feet, and 8 percent on NIPF land to nearly 4.3 billion cubic feet. In contrast, inventory of softwood growing stock on forest industry land decreased 14 percent to 2.6 billion cubic feet. Softwood species increasing in volume included slash pine by 10 percent to 4.3 billion cubic feet, loblolly pine by 33 percent to 0.9 billion cubic feet, and sand pine by 17 percent to 490 million cubic feet. Softwood species decreasing in volume included longleaf pine by 10 percent to less than 1.1 billion cubic feet, pond pine by 3 percent to 160 million cubic feet, and the cypresses by 6 percent to 2.3 billion cubic feet. Pine plantations contain 29 percent of the softwood growing stock volume, natural pine stands 33 percent, lowland hardwood stands 27 percent, oak-pine stands 9 percent, and upland hardwood stands 2 percent. Volume of softwood sawtimber increased 6 percent to 28.3 billion board feet.

- volume of hardwood growing stock increased almost 10 percent to more than 5.9 billion cubic feet. All hardwood diameter classes increased in volume except those above 20 inches. By ownership, hardwood volume changes were driven by the major shifts in acreage described earlier. Volume of hardwood growing stock increased 45 percent on public land to 1.3 billion cubic feet and 9 percent on NIPF land to 3.2 billion cubic feet. Hardwood growing stock volume decreased 9 percent to 1.4 billion cubic feet on forest industry land. About 69 percent of the hardwood growing stock volume is located in lowland hardwood stands, nearly 19 percent is in upland hardwood stands, 8 percent in oak-pine stands, and the remainder in pine stands. Collectively, the oaks account for 31 percent of the hardwood inventory, and increased 15 percent to 1.8 billion cubic feet. Tupelo and blackgum increased slightly to 1.5 billion cubic feet. Bay and magnolia species increased 16 percent to less than 0.9 billion cubic feet. Sweetgum increased 11 percent to 563 million cubic feet, and soft maple increased 17 percent to $\mathbf{4 4 5}$ million cubic feet. Ash decreased in volume by 5 percent to 339 million cubic feet. Volume of hardwood sawtimber increased 14 percent to 17.0 billion board feet.
- net annual growth of softwood growing stock increased 9 percent to 532 million cubic feet. Pine plantations provided 62 percent of the softwood growth. Nonindustrial private forest and forest industry lands each accounted for 43 percent of the softwood growth, and public lands contributed 14 percent. Softwood growth increased 24 percent on NIPF land and 1 percent on public land, while decreasing 1 percent on forest industry land. Overall, softwood growth exceeds softwood removals by 13 percent. Although softwood growth exceeds removals in each ownership category, the excess ranges from 36 percent on public land to 15 percent on NIPF land to 4 percent on forest industry land. Net annual growth of softwood sawtimber decreased 5 percent to 1.4 billion board feet. Hardwood net annual growth increased 14 percent to 161 million cubic feet. About 59 percent of the hardwood growth occurred in lowland hardwood stands. Nonindustrial private forest land accounted for 58 percent of the hardwood growth, forest industry 25 percent, and public 17 percent. Hardwood growth increased 14 percent on NIPF land, 4 percent on forest industry land, and 35 percent on public land. Overall, hardwood growth exceeds removals by 83 percent. On NIPF and public lands, hardwood growth is approximately double removals, but the excess is under 50 percent on lands controlled by forest industry. Net annual growth of hardwood sawtimber increased 13 percent to 543 million board feet.

- annual removals of softwood growing stock changed little at 473 million cubic feet. Almost half of the softwood removals came from pine plantations. Forest industry land supplied 46 percent of the State's softwood removals, NIPF land 42 percent, and public land 12 percent. Softwood removals decreased 3 percent on forest industry land and less than 1 percent on NIPF land, but increased 13 percent on public land. The increase on public land occurred primarily on State properties. Two-thirds of the softwood volume removed came from trees 10 inches in diameter and smaller. Removals of softwood sawtimber decreased 4 percent to 1.2 billion board feet. In contrast to softwoods, annual removals of hardwood growing stock increased 33 percent to 88 million cubic feet. Twothirds of the hardwood removals came from lowland hardwood stands. Nonindustrial private forest land provided 54 percent of the hardwood removals, forest industry 31 percent, and 15 percent came from public land. Hardwood removals decreased 14 percent on forest industry land but increased 44 percent on NIPF land and more than tenfold on public land. The increase on public land was primarily confined to State agencies. Over one-half of the hardwood growing stock removals came from trees between 10 and 16 inches in diameter. Removals of hardwood sawtimber increased 48 percent to 265 million board feet. 
- annual mortality of softwood growing stock decreased 14 percent to 54 million cubic feet. Mortality reduced gross growth of softwoods by 9 percent. The leading identifiable causes of mortality to softwoods in descending order were weather, insects, disease, and fire. Nonindustrial private forest and forest industry lands showed decreases in softwood mortality, while public lands showed increases. Most of the increase on public land occurred on State lands. Shifts in ownership patterns as a result of land acquisition by State agencies influenced the recorded increase. Annual mortality of softwood growing stock included 174 million board feet of sawtimber. Annual mortality of hardwood growing stock decreased 13 percent to 52 million cubic feet. Mortality reduced gross growth of hardwoods by 24 percent. Weather, followed by disease and suppression were the leading identifiable causes of mortality in hardwoods. Hardwood mortality decreased on NIPF and forest industry lands while increasing on public lands (primarily State land). Annual mortality of hardwood growing stock included 157 million board feet of sawtimber.

\section{How the Inventory is Made}

Procedures used in the seventh inventory of the forest resources in Florida included seven basic steps.

1. In the Northwest and Northeast units, estimates of forest and nonforest areas were based on the ground classification of 85,107 sample clusters systematically spaced on the latest aerial photographs available. A subsample of 6,513 of the 16-point clusters was ground checked, and a linear regression was fitted to the data to develop the relationship between the photo and ground classification of the subsample. This procedure provides a means for adjusting the initial estimates of area for change in land use since date of photography and for photo misclassification. In the Central and South units, estimates of forest and nonforest areas were based on the ground classification of 6,030 sample clusters systematically distributed across the landscape. At each of the sample clusters, 16 points were classified as to land use.

2. Estimates of timber volume and forest classification were based on measurements recorded at 5,424 ground sample locations systematically distributed on timberland. The plot design at each location was based on a cluster of 10 points. In most cases, variable plots, established by using a basal-area factor of 37.5 square feet per acre, were systematically spaced within a single forest condition at 5 of the 10 cluster points. Trees less than 5 inches d.b.h. were tallied on a fixed-radius plot around each point center.
3. Equations prepared from detailed measurements collected on standing trees in Florida, and similar measurements taken throughout the Southeast, were used to compute the volume of individual tally trees. A mirror caliper and sectional aluminum poles were used to obtain the additional measurements required to construct volume equations. Forest biomass estimates were made from equations developed by the Utilization of Southern Timber Research Work Unit, Southern Research Station, Athens, GA.

4. Felled trees were measured at 50 active cutting operations. These data will supplement the standingtree volume data and be used to generate utilization factors for product and species groups.

5. Estimates of growth, removals, and mortality were determined from the remeasurement of 5,591 permanent sample plots established in the sixth survey.

6. Ownership information was collected from correspondence, public records, and local contacts. In counties where the sample missed a particular ownership class, temporary sample plots were added.

7. All field data were sent to Asheville for editing and were entered into disk and magnetic-tape storage for processing. Final estimates were based on statistical summaries of the data. 


\section{Statistical Reliability}

FIA inventories employ sampling methods designed to achieve reliable statistics at the Survey Unit and State levels. A measure of reliability of inventory statistics is provided by sampling errors. These sampling errors mean that the chances are two out of three that the true population value is within the limits indicated by a confidence interval. Sampling errors (in percent) and associated confidence intervals around the sample estimates for timberland area, inventory volumes, and components of change are presented in the following table.

Item

Sample estimate Sampling and error confidence interval (percent)

\begin{tabular}{lrll} 
Timberland (1,000 acres) & $14,650.7 \pm 58.6$ & 0.40 \\
$\begin{array}{l}\text { Growing stock }\left(M t^{3}\right) \\
\text { Inventory }\end{array}$ & $15,366.4 \pm 253.5$ & 1.65 \\
Net annual growth & $692.6 \pm 11.9$ & 1.72 \\
Annual removals & $560.7 \pm 20.1$ & 3.59 \\
Annual mortality & $105.7 \pm 4.8$ & 4.55 \\
& & & \\
Sawtimber $(M$ fbm) & & & \\
Inventory & $45,287.2 \pm 969.1$ & 2.14 \\
Net annual growth & $1,965.2 \pm 43.2$ & 2.20 \\
Annual removals & $1,482.9 \pm 64.8$ & 4.37 \\
Annual mortality & $331.2 \pm 20.1$ & 6.06 \\
\hline
\end{tabular}

Sampling error increases as the area or volume considered decreases in magnitude. Sampling errors and associated confidence intervals are often unacceptably high for small components of the total resource. Statistical confidence may be computed for any subdivision of Survey Unit or State totals using the following formula. Sampling errors obtained from this method are only approximations of reliability because this process assumes constant variance across all subdivisions of totals.

$$
S E_{s}=S E_{t} \frac{\sqrt{x_{t}}}{\sqrt{x_{s}}},
$$

where

$$
\begin{aligned}
& \mathrm{SE}_{s}=\begin{array}{l}
\text { sampling error for subdivision of Survey } \\
\text { Unit or State total, }
\end{array} \\
& \mathrm{SE}_{t}=\text { sampling error for Survey Unit or State total, } \\
& \mathrm{X}_{s}=\begin{array}{l}
\text { sum of values for the variable of interest } \\
\text { (area or volume) for subdivision of Survey } \\
\text { Unit or State, }
\end{array} \\
& \mathrm{X}_{t}=\text { total area or volume for Survey Unit or State. }
\end{aligned}
$$

For example, the estimate of sampling error for growing-stock volume on other private timberland is computed as:

$$
S E_{S}=1.65 \frac{\sqrt{15,366.4}}{\sqrt{7,477.4}}=2.37
$$

Thus, the sampling error is 2.37 percent, and the resulting confidence interval (two times out of three) for growing-stock inventory on other private timberland is $7,477.4 \pm 177.2$ million cubic feet.

County statistics are provided, but users are cautioned that the accuracy of individual county data is highly variable. Individual county statistics are provided so any combination of counties may be added together until the totals are large enough to meet the desired degree of reliability. Sampling errors for key resource items for individual counties are provided in the following table. 
Sampling errors for county and unit totals, in terms of one standard error, Florida, 1995

\begin{tabular}{|c|c|c|c|c|c|c|c|c|c|}
\hline \multirow[b]{2}{*}{ County } & \multirow{2}{*}{$\begin{array}{c}\text { Timberland } \\
\text { area }\end{array}$} & \multicolumn{3}{|c|}{$\begin{array}{l}\text { Cubic-foot volume } \\
\text { of growing stock }\end{array}$} & \multirow[b]{2}{*}{ County } & \multirow{2}{*}{$\begin{array}{c}\text { imberland } \\
\text { area }\end{array}$} & \multicolumn{3}{|c|}{$\begin{array}{l}\text { Cubic-foot volume } \\
\text { of growing stock }\end{array}$} \\
\hline & & Inventory & Growth & Removals & & & Inventory & Growth & Removals \\
\hline & \multicolumn{4}{|c|}{ Sampling error ${ }^{a}$} & & \multicolumn{4}{|c|}{ Sampling error ${ }^{a}$} \\
\hline Alachua & 2.15 & 10.67 & 9.24 & 21.30 & Lee & 15.41 & 35.79 & 46.89 & 72.91 \\
\hline Baker & 0.78 & 10.38 & 7.97 & 21.97 & Leon & 1.73 & 8.74 & 10.56 & 27.09 \\
\hline Bay & 0.93 & 13.77 & 10.36 & 16.14 & Levy & 1.49 & 8.24 & 8.57 & 18.96 \\
\hline Bradford & 1.68 & 16.32 & 13.83 & 40.41 & Liberty & 0.35 & 7.67 & 10.24 & 22.41 \\
\hline Brevard & 11.65 & 25.73 & 25.62 & 52.59 & Madison & 1.98 & 13.53 & 12.08 & 20.58 \\
\hline Broward & 0.00 & 0.00 & 0.00 & 0.00 & Manatee & 13.51 & 30.44 & 31.14 & 85.10 \\
\hline Calhoun & 1.27 & 11.38 & 11.59 & 22.31 & Marion & 1.40 & 8.12 & 7.97 & 18.24 \\
\hline Charlotte & 22.20 & 40.62 & 58.14 & 103.71 & Martin & 19.19 & 32.02 & 49.41 & 0.00 \\
\hline Citrus & 5.89 & 16.55 & 16.99 & 29.41 & Monroe & 0.00 & 0.00 & 0.00 & 0.00 \\
\hline Clay & 2.36 & 10.92 & 10.33 & 24.59 & Nassau & 0.97 & 10.17 & 8.72 & 20.12 \\
\hline Collier & 3.05 & 14.78 & 42.63 & 44.92 & Okaloosa & 1.48 & 8.59 & 9.41 & 27.97 \\
\hline Columbia & 1.31 & 9.17 & 9.17 & 21.02 & Okeechobee & 15.03 & 26.45 & 25.11 & 74.50 \\
\hline Dade & 0.00 & 0.00 & 0.00 & 0.00 & Orange & 8.28 & 17.25 & 17.27 & 38.59 \\
\hline De Soto & 16.18 & 32.08 & 33.11 & 58.33 & Osceola & 7.96 & 15.05 & 20.41 & 68.20 \\
\hline Dixie & 0.68 & 8.08 & 7.27 & 17.54 & Palm Beach & 0.00 & 0.00 & 0.00 & 0.00 \\
\hline Duval & 2.71 & 10.56 & 10.08 & 32.48 & Pasco & 7.65 & 18.17 & 32.33 & 32.64 \\
\hline Escambia & 1.94 & 10.12 & 9.48 & 23.70 & Pinellas & 48.86 & 111.25 & 110.69 & 129.56 \\
\hline Flagler & 1.21 & 11.96 & 9.09 & 21.21 & Polk & 6.88 & 14.68 & 13.86 & 35.01 \\
\hline Franklin & 0.76 & 11.51 & 11.01 & 32.35 & Putnam & 1.66 & 12.24 & 12.83 & 23.46 \\
\hline Gadsden & 1.63 & 11.81 & 10.30 & 31.06 & Santa Rosa & 0.99 & 8.30 & 7.81 & 17.20 \\
\hline Gilchrist & 3.25 & 17.24 & 17.69 & 37.24 & Sarasota & 14.10 & 26.91 & 28.46 & 41.76 \\
\hline Glades & 12.65 & 37.68 & 30.44 & 38.08 & Seminole & 10.59 & 21.51 & 27.38 & 74.32 \\
\hline Gulf & 1.61 & 17.17 & 15.60 & 26.82 & St. Johns & 1.96 & 10.98 & 10.81 & 21.48 \\
\hline Hamilton & 2.32 & 11.72 & 11.77 & 24.32 & St. Lucie & 21.31 & 39.04 & 32.71 & 51.72 \\
\hline Hardee & 12.07 & 22.24 & 22.28 & 100.73 & Sumter & 7.78 & 14.39 & 19.92 & 46.16 \\
\hline Hendry & 14.60 & 34.83 & 37.72 & 46.06 & Suwannee & 3.02 & 11.93 & 11.66 & 32.91 \\
\hline Hernando & 6.73 & 14.83 & 20.67 & 43.66 & Taylor & 0.68 & 10.18 & 7.77 & 13.05 \\
\hline Highlands & 12.60 & 26.02 & 35.64 & 77.18 & Union & 2.33 & 16.37 & 14.54 & 39.66 \\
\hline Hillsborough & 10.78 & 20.78 & 21.70 & 59.70 & Volusia & 1.45 & 8.51 & 15.51 & 22.93 \\
\hline Holmes & 2.60 & 15.08 & 14.91 & 35.18 & Wakulla & 1.04 & 10.68 & 14.21 & 27.32 \\
\hline Indian River & 22.59 & 46.14 & 121.74 & 103.76 & Walton & 1.31 & 8.60 & 9.12 & 22.35 \\
\hline Jackson & 2.11 & 12.90 & 11.47 & 17.91 & Washington & 1.43 & 11.87 & 11.16 & 30.51 \\
\hline Jefferson & 1.42 & 10.47 & 11.21 & 23.85 & & & & & \\
\hline Lafayette & 1.31 & 12.77 & 11.12 & 18.61 & Total & 0.40 & 1.65 & 1.72 & 3.59 \\
\hline Lake & 5.94 & 13.03 & 20.98 & 40.23 & & & & & \\
\hline
\end{tabular}




\section{Definitions}

Basal area. The area in square feet of the cross section at breast height of a single tree or of all the trees in a stand, usually expressed in square feet per acre.

Biomass. The aboveground green weight of solid wood and bark in live trees 1.0-inch d.b.h. and larger from the ground to the tip of the tree. All foliage is excluded. The weight of wood and bark in lateral limbs, secondary limbs, and twigs under 0.5 inch in diameter at the point of occurrence on sapling-size trees is included but is excluded on poletimber and sawtimber-size trees.

Bole. That portion of a tree between a 1-foot stump and a 4-inch top diameter outside bark (d.o.b.) in trees 5.0 inches d.b.h. and larger.

Broad management class. A classification of timberland based on forest type and stand origin.

Pine plantation. Stands that have been artificially regenerated by planting or direct seeding and with a southern yellow pine, white pine-hemlock, or other softwood forest type.

Natural pine. Stands that have not been artificially regenerated and with a southern yellow pine, white pine-hemlock, or other softwood forest type.

Oak-pine. Stands with a forest type of oak-pine.

Upland hardwood. Stands with a forest type of oak-hickory, chestnut oak, southern scrub oak, or maple-beech-birch.

Lowland hardwood. Stands with a forest type of oak-gum-cypress, elm-ash-cottonwood, palm, or other tropical.

Census water. Streams, sloughs, estuaries, canals, and other moving bodies of water 200 feet wide and greater, and lakes, reservoirs, ponds, and other permanent bodies of water 4.5 acres in area and greater.

Commercial forest land. (see: Timberland).

Commercial species. Tree species currently or potentially suitable for industrial wood products. Noncommercial species are excluded.
Cropland. Land under cultivation within the past 24 months, including orchards and land in soil-improving crops but excluding land cultivated in developing improved pasture. Also includes idle farmland.

D.b.h. Tree diameter in inches (outside bark) at breast height (4.5 feet above the ground).

Diameter class. A classification of trees based on tree d.b.h. Two-inch diameter classes are commonly used by Forest Inventory and Analysis, with the even inch as the approximate midpoint for a class. For example, the 6-inch class includes trees 5.0-6.9 inches d.b.h.

Farm. Land on which agricultural operations are being conducted and sale of agricultural products totaled $\$ 1,000$ or more during the year.

Farm operator. A person who operates a farm, either doing the work or directly supervising the work.

Farmer-owned land. (see: Other private land).

Forest industry land. Land owned by companies or individuals operating primary wood-using plants.

Forest industry-leased land. Land leased or under management contracts to forest industry from other owners for periods of one forest rotation or longer. Land under cutting contracts is not included.

Forest land. Land at least 16.7 percent stocked by forest trees of any size, or formerly having had such tree cover, and not currently developed for nonforest use.

Forest type. A classification of forest land based on the species forming a plurality of live-tree stocking.

White pine-hemlock. Forests in which eastern white pine, red pine, or jack pine, singly or in combination, constitute a plurality of the stocking. (Common associates include hemlock, birch, and maple.)

Spruce-fir. Forests in which spruce or true firs, singly or in combination, constitute a plurality of the stocking. (Common associates include maple, birch, and hemlock.)

Longleaf-slash pine. Forests in which longleaf or slash pine, singly or in combination, constitute a plurality of the stocking. (Common associates include oak, hickory, and gum.) 
Loblolly-shortleaf pine. Forests in which loblolly pine, shortleaf pine, or other southern yellow pines, except longleaf or slash pine, singly or in combination, constitute a plurality of the stocking. (Common associates include oak, hickory, and gum.)

Oak-pine. Forests in which hardwoods (usually upland oaks) constitute a plurality of the stocking but in which pines account for 25 to 50 percent of the stocking. (Common associates include gum, hickory, and yellow-poplar.)

Oak-hickory. Forests in which upland oaks or hickory, singly or in combination, constitute a plurality of the stocking, except where pines account for 5 to 50 percent, in which case the stand would be classified oak-pine. (Common associates include yellow-poplar, elm, maple, and black walnut.)

Oak-gum-cypress. Bottom-land forests in which tupelo, blackgum, sweetgum, oaks, or southern cypress, singly or in combination, constitue a plurality of the stocking, except where pines account for 25 to 50 percent, in which case the stand would be classified oak-pine. (Common associates include cottonwood, willow, ash, elm, hackberry, and maple.)

Elm-ash-cottonwood. Forests in which elm, ash, or cottonwood, singly or in combination, constitute a plurality of the stocking. (Common associates include willow, sycamore, beech, and maple.)

Maple-beech-birch. Forests in which maple, beech, or yellow birch, singly or in combination, constitute a plurality of the stocking. (Common associates include hemlock, elm, basswood, and white pine.)

Palm, other tropicals. Forests in which palms and other tropicals constitute a plurality of the stocking.

Gross growth. Annual increase in merchantable volume of trees in the absence of cutting and mortality. (Gross growth includes survivor growth, ingrowth, growth on ingrowth, growth on removals prior to removal, and growth on mortality prior to death.)

Growing-stock trees. Live sawtimber-size trees of commercial species containing at least a 12-foot log, or two noncontiguous saw logs each 8 feet or longer, meeting minimum grade requirements (hardwoods must qualify as a log grade of either 3 or 4 ; softwoods must qualify as a log grade 3 ) with at least one-third of the gross board-foot volume (International 1/4-inch rule) between a 1 -foot stump and the minimum sawlog top being sound, or a live tree below sawtimber size that will prospectively qualify under the above standards.

Growing-stock volume. Volume (cubic feet) of solid wood in growing-stock trees 5.0 inches d.b.h. and larger, from a 1 -foot stump to a minimum 4.0-inch top diameter, outside bark, on the central stem. Volume of solid wood in primary forks from the point of occurrence to a minimum 4.0-inch top diameter outside bark is included.

Hardwoods. Angiosperms; dicotyledonous trees (including all palm species which are monocotyledonous), usually broadleaf and deciduous.

Soft hardwoods. Soft-textured hardwoods such as boxelder, red and silver maples, hackberry, loblollybay, sweetgum, yellow-poplar, magnolia, sweetbay, water tupelo, blackgum, sycamore, cottonwood, black cherry, willow, basswood, and elm.

Hard hardwoods. Hard-textured hardwoods such as sugar maple, birch, hickory, dogwood, persimmon (forest grown), black locust, beech, ash, honey-locust, holly, black walnut, mulberry, and all commercial oaks.

Idle farmland. Cropland, orchard, improved pasture, and farm sites not tended within the past 2 years, and currently less than 16.7 percent stocked with live trees.

Improved pasture. Land currently improved for grazing by cultivation, seeding, irrigation, or clearing of trees or brush.

Industrial wood. All roundwood products except fuelwood.

Ingrowth. The number or net volume of trees that grow large enough during a specified year to qualify as saplings, poletimber, or sawtimber.

Land area. The area of dry land and land temporarily or partly covered by water such as marshes, swamps, and river floodplains (omitting tidal flats below mean high tide), streams, sloughs, estuaries, and canals less than 200 feet wide, and lakes, reservoirs, and ponds less than 4.5 acres in area.

Live trees. All trees 1.0 inch d.b.h. and larger which are not dead at the time of inventory. 
Live-tree volume. Volume (cubic feet) of wood above the ground line in live trees 1.0-inch d.b.h. and larger. The volume in twigs and lateral limbs smaller than 0.5 inch in diameter at the point of occurrence on sapling-size trees is included but is excluded on poletimber- and sawtimber-size trees.

Log grade. A classification of logs based on external characteristics as indicators of quality or value.

Logging residues. The unused merchantable portion of growing-stock trees cut or destroyed during logging operations.

Manageable stand. Timberland at least 60 percent stocked with growing-stock trees that can be featured together under a management scheme.

Merchantable portion. That portion of live trees 5.0 inches d.b.h. and larger between a 1-foot stump and a minimum 4.0-inch top diameter outside bark on the central stem. That portion of primary forks from the point of occurrence to a minimum 4.0-inch top diameter outside bark is included.

Merchantable volume. Solid-wood volume in merchantable portion of live trees.

Miscellaneous Federal land. Federal land other than national forests, land administered by the Bureau of Land Management, and land administered by the Bureau of Indian Affairs.

Miscellaneous private land. (see: Other private land).

Mortality. The merchantable volume in trees that have died from natural causes during a specified period.

National forest land. Federal land that has been legally designated as national forests or purchase units, and other land under the administration of the Forest Service, including experimental areas and BankheadJones Title III land.

Net annual growth. The net change in merchantable volume for a specific year in the absence of cutting (gross growth minus mortality for that specified year).

Net volume. Gross volume of wood less deductions for rot, sweep, or other defect affecting use for timber products.

Noncommercial species. Tree species of typically small size, poor form, or inferior quality which normally do not develop into trees suitable for industrial wood products.
Nonforest land. Land that has never supported forests and land formerly forested where timber production is precluded by development for other uses.

Nonindustrial private forest (NIPF) land. (see: Other private land).

Nonstocked forest land. Timberland less than 16.7 percent stocked with growing-stock trees.

Other private land. Privately owned land excluding forest industry land or forest industry-leased land. Also referred to as nonindustrial private forest (NIPF) land.

Farmer-owned land. Owned by farm operators, excluding incorporated farm ownerships.

Other individual land. Owned by individuals other than farm operators.

Other corporate land. Owned by corporations, including incorporated farm ownerships.

Other removals. The growing-stock volume of trees removed from the inventory by cultural operations such as timber stand improvement, land clearing, and other changes in land use that result in the removal of the trees from timberland.

Plant residues. Wood material generated in the production of timber products at primary manufacturing plants.

Coarse residues. Material, such as slabs, edgings, trim, veneer cores and ends, which is suitable for chipping.

Fine residues. Material, such as sawdust, shavings, and veneer chippings, which is not suitable for chipping.

Plant byproducts. Residues (coarse or fine) utilized in the further manufacture of industrial products or for consumer use, or utilized as fuel.

Unused plant residues. Residues (coarse or fine) that are not used for any product, including fuel.

Poletimber-size trees. Live trees at least 5.0 inches d.b.h. but smaller than sawtimber size.

Primary wood-using plants. Industries that receive roundwood or chips from roundwood for the manufacture of products such as veneer, pulp, and lumber.

Productive-reserved forest land. (see: Reserved timberland). 
Rangeland. Land on which the natural vegetation is predominantly native grasses, grasslike plants, forbs, or shrubs valuable for forage, not qualifying as timberland and not developed for another land use. Rangeland includes natural grassland and savannah.

Reserved timberland. Forest land sufficiently productive to qualify as timberland, but withdrawn from timber utilization through statute or administrative designation.

Rotten trees. Live trees of commercial species that do not contain at least one 12-foot saw log, or two noncontiguous saw logs, each 8 feet or longer, now or prospectively, primarily because of rot or missing sections, and with less than one-third of the gross board-foot tree volume in sound material.

Rough trees. Live trees of commercial species that do not contain at least one 12-foot saw log, or two noncontiguous saw logs, each 8 feet or longer, now or prospectively, primarily because of roughness, poor form, splits, and cracks, and with less than one-third of the gross board-foot tree volume in sound material; and live trees of noncommercial species.

Roundwood (roundwood logs). Logs, bolts, or other round sections cut from trees for industrial or consumer uses.

Roundwood chipped. Any timber cut primarily for pulpwood, delivered to nonpulpmills, chipped, and then sold to pulpmills as residues, including chipped tops, jump sections, whole trees, and pulpwood sticks.

Roundwood products. Any primary product such as lumber, poles, pilings, pulp, or fuelwood which is produced from roundwood.

Salvable dead trees. Standing or down dead trees considered utilizable by Forest Inventory and Analysis standards.

Saplings. Live trees 1.0 to 5.0 inches d.b.h.

Saw log. A log meeting minimum standards of diameter, length, and defect, including logs at least 8 feet long, sound and straight, and with a minimum diameter inside bark for softwoods of 6 inches $(8$ inches for hardwoods).

Saw-log portion. That part of the bole of sawtimber trees between a 1 -foot stump and the saw-log top, including the portion of forks large enough to contain a saw log.
Saw-log top. The point on the bole of sawtimber trees above which a conventional saw log cannot be produced. The minimum saw-log top is 7.0 inches in diameter outside bark (d.o.b.) for softwoods and 9.0 inches d.o.b. for hardwoods.

Sawtimber-size trees. Softwoods 9.0 inches d.b.h. and larger and hardwoods 11.0 inches d.b.h. and larger.

Sawtimber volume. Growing-stock volume in the sawlog portion of sawtimber-size trees in board feet (International $1 / 4$-inch rule).

Seedlings. Trees less than 1.0 inch in d.b.h. Only seedlings of a commercial species that are not overtopped and are more than 6 inches tall for softwoods and 1 foot tall for hardwoods are counted.

Site class. A classification of forest land in terms of inherent capacity to grow crops of industrial wood based on fully stocked natural stands, by annual production capacity.

Softwoods. Gymnosperms; in the order Coniferales, usually evergreen (includes the genus Taxodium which is deciduous), having needles or scalelike leaves.

Pines. Yellow pine species which include loblolly, longleaf, slash, pond, shortleaf, pitch, Virginia, sand, spruce, and Table Mountain pines.

Other softwoods. Cypress, eastern red cedar, white cedar, eastern white pine, eastern hemlock, spruce, and fir.

Stand-size class. A classification of forest land based on the diameter class distribution of live trees in the stand.

Sawtimber stands. Stands at least 16.7 percent stocked with live trees, with half or more of total stocking in sawtimber and poletimber trees, and with sawtimber stocking at least equal to poletimber stocking.

Poletimber stands. Stands at least 16.7 percent stocked with live trees, of which half or more of total stocking is in poletimber and sawtimber trees, and with poletimber stocking exceeding that of sawtimber.

Sapling-seedling stands. Stands at least 16.7 percent stocked with live trees of which more than half of total stocking is saplings and seedlings. 
State, county, and municipal land. Land owned by States, counties, and local public agencies or municipalities, or land leased to these governmental units for 50 years or more.

Stocking. The degree of occupancy of land by trees, measured by basal area or the number of trees in a stand and spacing in the stand, compared with a minimum standard, depending on tree size, required to fully utilize the growth potential of the land.

Fully stocked. 100 percent or more stocking.

Medium stocked. 60 to 99 percent stocking.

Poorly stocked. Less than 60 percent stocking.

Density of trees and basal area per acre required for full stocking

\begin{tabular}{ccc}
\hline $\begin{array}{l}\text { D.b.h. } \\
\text { class }\end{array}$ & $\begin{array}{c}\text { Trees per acre } \\
\text { for full stocking }\end{array}$ & $\begin{array}{c}\text { Basal area } \\
\text { per acre }\end{array}$ \\
\hline Seedlings & 600 & - \\
2 & 560 & - \\
4 & 460 & - \\
6 & 340 & 67 \\
8 & 240 & 84 \\
10 & 155 & 85 \\
12 & 115 & 90 \\
14 & 90 & 96 \\
16 & 72 & 101 \\
18 & 60 & 106 \\
20 & 51 & 111 \\
\hline
\end{tabular}

Survivor growth. The merchantable volume increment on trees 5.0 inches d.b.h. and larger in the inventory at the beginning of the year and surviving to its end.

Timberland. Forest land that is capable of producing 20 cubic feet of industrial wood per acre per year and not withdrawn from timber utilization.
Timber products. Roundwood products and byproducts.

Timber removals. The merchantable volume of trees removed from the inventory by harvesting, cultural operations such as stand improvement, land clearing, or changes in land use.

Top. The portion of the main stem and forks from a 4.0-inch diameter outside bark to the tips of the main stem and forks, plus all other limbs above the 4.0-inch top at least 0.5 inch in diameter at their point of occurrence.

Treatment opportunity. A classification of the management or treatment that would most improve for timber production the existing condition of the stand being sampled.

Tree. Woody plants having one erect perennial stem or trunk at least 3 inches d.b.h., a more or less definitely formed crown of foliage, and a height of at least 13 feet.

Tree grade. A classification of sawtimber trees based on the log grade of the butt log in the tree.

Unproductive forest land. (see: Woodland).

Upper-stem portion. That part of the main stem or fork of sawtimber trees above the saw-log top to minimum top diameter 4.0 inches outside bark or to the point where the main stem or fork breaks into limbs.

Urban and other areas. Areas developed for residential, industrial, or recreational purposes, school yards, cemeteries, roads, railroads, airports, beaches, powerlines and other rights-of-way, or other nonforest land not included in any other specified land use class.

Woodland. Forest land incapable of producing 20 cubic feet per acre per year of industrial wood under natural conditions, because of adverse site conditions. 


\section{CONVERSION FACTORS}

Cubic feet of wood per average cord

(excluding bark)

\begin{tabular}{ccccc}
\hline $\begin{array}{l}\text { D.b.h. } \\
\text { class }\end{array}$ & $\begin{array}{c}\text { All } \\
\text { species }\end{array}$ & Pine & $\begin{array}{c}\text { Other } \\
\text { softwood }\end{array}$ & Hardwood \\
\hline 6 & 61.4 & 61.0 & 68.2 & 60.0 \\
8 & 69.3 & 68.1 & 76.0 & 68.4 \\
10 & 74.5 & 73.1 & 81.4 & 73.4 \\
12 & 77.9 & 76.7 & 85.2 & 76.4 \\
14 & 80.3 & 79.4 & 88.2 & 78.4 \\
16 & 81.9 & 81.6 & 90.4 & 79.8 \\
18 & 83.0 & 83.3 & 92.3 & 80.8 \\
20 & 83.8 & 84.8 & 93.8 & 81.5 \\
22 & 84.4 & 86.0 & 95.1 & 82.1 \\
$24+$ & 84.8 & 87.7 & 98.0 & 83.2 \\
\cline { 2 - 5 } Average & 74.5 & 72.0 & 82.5 & 74.5 \\
\hline
\end{tabular}

Metric equivalents of units used in this report

1 acre $=4,046.86$ square meters or 0.404686 hectare

1 cubic foot $=0.028317$ cubic meter

1 inch $=2.54$ centimeters or 0.0254 meter

Breast height $(4.5$ feet $)=1.4$ meters above ground level

1 square foot $=929.03$ square centimeters or 0.0929 square meter

1 square foot per acre basal area $=0.229568$ square meter per hectare

1 pound $=0.454$ kilogram

1 ton $=0.907$ metric ton 


\section{Index of Tables ${ }^{a}$}

\section{County Tables}

1. Area, by county and land class

2. Area of timberland, by county and ownership class

3. Area of timberland, by county and forest-type group

4. Area of timberland, by county and stand-size class

5. Area of timberland, by county and site class

6. Area of timberland, by county and stocking class of growing-stock trees

7. Volume of growing stock and sawtimber on timberland, by county and species group

8. Average net annual growth of growing stock and sawtimber on timberland, by county and species group

9. Average annual removals of growing stock and sawtimber on timberland, by county and species group

\section{State Tables}

10. Area of timberland, by forest type and ownership class

11. Area of timberland, by ownership and stocking classes of growing-stock trees

12. Area of timberland, by forest type and stand-size class

13. Area of timberland, by stand-age and broad management classes, all ownerships

14. Area of timberland, by stand-age and broad management classes, public ownerships

15. Area of timberland, by stand-age and broad management classes, forest industry

16. Area of timberland, by stand-age and broad management classes, other private ownerships

17. Area of timberland, by broad management and stand-volume classes

18. Volume of growing stock on timberland, by broad management class, species group, and stand-age class

19. Average net annual growth of growing stock on timberland, by broad management class, species group, and stand-age class

20. Average annual removals of growing stock on timberland, by broad management class, species group, and stand-age class

21. Merchantable volume of live trees and growing stock on timberland, by forest-type and species groups

22. Area of timberland treated or disturbed annually and retained in timberland, by treatment or disturbance and ownership class

23. Area of timberland treated or disturbed annually and retained in timberland, by treatment or disturbance and broad management class

24. Area of timberland regenerated annually, by type of regeneration and broad management class

25. Area of timberland, by treatment opportunity and broad management classes

26. Area of timberland, by treatment opportunity and ownership classes

27. Merchantable volume of live trees and growing stock on timberland, by ownership class and species group

28. Volume of sawtimber on timberland, by ownership class and species group

29. Average net annual growth and removals of growing stock on timberland, by ownership class and species group 
30. Average net annual growth and removals of sawtimber on timberland, by ownership class and species group

31. Volume of timber on timberland, by class of timber and species group

32. Number of live trees on timberland, by species and diameter class

33. Number of growing-stock trees on timberland, by species and diameter class

34. Merchantable volume of live trees on timberland, by species and diameter class

35. Volume of growing stock on timberland, by species and diameter class

36. Volume of sawtimber on timberland, by species and diameter class

37. Volume of sawtimber on timberland, by species, size class, and tree grade

38. Cubic volume in the merchantable saw-log portion of sawtimber trees on timberland, by species and diameter class
39. Total volume of live trees on timberland, by species and diameter class

40. Green weight of forest biomass on timberland, by species and diameter class

41. Average net annual growth and removals of live timber and growing stock on timberland, by species

42. Average net annual growth and removals of sawtimber on timberland, by species

43. Average annual removals of growing stock on timberland, by species and diameter class

44. Average annual mortality of live timber, growing stock, and sawtimber on timberland, by species

45. Change in number of live trees on timberland, by species group, survey completion date, and diameter class

46. Land area, by land use class, major forest type, and survey completion date

47. Volume of sawtimber, growing stock, and live timber on timberland, by species group, survey completion date, and diameter class

a Tables $1-12,27,29-33,35-38,41,42$, and 44 are common to all Forest Inventory and Analysis forest resource statistical reports of the Eastern United States. 
Table 1-Area, by county and land class, Florida, 1995

\begin{tabular}{|c|c|c|c|c|c|c|}
\hline \multirow[b]{2}{*}{ County } & \multirow[b]{2}{*}{$\begin{array}{c}\text { All } \\
\text { land }^{a}\end{array}$} & \multicolumn{4}{|c|}{ Forest land } & \multirow[b]{2}{*}{$\begin{array}{c}\text { Nonforest } \\
\text { land }^{b}\end{array}$} \\
\hline & & Total & Timberland & Woodland & $\begin{array}{c}\text { Reserved } \\
\text { timberland }\end{array}$ & \\
\hline & \multicolumn{6}{|c|}{ Acres } \\
\hline Alachua & 559,552 & 293,200 & 277,455 & - & 15,745 & 266,352 \\
\hline Baker & 374,560 & 341,420 & 326,523 & - & 14,897 & 33,140 \\
\hline Bay & 488,794 & 399,595 & 396,365 & 2,030 & 1,200 & 89,199 \\
\hline Bradford & 187,616 & 135,197 & 135,197 & - & - & 52,419 \\
\hline Brevard & 651,834 & 99,954 & 93,094 & 6,390 & 470 & 551,880 \\
\hline Broward & 773,689 & 21,101 & - & 21,101 & - & 752,588 \\
\hline Calhoun & 363,130 & 302,590 & 302,590 & - & - & 60,540 \\
\hline Charlotte & 443,949 & 50,668 & 30,289 & 20,379 & - & 393,281 \\
\hline Citrus & 373,504 & 194,786 & 185,932 & 5,146 & 3,708 & 178,718 \\
\hline Clay & 384,730 & 289,846 & 288,246 & - & 1,600 & 94,884 \\
\hline Collier & $1,296,288$ & 602,655 & 190,458 & 263,870 & 148,327 & 693,633 \\
\hline Columbia & 510,182 & 367,717 & 357,483 & 1,096 & 9,138 & 142,465 \\
\hline Dade & $1,244,454$ & 160,640 & - & 127,764 & 32,876 & $1,083,814$ \\
\hline De Soto & 407,891 & 47,759 & 47,759 & - & - & 360,132 \\
\hline Dixie & 450,598 & 387,066 & 385,712 & - & 1,354 & 63,532 \\
\hline Duval & 495,264 & 232,570 & 229,614 & 1,359 & 1,597 & 262,694 \\
\hline Escambia & 424,730 & 257,018 & 250,789 & 4,357 & 1,872 & 167,712 \\
\hline Flagler & 310,413 & 241,116 & 237,592 & 1,345 & 2,179 & 69,297 \\
\hline Franklin & 341,747 & 302,064 & 301,493 & 300 & 271 & 39,683 \\
\hline Gadsden & 330,342 & 253,880 & 253,400 & - & 480 & 76,462 \\
\hline Gilchrist & 223,270 & 134,671 & 134,671 & - & - & 88,599 \\
\hline Glades & 495,040 & 103,368 & 91,648 & 11,720 & - & 391,672 \\
\hline Gulf & 361,638 & 312,153 & 310,232 & 1,145 & 776 & 49,485 \\
\hline Hamilton & 329,523 & 242,409 & 240,094 & - & 2,315 & 87,114 \\
\hline Hardee & 407,910 & 79,530 & 79,530 & - & - & 328,380 \\
\hline Hendry & 737,728 & 71,626 & 62,831 & 8,795 & - & 666,102 \\
\hline Hernando & 306,112 & 158,362 & 155,853 & 2,349 & 160 & 147,750 \\
\hline Highlands & 658,227 & 85,563 & 78,759 & 2,835 & 3,969 & 572,664 \\
\hline Hillsborough & 672,653 & 122,579 & 96,466 & 12,297 & 13,816 & 550,074 \\
\hline Holmes & 308,832 & 211,102 & 210,742 & - & 360 & 97,730 \\
\hline Indian River & 322,080 & 31,185 & 22,839 & 7,146 & 1,200 & 290,895 \\
\hline Jackson & 586,099 & 327,146 & 325,426 & - & 1,720 & 258,953 \\
\hline Jefferson & 382,586 & 286,499 & 284,399 & - & 2,100 & 96,087 \\
\hline Lafayette & 347,411 & 297,321 & 297,321 & - & - & 50,090 \\
\hline Lake & 609,990 & 269,412 & 248,490 & 5,698 & 15,224 & 340,578 \\
\hline Lee & 514,304 & 118,714 & 60,872 & 57,842 & - & 395,590 \\
\hline Leon & 426,752 & 306,075 & 305,389 & - & 686 & 120,677 \\
\hline Levy & 715,802 & 476,562 & 462,794 & 895 & 12,873 & 239,240 \\
\hline Liberty & 534,995 & 513,260 & 503,233 & - & 10,027 & 21,735 \\
\hline Madison & 442,854 & 326,403 & 326,368 & - & 35 & 116,451 \\
\hline Manatee & 474,368 & 52,479 & 40,003 & 5,666 & 6,810 & 421,889 \\
\hline Marion & $1,010,554$ & 588,149 & 568,275 & 302 & 19,572 & 422,405 \\
\hline Martin & 355,629 & 51,434 & 40,647 & 7,407 & 3,380 & 304,195 \\
\hline Monroe & 638,253 & 364,908 & - & 275,857 & 89,051 & 273,345 \\
\hline Nassau & 417,037 & 324,825 & 323,374 & 512 & 939 & 92,212 \\
\hline Okaloosa & 598,906 & 449,230 & 448,276 & 597 & 357 & 149,676 \\
\hline Okeechobee & 495,571 & 45,417 & 43,331 & 1,586 & 500 & 450,154 \\
\hline Orange & 580,883 & 172,819 & 149,304 & 2,556 & 20,959 & 408,064 \\
\hline Osceola & 846,093 & 184,464 & 181,508 & 2,556 & 400 & 661,629 \\
\hline Palm Beach & $1,301,971$ & 122,420 & - & 122,420 & - & $1,179,551$ \\
\hline Pasco & 476,794 & 159,460 & 159,125 & 157 & 178 & 317,334 \\
\hline Pinellas & 179,302 & 18,128 & 6,460 & 7,677 & 3,991 & 161,174 \\
\hline Polk & $1,199,955$ & 283,287 & 243,155 & 27,013 & 13,119 & 916,668 \\
\hline Putnam & 462,189 & 350,717 & 347,781 & - & 2,936 & 111,472 \\
\hline Santa Rosa & 650,125 & 479,823 & 478,054 & - & 1,769 & 170,302 \\
\hline Sarasota & 365,920 & 59,591 & 48,801 & 1,132 & 9,658 & 306,329 \\
\hline Seminole & 197,261 & 74,473 & 72,545 & - & 1,928 & 122,788 \\
\hline St.Johns & 389,786 & 250,842 & 245,326 & 2,672 & 2,844 & 138,944 \\
\hline St. Lucie & 366,394 & 24,524 & 20,589 & 3,935 & - & 341,870 \\
\hline Sumter & 349,235 & 155,538 & 149,891 & 5,567 & 80 & 193,697 \\
\hline Suwannee & 440,115 & 233,667 & 231,514 & - & 2,153 & 206,448 \\
\hline Taylor & 666,848 & 582,767 & 579,435 & 3,332 & - & 84,081 \\
\hline Union & 153,792 & 121,390 & 121,390 & - & - & 32,402 \\
\hline Volusia & 707,763 & 461,469 & 440,936 & 8,425 & 12,108 & 246,294 \\
\hline Wakulla & 388,282 & 340,258 & 314,267 & - & 25,991 & 48,024 \\
\hline Walton & 676,909 & 522,237 & 516,781 & 2,633 & 2,823 & 154,672 \\
\hline Washington & 371,123 & 292,099 & 291,944 & - & 155 & 79,024 \\
\hline Total & $34,558,131$ & $16,221,197$ & $14,650,660$ & $1,047,861$ & 522,676 & $18,336,934$ \\
\hline
\end{tabular}


Table 2-Area of timberland, by county and ownership class, Florida, 1995

\begin{tabular}{|c|c|c|c|c|c|c|c|c|c|}
\hline \multirow[b]{3}{*}{ County } & \multirow{3}{*}{$\begin{array}{c}\text { All } \\
\text { ownerships }\end{array}$} & \multicolumn{8}{|c|}{ Ownership class } \\
\hline & & \multirow{2}{*}{$\begin{array}{c}\text { National } \\
\text { forest }\end{array}$} & \multirow{2}{*}{$\begin{array}{c}\text { Miscellaneous } \\
\text { Federal } \\
\end{array}$} & \multirow[b]{2}{*}{ State } & \multirow{2}{*}{$\begin{array}{c}\text { County and } \\
\text { municipal }\end{array}$} & \multirow{2}{*}{$\begin{array}{c}\text { Forest } \\
\text { industry }\end{array}$} & & Other private & \\
\hline & & & & & & & Farmer & Corporate & Individual \\
\hline & & & & & cres & & & & \\
\hline Alachua & 277,455 & - & 17 & 4,302 & 668 & 112,646 & 33,511 & 20,622 & 105,689 \\
\hline Baker & 326,523 & 86,832 & 3,678 & 186 & 40 & 194,779 & 5,468 & 10,935 & 24,605 \\
\hline Bay & 396,365 & - & 21,465 & 6,354 & 920 & 209,895 & - & 106,016 & 51,715 \\
\hline Bradford & 135,197 & - & - & 15,475 & 950 & 79,841 & 7,300 & 2,433 & 29,198 \\
\hline Brevard & 93,094 & - & 12,462 & 5,105 & 490 & - & 4,841 & 43,570 & 26,626 \\
\hline Broward & - & - & - & - & - & - & - & - & - \\
\hline Calhoun & 302,590 & - & 12 & 37 & 85 & 138,762 & 22,578 & 76,202 & 64,914 \\
\hline Charlotte & 30,289 & - & - & 7,114 & 200 & - & 1,532 & 16,848 & 4,595 \\
\hline Citrus & 185,932 & - & - & 48,530 & 706 & - & - & 49,211 & 87,485 \\
\hline Clay & 288,246 & - & 640 & 69,944 & 894 & 69,306 & 4,915 & 86,020 & 56,527 \\
\hline Collier & 190,458 & - & - & 8,851 & 200 & - & 22,327 & 19,536 & 139,544 \\
\hline Columbia & 357,483 & 80,091 & - & 5,093 & 412 & 142,382 & 34,689 & 34,689 & 60,127 \\
\hline Dade & - & - & - & - & - & - & - & - & - \\
\hline De Soto & 47,759 & - & - & 3,066 & 64 & - & 17,356 & 12,397 & 14,876 \\
\hline Dixie & 385,712 & - & 17,130 & 4,820 & 210 & 280,636 & 3,769 & 22,613 & 56,534 \\
\hline Duval & 229,614 & - & 16,774 & 4,345 & 7,730 & 41,420 & - & 86,424 & 72,921 \\
\hline Escambia & 250,789 & - & 2,950 & 5,314 & 90 & 102,287 & 11,212 & 61,665 & 67,271 \\
\hline Flagler & 237,592 & - & - & 1,000 & 650 & 163,806 & 22,387 & 29,849 & 19,900 \\
\hline Franklin & 301,493 & 19,678 & 7,319 & 45,421 & 646 & 104,859 & - & 107,094 & 16,476 \\
\hline Gadsden & 253,400 & - & 1 & 10,248 & 80 & 104,236 & 24,990 & 36,097 & 77,748 \\
\hline Gilchrist & 134,671 & - & - & - & 280 & 26,395 & 25,825 & 35,216 & 46,955 \\
\hline Glades & 91,648 & - & - & - & 60 & - & 2,862 & 88,726 & - \\
\hline Gulf & 310,232 & - & 755 & 35,123 & 55 & 252,459 & 1,680 & 6,720 & 13,440 \\
\hline Hamilton & 240,094 & - & - & 12,971 & 121 & 111,263 & 21,258 & 33,068 & 61.413 \\
\hline Hardee & 79,530 & - & - & 727 & 80 & - & 25,048 & 28,627 & 25,048 \\
\hline Hendry & 62,831 & - & - & 459 & - & - & - & 41,581 & 20,791 \\
\hline Hernando & 155,853 & - & 707 & 55,344 & 1,630 & - & - & 34,493 & 63,679 \\
\hline Highlands & 78,759 & - & 22,500 & 460 & 90 & - & 14,660 & 32,253 & 8,796 \\
\hline Hillsborough & 96,466 & - & - & 14,369 & 3,480 & - & 5,241 & 44,550 & 28,826 \\
\hline Holmes & 210,742 & - & 460 & 3,482 & 355 & 32,446 & 82,984 & 34,800 & 56,215 \\
\hline Indian River & 22,839 & - & - & 691 & 155 & - & - & 17,106 & 4,887 \\
\hline Jackson & 325,426 & - & 5,436 & 9,217 & 165 & 25,100 & 92,285 & 66,330 & 126,893 \\
\hline Jefferson & 284,399 & - & - & 14,031 & 30 & 163,420 & 25,157 & 25,157 & 56,604 \\
\hline Lafayette & 297,321 & - & - & 2,431 & 336 & 206,637 & 9,095 & 12,126 & 66,696 \\
\hline Lake & 248,490 & 69,407 & 280 & 20,081 & 750 & - & 14,582 & 48,607 & 94,783 \\
\hline Lee & 60,872 & - & - & 4,800 & 4,100 & - & - & 10,941 & 41,031 \\
\hline Leon & 305,389 & 103,735 & 20 & 8,126 & 1,928 & 54,450 & 11,428 & 62,851 & 62,851 \\
\hline Levy & 462,794 & - & 15,944 & 46,710 & 572 & 171,568 & 24,141 & 107,294 & 96,565 \\
\hline Liberty & 503,233 & 258,174 & - & 22,230 & 40 & 153,623 & 11,528 & 17,292 & 40,346 \\
\hline Madison & 326,368 & - & 728 & 6,867 & 10 & 121,465 & 53,809 & 10,249 & 133,240 \\
\hline Manatee & 40,003 & - & - & 2,870 & 6,321 & - & - & 18,487 & 12,325 \\
\hline Marion & 568,275 & 245,609 & - & 35,087 & 2,230 & 68,096 & 15,246 & 49,549 & 152,458 \\
\hline Martin & 40,647 & - & - & 11,854 & 300 & - & - & 14,247 & 14,246 \\
\hline Monroe & - & - & - & - & - & - & - & - & - \\
\hline Nassau & 323,374 & - & 5 & 5,840 & 599 & 209,466 & 15,352 & 17,911 & 74,201 \\
\hline Okaloosa & 448,276 & - & 211,310 & 59,766 & 748 & 55,134 & 22,747 & 15,165 & 83,406 \\
\hline Okeechobee & 43,331 & - & - & 692 & 100 & - & - & 28,359 & 14,180 \\
\hline Orange & 149,304 & - & 268 & 22,422 & 7,870 & - & 5,937 & 65,309 & 47,498 \\
\hline Osceola & 181,508 & - & 380 & 31,458 & 830 & - & 18,943 & 67,655 & 62,242 \\
\hline Palm Beach & - & - & - & - & - & - & - & - & - \\
\hline Pasco & 159,125 & - & 20 & 59,319 & 5,885 & - & 7,413 & 32,124 & 54,364 \\
\hline Pinellas & 6,460 & - & - & 1,350 & 1,231 & - & - & 2,586 & 1,293 \\
\hline Polk & 243,155 & - & 15,198 & 17,832 & 1,968 & - & 39,381 & 84,388 & 84,388 \\
\hline Putnam & 347,781 & 19,914 & 5,100 & 23,533 & 317 & 101,440 & 7,694 & 64,116 & 125,667 \\
\hline Santa Rosa & 478,054 & - & 56,300 & 130,375 & 809 & 165,986 & 21,156 & 11,753 & 91,675 \\
\hline Sarasota & 48,801 & - & - & 55 & 2,971 & - & 2,861 & 34,331 & 8,583 \\
\hline Seminole & 72,545 & - & 150 & 6,185 & 587 & - & 7,720 & 30,881 & 27,022 \\
\hline St. Johns & 245,326 & - & - & 4,616 & 138 & 136,287 & 6,801 & 38,540 & 58,944 \\
\hline St. Lucie & 20,589 & - & - & 225 & 200 & - & 2,521 & 10,082 & 7,561 \\
\hline Sumter & 149,891 & - & - & 78,216 & 10 & - & 8,431 & 16,862 & 46,372 \\
\hline Suwannee & 231,514 & - & - & 4,980 & 691 & 12,697 & 28,946 & 28,946 & 155,254 \\
\hline Taylor & 579,435 & - & - & 54,618 & 180 & 487,368 & 2,130 & 3,194 & 31,945 \\
\hline Union & 121,390 & - & - & 3,810 & 126 & 75,540 & 9,314 & 6,986 & 25,614 \\
\hline Volusia & 440,936 & - & 2,844 & 26,326 & 12,262 & 41,798 & 23,265 & 215,205 & 119,236 \\
\hline Wakulla & 314,267 & 146,082 & 28,385 & 147 & 1,213 & 54,094 & 5,817 & 11,634 & 66,895 \\
\hline Waiton & 516,781 & - & 137,233 & 36,102 & 209 & 103,406 & 57,559 & 62,356 & 119,916 \\
\hline Washington & 291,944 & - & - & 17,269 & 441 & 26,490 & 47,071 & 118,917 & 81,756 \\
\hline Total & $14,650,660$ & $1,029,522$ & 586,471 & $1,138,271$ & 77,508 & $4,601,483$ & 994,763 & $2,629,791$ & $3,592,851$ \\
\hline
\end{tabular}


Table 3-Area of timberland, by county and forest-type group, Florida, 1995

\begin{tabular}{|c|c|c|c|c|c|c|c|}
\hline \multirow[b]{2}{*}{ County } & \multirow[b]{2}{*}{$\begin{array}{c}\text { All type } \\
\text { groups }\end{array}$} & \multicolumn{6}{|c|}{ Forest-type group } \\
\hline & & $\begin{array}{l}\text { Longleaf- } \\
\text { slash }\end{array}$ & $\begin{array}{l}\text { Loblolly- } \\
\text { shortleaf }\end{array}$ & $\begin{array}{l}\text { Oak- } \\
\text { pine }\end{array}$ & $\begin{array}{c}\text { Oak- } \\
\text { hickory }\end{array}$ & $\begin{array}{c}\text { Oak-gum- } \\
\text { cypress }\end{array}$ & $\begin{array}{l}\text { Elm-ash- } \\
\text { cottonwood }\end{array}$ \\
\hline & & & & Acres & & & \\
\hline Alachua & 277,455 & 131,836 & 16,709 & 18,904 & 72,826 & 37,180 & - \\
\hline Baker & 326,523 & 219,110 & 6,411 & 12,321 & 2,734 & 85,947 & - \\
\hline Bay & 396,365 & 283,516 & 40,263 & 19,583 & 17,634 & 35,369 & - \\
\hline Bradford & 135,197 & 85,742 & 7,909 & 9,422 & 9,712 & 22,412 & - \\
\hline Brevard & 93,094 & 29,435 & 7,263 & 2,421 & 21,999 & 31,976 & - \\
\hline Broward & - & - & - & - & - & - & - \\
\hline Calhoun & 302,590 & 137,989 & 45,729 & 31,717 & 27,791 & 56,971 & 2,393 \\
\hline Charlotte & 30,289 & 13,985 & - & - & - & 16,304 & - \\
\hline Citrus & 185,932 & 34,005 & 8,202 & 42,936 & 62,050 & 38,739 & - \\
\hline Clay & 288,246 & 149,549 & 17,022 & 21,102 & 49,370 & 51,203 & - \\
\hline Collier & 190,458 & 28,107 & - & 8,372 & 10,008 & 143,971 & - \\
\hline Columbia & 357,483 & 174,896 & 20,950 & 19,274 & 54,198 & 88,165 & - \\
\hline Dade & - & - & - & - & - & - & - \\
\hline De Soto & 47,759 & 9,916 & - & - & 23,913 & 13,930 & - \\
\hline Dixie & 385,712 & 158,165 & 9,846 & 13,617 & 41,546 & 162,538 & - \\
\hline Duval & 229,614 & 99,356 & 26,239 & 34,002 & 31,643 & 38,374 & - \\
\hline Escambia & 250,789 & 117,544 & 42,239 & 33,090 & 19,511 & 38,405 & - \\
\hline Flagler & 237,592 & 138,542 & 9,254 & 19,150 & 16,122 & 54,524 & - \\
\hline Franklin & 301,493 & 188,745 & 22,356 & 24,330 & 5,492 & 60,570 & - \\
\hline Gadsden & 253,400 & 39,471 & 77,263 & 34,793 & 49,549 & 52,324 & - \\
\hline Gilchrist & 134,671 & 73,021 & - & 16,434 & 31,477 & 13,739 & - \\
\hline Glades & 91,648 & 45,853 & - & 2,862 & 5,726 & 37,207 & - \\
\hline Gulf & 310,232 & 177,937 & 4,836 & 15,983 & 6,515 & 103,281 & 1,680 \\
\hline Hamilton & 240,094 & 106,258 & 11,768 & 31,467 & 31,708 & 58,893 & - \\
\hline Hardee & 79,530 & 17,892 & - & 3,579 & 28,704 & 29,355 & - \\
\hline Hendry & 62,831 & 12,934 & - & 4,158 & 4,158 & 41,581 & - \\
\hline Hernando & 155,853 & 31,013 & 17,550 & 20,540 & 65,646 & 21,104 & - \\
\hline Highlands & 78,759 & 18,796 & - & 14,228 & 16,818 & 28,917 & - \\
\hline Hillsborough & 96,466 & 18,344 & - & 10,483 & 11,876 & 55,763 & - \\
\hline Holmes & 210,742 & 53,418 & 47,258 & 31,648 & 32,582 & 45,836 & - \\
\hline Indian River & 22,839 & 12,218 & - & 2,444 & 2,444 & 5,733 & - \\
\hline Jackson & 325,426 & 64,093 & 75,033 & 29,981 & 77,864 & 75,571 & 2,884 \\
\hline Jefferson & 284,399 & 78,921 & 54,871 & 22,668 & 33,146 & 94,793 & - \\
\hline Lafayette & 297,321 & 141,709 & 9,266 & 34,462 & 32,562 & 79,322 & - \\
\hline Lake & 248,490 & 58,663 & 40,558 & 27,088 & 32,187 & 89,994 & - \\
\hline Lee & 60,872 & 29,755 & - & 5,470 & 2,736 & 22,911 & - \\
\hline Leon & 305,389 & 93,532 & 64,562 & 56,860 & 48,206 & 42,229 & - \\
\hline Levy & 462,794 & 152,206 & 60,441 & 41,250 & 88,709 & 117,421 & 2,767 \\
\hline Liberty & 503,233 & 243,614 & 52,869 & 21,559 & 13,682 & 163,226 & 8,283 \\
\hline Madison & 326,368 & 112,023 & 34,158 & 20,776 & 59,357 & 97,492 & 2,562 \\
\hline Manatee & 40,003 & 13,299 & 2,054 & 2,054 & 10,270 & 12,326 & - \\
\hline Marion & 568,275 & 95,461 & 200,928 & 76,925 & 134,427 & 60,534 & - \\
\hline Martin & 40,647 & 26,400 & 2,850 & 2,850 & 2,849 & 5,698 & - \\
\hline Monroe & - & - & - & - & - & - & - \\
\hline Nassau & 323,374 & 152,879 & 22,252 & 30,289 & 29,937 & 88,017 & - \\
\hline Okaloosa & 448,276 & 183,061 & 55,055 & 94,648 & 62,165 & 53,347 & - \\
\hline Okeechobee & 43,331 & 11,344 & - & 2,836 & - & 29,151 & - \\
\hline Orange & 149,304 & 26,241 & 20,780 & 11,874 & 17,810 & 72,599 & - \\
\hline Osceola & 181,508 & 27,932 & 2,706 & 18,024 & 19,772 & 113,074 & - \\
\hline Palm Beach & - & - & - & - & - & - & - \\
\hline Pasco & 159,125 & 37,850 & 2,471 & 16,129 & 39,019 & 63,656 & - \\
\hline Pinellas & 6,460 & - & 1,293 & 1,293 & - & 3,874 & - \\
\hline Polk & 243,155 & 62,681 & 5,361 & 18,777 & 54,494 & 101,842 & - \\
\hline Putnam & 347,781 & 157,789 & 42,705 & 37,044 & 57,379 & 49,677 & 3,187 \\
\hline Santa Rosa & 478,054 & 202,709 & 38,453 & 105,475 & 47,797 & 83,620 & - \\
\hline Sarasota & 48,801 & 23,737 & - & 424 & 9,007 & 15,633 & - \\
\hline Seminole & 72,545 & 11,731 & - & 15,441 & 11,580 & 33,793 & - \\
\hline St. Johns & 245,326 & 120,436 & 17,057 & 32,576 & 6,801 & 68,456 & - \\
\hline St. Lucie & 20,589 & 12,603 & 200 & 2,520 & - & 5,266 & - \\
\hline Sumter & 149,891 & 43,938 & 12,656 & 8,126 & 41,545 & 39,410 & 4,216 \\
\hline Suwannee & 231,514 & 113,556 & 10,527 & 17,871 & 71,099 & 18,461 & - \\
\hline Taylor & 579,435 & 318,311 & 30,784 & 23,997 & 37,995 & 159,673 & 8,675 \\
\hline Union & 121,390 & 84,214 & 4,657 & 4,902 & 2,328 & 25,289 & - \\
\hline Volusia & 440,936 & 182,312 & 23,264 & 66,625 & 34,150 & 131,676 & 2,909 \\
\hline Wakulla & 314,267 & 154,668 & 36,681 & 35,303 & 38,915 & 48,700 & - \\
\hline Walton & 516,781 & 172,472 & 127,556 & 57,290 & 79,863 & 79,600 & - \\
\hline Washington & 291,944 & 53,611 & 75,329 & 36,713 & 61,940 & 61,884 & 2,467 \\
\hline Total & $14,650,660$ & $5,871,344$ & $1,566,444$ & $1,478,980$ & $2,013,343$ & $3,678,526$ & 42,023 \\
\hline
\end{tabular}


Table 4-Area of timberland, by county and stand-size class, Florida, 1995

\begin{tabular}{|c|c|c|c|c|c|}
\hline \multirow[b]{2}{*}{ County } & \multirow[b]{2}{*}{$\begin{array}{c}\text { All } \\
\text { stands }\end{array}$} & \multicolumn{3}{|c|}{ Stand-size class } & \multirow[b]{2}{*}{$\begin{array}{c}\text { Nonstocked } \\
\text { areas }\end{array}$} \\
\hline & & Sawtimber & Poletimber & $\begin{array}{l}\text { Sapling- } \\
\text { seedling }\end{array}$ & \\
\hline & \multicolumn{5}{|c|}{ Acres } \\
\hline Alachua & 277,455 & 88,921 & 89,716 & 93,582 & 5,236 \\
\hline Baker & 326,523 & 79,872 & 104,739 & 123,394 & 18,518 \\
\hline Bay & 396,365 & 34,808 & 103,896 & 238,079 & 19,582 \\
\hline Bradford & 135,197 & 29,112 & 52,190 & 51,462 & 2,433 \\
\hline Brevard & 93,094 & 46,308 & 27,349 & 14,595 & 4,842 \\
\hline Broward & - & - & - & - & - \\
\hline Calhoun & 302,590 & 78,508 & 73,433 & 142,182 & 8,467 \\
\hline Charlotte & 30,289 & 13,786 & 10,377 & 1,532 & 4,594 \\
\hline Citrus & 185,932 & 54,877 & 48,672 & 82,383 & - \\
\hline Clay & 288,246 & 86,425 & 101,929 & 97,434 & 2,458 \\
\hline Collier & 190,458 & 85,361 & 63,235 & 27,907 & 13,955 \\
\hline Columbia & 357,483 & 94,055 & 125,078 & 135,903 & 2,447 \\
\hline Dade & - & - & - & - & - \\
\hline De Soto & 47,759 & 31,287 & 4,077 & 4,958 & 7,437 \\
\hline Dixie & 385,712 & 138,595 & 156,640 & 78,929 & 11,548 \\
\hline Duval & 229,614 & 88,688 & 64,762 & 71,530 & 4,634 \\
\hline Escambia & 250,789 & 104,111 & 64,227 & 82,451 & - \\
\hline Flagler & 237,592 & 83,417 & 78,057 & 70,600 & 5,518 \\
\hline Franklin & 301,493 & 57,775 & 116,503 & 106,306 & 20,909 \\
\hline Gadsden & 253,400 & 60,372 & 88,434 & 104,594 & - \\
\hline Gilchrist & 134,671 & 24,000 & 58,494 & 52,177 & - \\
\hline Glades & 91,648 & 34,347 & 17,232 & 20,034 & 20,035 \\
\hline Gulf & 310,232 & 82,721 & 69,836 & 145,053 & 12,622 \\
\hline Hamilton & 240,094 & 36,158 & 103,239 & 95,488 & 5,209 \\
\hline Hardee & 79,530 & 53,675 & 17,891 & 7,964 & - \\
\hline Hendry & 62,831 & 33,264 & 17,092 & 8,317 & 4,158 \\
\hline Hernando & 155,853 & 68,452 & 45,909 & 38,839 & 2,653 \\
\hline Highlands & 78,759 & 33,024 & 7,932 & 26,507 & 11,296 \\
\hline Hillsborough & 96,466 & 69,318 & 18,591 & 5,936 & 2,621 \\
\hline Holmes & 210,742 & 48,393 & 39,702 & 122,647 & - \\
\hline Indian River & 22,839 & 15,354 & 5,042 & - & 2,443 \\
\hline Jackson & 325,426 & 80,260 & 58,657 & 186,509 & - \\
\hline Jefferson & 284,399 & 120,534 & 55,095 & 104,969 & 3,801 \\
\hline Lafayette & 297,321 & 64,566 & 57,558 & 155,893 & 19,304 \\
\hline Lake & 248,490 & 92,871 & 50,425 & 98,507 & 6,687 \\
\hline Lee & 60,872 & 9,570 & 29,419 & 19,148 & 2,735 \\
\hline Leon & 305,389 & 122,740 & 59,500 & 117,699 & 5,450 \\
\hline Levy & 462,794 & 170,964 & 150,727 & 130,430 & 10,673 \\
\hline Liberty & 503,233 & 220,330 & 99,930 & 169,468 & 13,505 \\
\hline Madison & 326,368 & 69,881 & 106,069 & 128,488 & 21,930 \\
\hline Manatee & 40,003 & 18,488 & 4,108 & 13,299 & 4,108 \\
\hline Marion & 568,275 & 192,209 & 136,052 & 225,124 & 14,890 \\
\hline Martin & 40,647 & 20,172 & 17,625 & 2,850 & - \\
\hline Monroe & - & - & - & - & - \\
\hline Nassau & 323,374 & 89,372 & 106,526 & 113,251 & 14,225 \\
\hline Okaloosa & 448,276 & 149,682 & 128,383 & 167,467 & 2,744 \\
\hline Okeechobee & 43,331 & 28,359 & 11,444 & 3,528 & - \\
\hline Orange & 149,304 & 83,495 & 22,315 & 40,525 & 2,969 \\
\hline Osceola & 181,508 & 91,470 & 34,265 & 53,067 & 2,706 \\
\hline Palm Beach & - & - & - & - & - \\
\hline Pasco & 159,125 & 82,256 & 33,426 & 37,199 & 6,244 \\
\hline Pinellas & 6,460 & 6,460 & - & - & - \\
\hline Polk & 243,155 & 97,467 & 55,804 & 84,524 & 5,360 \\
\hline Putnam & 347,781 & 90,291 & 103,981 & 140,063 & 13,446 \\
\hline Santa Rosa & 478,054 & 170,947 & 113,472 & 193,635 & - \\
\hline Sarasota & 48,801 & 29,513 & 10,280 & 425 & 8,583 \\
\hline Seminole & 72,545 & 53,245 & 7,720 & 11,580 & - \\
\hline St. Johns & 245,326 & 67,770 & 84,641 & 90,259 & 2,656 \\
\hline St. Lucie & 20,589 & 15,348 & - & 5,241 & - \\
\hline Sumter & 149,891 & 80,959 & 34,168 & 32,808 & 1,956 \\
\hline Suwannee & 231,514 & 62,604 & 89,370 & 79,540 & - \\
\hline Taylor & 579,435 & 109,636 & 163,690 & 284,482 & 21,627 \\
\hline Union & 121,390 & 29,910 & 42,946 & 44,724 & 3,810 \\
\hline Volusia & 440,936 & 162,760 & 122,963 & 125,974 & 29,239 \\
\hline Wakulla & 314,267 & 124,927 & 62,071 & 117,135 & 10,134 \\
\hline Walton & 516,781 & 149,269 & 113,492 & 251,243 & 2,777 \\
\hline Washington & 291,944 & 73,371 & 83,650 & 132,445 & 2,478 \\
\hline Total & $14,650,660$ & $4,786,680$ & $4,024,046$ & $5,412,282$ & 427,652 \\
\hline
\end{tabular}


Table 5-Area of timberland, by county and site class, Florida, 1995

\begin{tabular}{|c|c|c|c|c|c|c|}
\hline \multirow[b]{2}{*}{ County } & \multirow{2}{*}{$\begin{array}{c}\text { All } \\
\text { classes }\end{array}$} & \multicolumn{5}{|c|}{ Site class (cubic feet per acre per year) } \\
\hline & & $>164$ & $120-164$ & $85-119$ & $50-84$ & $20-49$ \\
\hline & \multicolumn{6}{|c|}{ Acres } \\
\hline Alachua & 277,455 & - & 10,439 & 101,707 & 144,968 & 20,341 \\
\hline Baker & 326,523 & - & 10,452 & 60,381 & 227,800 & 27,890 \\
\hline Bay & 396,365 & - & - & 2,956 & 255,197 & 138,212 \\
\hline Bradford & 135,197 & - & 901 & 19,427 & 102,189 & 12,680 \\
\hline Brevard & 93,094 & - & - & 7,261 & 46,132 & 39,701 \\
\hline Broward & - & - & - & - & - & - \\
\hline Calhoun & 302,590 & - & 7,178 & 43,682 & 204,357 & 47,373 \\
\hline Charlotte & 30,289 & - & - & 1,532 & 13,784 & 14,973 \\
\hline Citrus & 185,932 & - & - & 9,936 & 63,540 & 112,456 \\
\hline Clay & 288,246 & - & 4,870 & 44,920 & 178,306 & 60,150 \\
\hline Collier & 190,458 & - & - & 2,791 & 69,769 & 117,898 \\
\hline Columbia & 357,483 & - & 7,432 & 92,062 & 225,690 & 32,299 \\
\hline Dade & - & - & - & - & - & - \\
\hline De Soto & 47,759 & - & - & - & 23,847 & 23,912 \\
\hline Dixie & 385,712 & - & 4,923 & 53,610 & 264,599 & 62,580 \\
\hline Duval & 229,614 & 1,932 & 6,056 & 36,764 & 130,758 & 54,104 \\
\hline Escambia & 250,789 & 2,803 & - & 27,484 & 199,316 & 21,186 \\
\hline Flagler & 237,592 & - & - & 29,398 & 170,733 & 37,461 \\
\hline Franklin & 301,493 & - & - & 8,876 & 108,965 & 183,652 \\
\hline Gadsden & 253,400 & - & 2,049 & 63,000 & 161,015 & 27,336 \\
\hline Gilchrist & 134,671 & - & 3,306 & 16,148 & 82,350 & 32,867 \\
\hline Glades & 91,648 & - & - & - & 54,379 & 37,269 \\
\hline Gulf & 310,232 & - & - & 9,672 & 153,483 & 147,077 \\
\hline Hamilton & 240,094 & - & - & 36,553 & 186,587 & 16,954 \\
\hline Hardee & 79,530 & - & - & 10,735 & 36,511 & 32,284 \\
\hline Hendry & 62,831 & - & - & - & 33,265 & 29,566 \\
\hline Hernando & 155,853 & - & 5,169 & 13,266 & 100,685 & 36,733 \\
\hline Highlands & 78,759 & - & - & - & 45,212 & 33,547 \\
\hline Hillsborough & 96,466 & - & - & 8,557 & 73,414 & 14,495 \\
\hline Holmes & 210,742 & - & 5,354 & 63,294 & 115,461 & 26,633 \\
\hline Indian River & 22,839 & - & - & - & 8,023 & 14,816 \\
\hline Jackson & 325,426 & - & 10,583 & 57,679 & 224,674 & 32,490 \\
\hline Jefferson & 284,399 & - & 8,385 & 60,465 & 187,449 & 28,100 \\
\hline Lafayette & 297,321 & - & 3,032 & 24,744 & 224,384 & 45,161 \\
\hline Lake & 248,490 & 2,431 & 9,357 & 35,964 & 136,825 & 63,913 \\
\hline Lee & 60,872 & - & - & - & 10,942 & 49,930 \\
\hline Leon & 305,389 & - & 2,857 & 56,959 & 162,364 & 83,209 \\
\hline Levy & 462,794 & - & 10,727 & 86,037 & 256,068 & 109,962 \\
\hline Liberty & 503,233 & - & - & 43,845 & 277,282 & 182,106 \\
\hline Madison & 326,368 & - & - & 68,026 & 225,437 & 32,905 \\
\hline Manatee & 40,003 & - & - & - & 23,570 & 16,433 \\
\hline Marion & 568,275 & 2,064 & 17,509 & 112,920 & 286,686 & 149,096 \\
\hline Martin & 40,647 & - & - & - & 14,475 & 26,172 \\
\hline Monroe & - & - & - & - & - & - \\
\hline Nassau & 323,374 & - & 8,389 & 52,012 & 198,769 & 64,204 \\
\hline Okaloosa & 448,276 & - & 5,488 & 34,641 & 196,267 & 211,880 \\
\hline Okeechobee & 43,331 & - & - & 2,836 & 34,723 & 5,772 \\
\hline Orange & 149,304 & - & - & 2,969 & 94,144 & 52,191 \\
\hline Osceola & 181,508 & - & - & 8,118 & 120,275 & 53,115 \\
\hline Palm Beach & - & - & - & - & - & - \\
\hline Pasco & 159,125 & - & - & 9,366 & 103,327 & 46,432 \\
\hline Pinellas & 6,460 & - & - & - & 5,167 & 1,293 \\
\hline Polk & 243,155 & - & - & 656 & 168,251 & 74,248 \\
\hline Putnam & 347,781 & - & - & 78,797 & 186,809 & 82,175 \\
\hline Santa Rosa & 478,054 & - & 22,935 & 98,462 & 246,191 & 110,466 \\
\hline Sarasota & 48,801 & - & - & - & 28,350 & 20,451 \\
\hline Seminole & 72,545 & - & - & 19,301 & 45,524 & 7,720 \\
\hline St. Johns & 245,326 & 2,267 & - & 30,695 & 186,976 & 25,388 \\
\hline St. Lucie & 20,589 & - & - & - & 5,265 & 15,324 \\
\hline Sumter & 149,891 & 4,216 & 1,955 & 12,039 & 105,043 & 26,638 \\
\hline Suwannee & 231,514 & - & 2,631 & 50,044 & 144,910 & 33,929 \\
\hline Taylor & 579,435 & - & 2,971 & 63,016 & 368,137 & 145,311 \\
\hline Union & 121,390 & - & - & 24,889 & 84,366 & 12,135 \\
\hline Volusia & 440,936 & 2,908 & 5,816 & 45,708 & 298,374 & 88,130 \\
\hline Wakulla & 314,267 & - & - & 36,347 & 169,607 & 108,313 \\
\hline Walton & 516,781 & - & - & 28,335 & 291,469 & 196,977 \\
\hline Washington & 291,944 & - & 6,542 & 35,385 & 109,701 & 140,316 \\
\hline Total & $14,650,660$ & 18,621 & 187,306 & $1,944,267$ & $8,702,136$ & $3,798,330$ \\
\hline
\end{tabular}


Table 6-Area of timberland, by county and stocking class of growing-stock trees, Florida, 1995

\begin{tabular}{|c|c|c|c|c|c|c|}
\hline \multirow[b]{2}{*}{ County } & \multirow{2}{*}{$\begin{array}{c}\text { All } \\
\text { classes }\end{array}$} & \multicolumn{5}{|c|}{ Stocking class (percent) ${ }^{a}$} \\
\hline & & $>130$ & $100-130$ & $60-99$ & $16.7-59$ & $<16.7$ \\
\hline & \multicolumn{6}{|c|}{ Acres } \\
\hline Alachua & 277,455 & 23,457 & 81,757 & 94,588 & 59,527 & 18,126 \\
\hline Baker & 326,523 & 31,270 & 127,893 & 108,801 & 33,687 & 24,872 \\
\hline Bay & 396,365 & 5,913 & 123,420 & 160,826 & 75,911 & 30,295 \\
\hline Bradford & 135,197 & 19,088 & 41,339 & 42,646 & 27,889 & 4,235 \\
\hline Brevard & 93,094 & 7,260 & 7,261 & 26,627 & 22,175 & 29,771 \\
\hline Broward & - & - & - & - & - & - \\
\hline Calhoun & 302,590 & 5,215 & 62,611 & 150,386 & 54,623 & 29,755 \\
\hline Charlotte & 30,289 & 3,063 & 4,596 & 8,646 & 3,264 & 10,720 \\
\hline Citrus & 185,932 & 4,468 & 9,935 & 50,406 & 91,317 & 29,806 \\
\hline Clay & 288,246 & 8,952 & 55,582 & 128,547 & 60,987 & 34,178 \\
\hline Collier & 190,458 & 16,745 & 25,118 & 33,490 & 68,818 & 46,287 \\
\hline Columbia & 357,483 & 19,845 & 122,011 & 144,088 & 64,110 & 7,429 \\
\hline Dade & - & - & - & - & - & - \\
\hline De Soto & 47,759 & - & 6,491 & 4,959 & 16,473 & 19,836 \\
\hline Dixie & 385,712 & 4,924 & 90,265 & 155,651 & 104,786 & 30,086 \\
\hline Duval & 229,614 & 10,928 & 83,663 & 87,741 & 31,844 & 15,438 \\
\hline Escambia & 250,789 & 11,103 & 103,848 & 91,645 & 41,390 & 2,803 \\
\hline Flagler & 237,592 & 14,579 & 68,460 & 98,839 & 45,166 & 10,548 \\
\hline Franklin & 301,493 & 3,659 & 82,369 & 135,181 & 49,457 & 30,827 \\
\hline Gadsden & 253,400 & 9,501 & 76,457 & 127,459 & 39,983 & - \\
\hline Gilchrist & 134,671 & 2,348 & 38,803 & 43,260 & 43,216 & 7,044 \\
\hline Glades & 91,648 & 2,862 & 14,310 & 17,172 & 20,094 & 37,210 \\
\hline Gulf & 310,232 & 21,175 & 41,027 & 130,826 & 88,056 & 29,148 \\
\hline Hamilton & 240,094 & 20,244 & 80,901 & 92,307 & 33,861 & 12,781 \\
\hline Hardee & 79,530 & - & 15,041 & 21,471 & 28,626 & 14,392 \\
\hline Hendry & 62,831 & 12,474 & 12,475 & 8,317 & 17,091 & 12,474 \\
\hline Hernando & 155,853 & 2,515 & 23,149 & 52,240 & 67,611 & 10,338 \\
\hline Highlands & 78,759 & 2,500 & 11,756 & 19,660 & 22,161 & 22,682 \\
\hline Hillsborough & 96,466 & 20,511 & 10,482 & 27,842 & 29,769 & 7,862 \\
\hline Holmes & 210,742 & 12,908 & 55,305 & 94,360 & 45,032 & 3,137 \\
\hline Indian River & 22,839 & 691 & 2,444 & 2,444 & 9,775 & 7,485 \\
\hline Jackson & 325,426 & 8,652 & 105,802 & 145,986 & 47,684 & 17,302 \\
\hline Jefferson & 284,399 & 18,008 & 63,890 & 141,735 & 52,771 & 7,995 \\
\hline Lafayette & 297,321 & 5,424 & 72,142 & 104,235 & 74,520 & 41,000 \\
\hline Lake & 248,490 & 8,512 & 49,715 & 78,618 & 86,485 & 25,160 \\
\hline Lee & 60,872 & - & 10,941 & 10,608 & 33,853 & 5,470 \\
\hline Leon & 305,389 & 2,857 & 82,721 & 164,355 & 50,006 & 5,450 \\
\hline Levy & 462,794 & 34,941 & 90,531 & 172,671 & 110,893 & 53,758 \\
\hline Liberty & 503,233 & 26,367 & 78,138 & 222,629 & 154,218 & 21,881 \\
\hline Madison & 326,368 & 21,056 & 49,671 & 121,154 & 94,201 & 40,286 \\
\hline Manatee & 40,003 & - & - & 11,088 & 18,645 & 10,270 \\
\hline Marion & 568,275 & 8,317 & 108,248 & 196,976 & 174,445 & 80,289 \\
\hline Martin & 40,647 & - & 2,850 & 11,625 & 20,473 & 5,699 \\
\hline Monroe & - & - & - & - & - & - \\
\hline Nassau & 323,374 & 29,156 & 102,899 & 130,378 & 40,886 & 20,055 \\
\hline Okaloosa & 448,276 & 17,911 & 43,315 & 177,709 & 182,470 & 26,871 \\
\hline Okeechobee & 43,331 & 2,836 & 14,871 & 11,344 & 8,508 & 5,772 \\
\hline Orange & 149,304 & 17,337 & 22,793 & 37,638 & 45,774 & 25,762 \\
\hline Osceola & 181,508 & 24,354 & 44,548 & 48,665 & 49,581 & 14,360 \\
\hline Palm Beach & - & - & - & - & - & - \\
\hline Pasco & 159,125 & 15,477 & 21,742 & 51,800 & 43,443 & 26,663 \\
\hline Pinellas & 6,460 & - & 1,293 & 3,874 & 1,293 & - \\
\hline Polk & 243,155 & 22,237 & 40,102 & 59,935 & 82,031 & 38,850 \\
\hline Putnam & 347,781 & 14,967 & 63,915 & 109,692 & 100,517 & 58,690 \\
\hline Santa Rosa & 478,054 & 39,636 & 80,188 & 199,269 & 153,599 & 5,362 \\
\hline Sarasota & 48,801 & - & 2,916 & 8,582 & 16,427 & 20,876 \\
\hline Seminole & 72,545 & 6,185 & 4,010 & 19,300 & 30,882 & 12,168 \\
\hline St. Johns & 245,326 & 17,093 & 69,945 & 113,386 & 37,712 & 7,190 \\
\hline St. Lucie & 20,589 & 225 & - & 5,041 & 12,803 & 2,520 \\
\hline Sumter & 149,891 & 13,992 & 23,474 & 54,625 & 47,719 & 10,081 \\
\hline Suwannee & 231,514 & 2,631 & 73,132 & 100,489 & 42,103 & 13,159 \\
\hline Taylor & 579,435 & 9,738 & 142,765 & 220,784 & 144,839 & 61,309 \\
\hline Union & 121,390 & 10,297 & 42,421 & 50,398 & 14,464 & 3,810 \\
\hline Volusia & 440,936 & 24,551 & 103,914 & 141,384 & 110,576 & 60,511 \\
\hline Wakulla & 314,267 & 7,581 & 66,957 & 135,160 & 89,517 & 15,052 \\
\hline Walton & 516,781 & 35,821 & 95,934 & 213,027 & 131,577 & 40,422 \\
\hline Washington & 291,944 & 4,954 & 76,218 & 112,584 & 68,456 & 29,732 \\
\hline Total & $14,650,660$ & 749,311 & $3,330,770$ & $5,547,169$ & $3,670,070$ & $1,353,340$ \\
\hline
\end{tabular}

"See stocking standards under "stocking" in definitions. 
Table 7-Volume of growing stock and sawtimber on timberland, by county and species group, Florida, 1995

\begin{tabular}{|c|c|c|c|c|c|c|c|c|c|c|}
\hline \multirow[b]{2}{*}{ County } & \multicolumn{5}{|c|}{ Growing stock } & \multicolumn{5}{|c|}{ Sawtimber } \\
\hline & $\begin{array}{c}\text { All } \\
\text { species }\end{array}$ & Pine & $\begin{array}{c}\text { Other } \\
\text { softwood }\end{array}$ & $\begin{array}{c}\text { Soft } \\
\text { hardwood }\end{array}$ & $\begin{array}{c}\text { Hard } \\
\text { hardwood }\end{array}$ & $\begin{array}{c}\text { All } \\
\text { species }\end{array}$ & Pine & $\begin{array}{c}\text { Other } \\
\text { softwood }\end{array}$ & $\begin{array}{c}\text { Soft } \\
\text { hardwood }\end{array}$ & $\begin{array}{c}\text { Hard } \\
\text { hardwood }\end{array}$ \\
\hline & \multicolumn{5}{|c|}{ Thousand cubic feet } & \multicolumn{5}{|c|}{ Thousand board feet } \\
\hline Alachua & 308,656 & 149,938 & 40,370 & 46,796 & 71,552 & 817,086 & 267,018 & 155,342 & 121,203 & 273,523 \\
\hline Baker & 381,312 & 236,892 & 62,373 & 78,086 & 3,961 & $1,051,663$ & 681,372 & 189,709 & 171,136 & 9,446 \\
\hline Bay & 174,121 & 133,129 & 7,387 & 22,197 & 11,408 & 234,051 & 136,554 & 16,121 & 63,793 & 17,583 \\
\hline Bradford & 126,652 & 84,016 & 7,979 & 20,201 & 14,456 & 265,107 & 164,248 & 17,720 & 24,474 & 58,665 \\
\hline Brevard & 85,753 & 47,428 & 5,138 & 14,832 & 18,355 & 252,920 & 108,130 & 15,037 & 57,312 & 72,441 \\
\hline Broward & - & - & - & - & - & - & - & - & - & - \\
\hline Calhoun & 256,289 & 149,242 & 16,966 & 58,035 & 32,046 & 739,235 & 384,875 & 65,092 & 168,247 & 121,021 \\
\hline Charlotte & 31,223 & 13,864 & 16,626 & - & 733 & 74,487 & 37,823 & 33,736 & - & 2,928 \\
\hline Citrus & 140,779 & 46,365 & 33,193 & 27,683 & 33,538 & 474,604 & 157,317 & 137,452 & 79,542 & 100,293 \\
\hline Clay & 293,876 & 173,164 & 12,653 & 57,541 & 50,518 & 789,405 & 464,342 & 46,266 & 120,606 & 158,191 \\
\hline Collier & 191,337 & 36,363 & 138,516 & 11,367 & 5,091 & 587,383 & 121,926 & 426,028 & 23,130 & 16,299 \\
\hline Columbia & 393,030 & 206,880 & 50,195 & 94,692 & 41,263 & $1.069,516$ & 643,743 & 163,235 & 147,093 & 115,445 \\
\hline Dade & - & - & - & - & - & - & - & - & - & - \\
\hline De Soto & 38,975 & 3,559 & 5,964 & 19,609 & 9,843 & 142,442 & 18,593 & 23,561 & 65,308 & 34,980 \\
\hline Dixie & 434,851 & 173,362 & 79,456 & 88,372 & 93,661 & $1,040,543$ & 348,482 & 223,128 & 193,695 & 275,238 \\
\hline Duval & 323,561 & 175,058 & 14,873 & 84,688 & 48,942 & $1,009,843$ & 556,677 & 50,288 & 220,930 & 181,948 \\
\hline Escambia & 330,664 & 212,751 & 5,358 & 83,585 & 28,970 & $1,087,781$ & 698,577 & 16,118 & 277,315 & 95,771 \\
\hline Flagler & 307,061 & 154,067 & 77,335 & 51,680 & 23,979 & 906,656 & 430,800 & 227,515 & 141,760 & 106,581 \\
\hline Franklin & 274,987 & 166,088 & 44,700 & 54,530 & 9,669 & 667,220 & 340,083 & 133,051 & 168,420 & 25,666 \\
\hline Gadsden & 284,622 & 120,369 & - & 89,787 & 74,466 & 867,984 & 363,777 & - & 250,297 & 253,910 \\
\hline Gilchrist & 116,283 & 74,812 & 17,628 & 5,159 & 18,684 & 224,520 & 107,459 & 49,730 & 4,697 & 62,634 \\
\hline Glades & 52,991 & 13,911 & 32,621 & 4,658 & 1,801 & 191,785 & 46,219 & 126,549 & 10,051 & 8,966 \\
\hline Gulf & 246,731 & 73,493 & 57,821 & 97,372 & 18,045 & 700,273 & 173,614 & 176,370 & 288,927 & 61,362 \\
\hline Hamilton & 251,721 & 114,103 & 24,201 & 57,233 & 56,184 & 561,533 & 207,276 & 50,824 & 97,697 & 205,736 \\
\hline Hardee & 99,145 & 30,763 & 25,158 & 15,159 & 28,065 & 365,314 & 152,606 & 82,131 & 27,824 & 102,753 \\
\hline Hendry & 102,080 & 4,742 & 89,060 & 2,624 & 5,654 & 365,354 & 19,748 & 309,863 & 11,962 & 23,781 \\
\hline Hernando & 191,244 & 68,293 & 6,924 & 52,465 & 63,562 & 611,191 & 225,121 & 31,261 & 150,450 & 204,359 \\
\hline Highlands & 71,945 & 16,288 & 31,278 & 15,824 & 8,555 & 263,045 & 64,994 & 129,131 & 34,385 & 34,535 \\
\hline Hillsborough & 196,645 & 25,407 & 96,564 & 32,771 & 41,903 & 594,463 & 113,266 & 249,075 & 94,904 & 137,218 \\
\hline Holmes & 177,667 & 71,737 & 8,661 & 70,625 & 26,644 & 462,143 & 221,501 & 32,421 & 128,889 & 79,332 \\
\hline Indian River & 21,046 & 12,804 & 6,527 & 236 & 1,479 & 85,983 & 61,010 & 18,820 & - & 6,153 \\
\hline Jackson & 282,002 & 82,827 & 22,676 & 107,695 & 68,804 & 767,468 & 248,974 & 73,667 & 224,233 & 220,594 \\
\hline Jefferson & 386,552 & 79,107 & 41,315 & 157,951 & 108,179 & $1,294,155$ & 301,192 & 168,960 & 438,190 & 385,813 \\
\hline Lafayette & 206,356 & 75,648 & 37,394 & 44,067 & 49,247 & 535,347 & 195,078 & 100,784 & 65,639 & 173,846 \\
\hline Lake & 273,910 & 107,342 & 55,506 & 77,099 & 33,963 & 846,915 & 381,468 & 137,511 & 200,017 & 127,919 \\
\hline Lee & 47,715 & 17,724 & 29,262 & - & 729 & 67,357 & 26,010 & 41,347 & - & - \\
\hline Leon & 348,281 & 188,447 & 7,363 & 82,045 & 70,426 & $1,238,548$ & 725,702 & 16,337 & 253,651 & 242,858 \\
\hline Levy & 575,560 & 276,856 & 93,656 & 100,797 & 104,251 & $1,630,876$ & 746,028 & 271,726 & 251,223 & 361,899 \\
\hline Liberty & 570,902 & 260,589 & 93,194 & 161,665 & 55,454 & $1,905,941$ & 858,642 & 348,730 & 462,753 & 235,816 \\
\hline Madison & 268,875 & 75,245 & 49,479 & 101,565 & 42,586 & 731,843 & 192,993 & 160,342 & 236,254 & 142,254 \\
\hline Manatee & 30,081 & 3,166 & - & 14,058 & 12,857 & 117,819 & $14,4.17$ & - & 57,011 & 46,391 \\
\hline Marion & 554,956 & 336,764 & 21,978 & 79,971 & 116,243 & $1,692,959$ & 983,457 & 68,459 & 224,740 & 416,303 \\
\hline Martin & 31,501 & 28,276 & 2,347 & - & 878 & 107,767 & 104,390 & - & - & 3,377 \\
\hline Monroe & - & - & - & - & - & - & - & - & - & - \\
\hline Nassau & 396,025 & 187,438 & 38,005 & 123,437 & 47,145 & $1,017,394$ & 519,602 & 119,519 & 235,045 & 143,228 \\
\hline Okaloosa & 461,460 & 333,168 & 19,225 & 73,391 & 35,676 & $1,627,650$ & $1,281,224$ & 89,642 & 134,888 & 121,896 \\
\hline Okeechobee & 76,118 & 16,388 & 19,730 & 32,346 & 7,654 & 271,537 & 59,567 & 76,540 & 108,110 & 27,320 \\
\hline Orange & 201,530 & 53,081 & 79,265 & 55,812 & 13,372 & 647,545 & 221,014 & 221,056 & 155,403 & 50,072 \\
\hline Osceola & 305,989 & 50,490 & 161,140 & 63,094 & 31,265 & 975,157 & 246,341 & 486,602 & 168,344 & 73,870 \\
\hline Palm Beach & - & - & - & - & - & - & - & - & - & - \\
\hline Pasco & 193,575 & 12,646 & 81,115 & 45,348 & 54,466 & 599,559 & 39,216 & 222,145 & 140,632 & 197,566 \\
\hline Pinellas & 10,224 & 4,072 & 3,802 & 1,534 & 816 & 40,904 & 21,325 & 12,605 & 5,501 & 1,473 \\
\hline Polk & 317,686 & 80,258 & 110,413 & 91,670 & 35,345 & 994,378 & 304,280 & 313,382 & 242,402 & 134,314 \\
\hline Putnam & 304,588 & 172,368 & 11,535 & 76,682 & 44,003 & 811,329 & 421,372 & 40,763 & 219,233 & 129,961 \\
\hline Santa Rosa & 601,331 & 349,239 & 64,424 & 135,954 & 51,714 & $2,034,492$ & $1,301,478$ & 255,483 & 328,070 & 149,461 \\
\hline Sarasota & 25,588 & 16,884 & - & 3,765 & 4,939 & 89,649 & 55,087 & - & 11,750 & 22,812 \\
\hline Seminole & 77,271 & 30,927 & 1,238 & 12,236 & 32,870 & 316,940 & 132,458 & 5,627 & 46,711 & 132,144 \\
\hline St. Johns & 279,320 & 142,177 & 23,541 & 76,488 & 37,114 & 676,832 & 337,101 & 82,631 & 141,098 & 116,002 \\
\hline St. Lucie & 11,397 & 8,363 & 2,130 & 337 & 567 & 53,933 & 43,441 & 8,950 & 1,542 & - \\
\hline Sumter & 266,020 & 54,782 & 85,970 & 50,234 & 75,034 & 769,191 & 159,947 & 258,269 & 119,142 & 231.833 \\
\hline Suwannee & 220,560 & 101,993 & - & 28,712 & 89,855 & 705,620 & 263,984 & - & 102,934 & 338,702 \\
\hline Taylor & 399,108 & 150,826 & 75,298 & 98,472 & 74,512 & $1,017,314$ & 298,284 & 223,809 & 242,314 & 252,907 \\
\hline Union & 138,009 & 68,430 & 19,210 & 43,527 & 6,842 & 325,512 & 143,339 & 59,390 & 102,826 & 19,957 \\
\hline Volusia & 508,808 & 210,603 & 127,646 & 116,112 & 54,447 & $1,461,960$ & 702,904 & 286,510 & 276,964 & 195,582 \\
\hline Wakulla & 335,315 & 198,949 & 9,643 & 72,196 & 54,527 & $1,145,598$ & 733,811 & 36,271 & 195,435 & 180,081 \\
\hline Walton & 494,654 & 323,226 & 13,265 & 117,458 & 40,705 & $1,635,546$ & $1,127,003$ & 56,416 & 345,964 & 106,163 \\
\hline Washington & 259,917 & 87,908 & 33,049 & 87,136 & 51,824 & 628,665 & 222,867 & 141,345 & 121,768 & 142,685 \\
\hline Total & 366,431 & $6,975,095$ & $2,449,339$ & $3,590,661$ & $2,351,336$ & $45,287,230$ & $20,731,147$ & $7,580,392$ & $9,003,834$ & $7,971,857$ \\
\hline
\end{tabular}


Table 8-Average net annual growth of growing stock and sawtimber on timberland, by county and species group, Florida, $1987-1994$

\begin{tabular}{|c|c|c|c|c|c|c|c|c|c|c|}
\hline \multirow[b]{2}{*}{ County } & \multicolumn{5}{|c|}{ Growing stock } & \multicolumn{5}{|c|}{ Sawtimber } \\
\hline & $\begin{array}{c}\text { All } \\
\text { species } \\
\end{array}$ & Pine & $\begin{array}{c}\text { Other } \\
\text { softwood }\end{array}$ & $\begin{array}{c}\text { Soft } \\
\text { hardwood }\end{array}$ & $\begin{array}{c}\text { Hard } \\
\text { hardwood }\end{array}$ & $\begin{array}{c}\text { All } \\
\text { species }\end{array}$ & Pine & $\begin{array}{c}\text { Other } \\
\text { softwood }\end{array}$ & $\begin{array}{c}\text { Soft } \\
\text { hardwood }\end{array}$ & $\begin{array}{c}\text { Hard } \\
\text { hardwood }\end{array}$ \\
\hline & \multicolumn{5}{|c|}{ Thousand cubic feet } & \multicolumn{5}{|c|}{ Thousand board feet } \\
\hline Alachua & 23,261 & 17,827 & 537 & 1,334 & 3,563 & 67,261 & 44,340 & 2,954 & 5,257 & 14,710 \\
\hline Baker & 19,397 & 17,076 & 642 & 1,350 & 329 & 43,580 & 38,548 & 2,843 & 1,783 & 406 \\
\hline Bay & 18,094 & 16,843 & 65 & 491 & 695 & 17,533 & 14,304 & 328 & 1,333 & 1,568 \\
\hline Bradford & 7,596 & 6,298 & 240 & 524 & 534 & 16,046 & 11,978 & 915 & 1,194 & 1,959 \\
\hline Brevard & 2,986 & 2,027 & 163 & 210 & 586 & 12,953 & 8,755 & 657 & 1,226 & 2,315 \\
\hline Broward & - & - & - & - & - & - & - & - & - & - \\
\hline Calhoun & 17,332 & 15,018 & 240 & 1,475 & 599 & 37,386 & 25,877 & 1,713 & 6,248 & 3,548 \\
\hline Charlotte & 600 & 202 & 346 & 6 & 46 & 3,115 & 2,258 & 643 & - & 214 \\
\hline Citrus & 4,932 & 2,894 & 483 & 687 & 868 & 16,312 & 8,607 & 2,799 & 2,093 & 2,813 \\
\hline Clay & 16,286 & 12,456 & 168 & 2,087 & 1,575 & 48,000 & 36,808 & 842 & 4,342 & 6,008 \\
\hline Collier & 3,122 & 1,511 & 2,407 & -579 & -217 & 14,594 & 6,641 & 12,646 & $-2,692$ & $-2,001$ \\
\hline Columbia & 18,368 & 13,008 & 524 & 3,568 & 1,268 & 46,188 & 35,669 & 2,257 & 4,523 & 3,739 \\
\hline Dade & - & - & - & - & - & - & - & - & - & - \\
\hline De Soto & 1,185 & 27 & 104 & 696 & 358 & 5,476 & 563 & 593 & 3,524 & 796 \\
\hline Dixie & 26,298 & 16,970 & 2,362 & 3,274 & 3,692 & 75,600 & 42,969 & 9,109 & 8,192 & 15,330 \\
\hline Duval & 18,629 & 13,320 & 190 & 3,400 & 1,719 & 61,297 & 41,241 & 1,044 & 10,243 & 8,769 \\
\hline Escambia & 14,788 & 11,863 & 289 & 1,357 & 1,279 & 46,333 & 36,363 & 1,093 & 4,471 & 4,406 \\
\hline Flagler & 17,673 & 13,787 & 1,893 & 1,294 & 699 & 55,009 & 38,464 & 7,743 & 5,486 & 3,316 \\
\hline Franklin & 17,146 & 15,169 & 946 & 669 & 362 & 25,932 & 17,444 & 3,993 & 3,362 & 1,133 \\
\hline Gadsden & 16,405 & 10,169 & - & 3,137 & 3,099 & 41,880 & 16,198 & - & 12,642 & 13,040 \\
\hline Gilchrist & 9,610 & 8,349 & 164 & 280 & 817 & 17,120 & 13,988 & 894 & -226 & 2,464 \\
\hline Glades & 1,365 & 894 & 340 & 56 & 75 & 7,550 & 4,407 & 1,955 & 632 & 556 \\
\hline Gulf & 9,676 & 7,131 & 984 & 1,117 & 444 & 25,890 & 12,257 & 4,642 & 7,082 & 1,909 \\
\hline Hamilton & 14,591 & 11,169 & 290 & 1,489 & 1,643 & 23,195 & 13,588 & 1,293 & 2,149 & 6,165 \\
\hline Hardee & 3,218 & 690 & 958 & 491 & 1,079 & 11,100 & 4,108 & 2,731 & 728 & 3,533 \\
\hline Hendry & 1,890 & 301 & 1,664 & -118 & 43 & 9,046 & 1,849 & 7,294 & -519 & 422 \\
\hline Hernando & 4,803 & 3,363 & 157 & 445 & 838 & 15,512 & 10,768 & 561 & 1,505 & 2,678 \\
\hline Highlands & 1,074 & 177 & 524 & -17 & 390 & 6,461 & 1,315 & 3,104 & 201 & 1,841 \\
\hline Hillsborough & 4,831 & 644 & 1,823 & 969 & 1,395 & 22,310 & 3,337 & 9,153 & 5,298 & 4,522 \\
\hline Holmes & 10,514 & 6,908 & 165 & 1,832 & 1,609 & 27,138 & 18,108 & 728 & 4,263 & 4,039 \\
\hline Indian River & 270 & 189 & 41 & 11 & 29 & 1,286 & 952 & 198 & - & 136 \\
\hline Jackson & 14,326 & 7,403 & 913 & 2,743 & 3,267 & 43,867 & 20,529 & 2,776 & 7,306 & 13,256 \\
\hline Jefferson & 14,036 & 6,541 & 401 & 3,448 & 3,646 & 45,483 & 16,403 & 3,013 & 13,666 & 12,401 \\
\hline Lafayette & 13,043 & 9,776 & 355 & 1,505 & 1,407 & 31,597 & 24,981 & 2,109 & 1,297 & 3,210 \\
\hline Lake & 6,361 & 2,830 & 871 & 2,248 & 412 & 20,817 & 7,924 & 3,605 & 7,821 & 1,467 \\
\hline Lee & 1,267 & 1,084 & 101 & - & 82 & 4,011 & 3,062 & 949 & - & - \\
\hline Leon & 12,403 & 7,662 & 24 & 1,199 & 3,518 & 48,075 & 27,423 & 60 & 5,241 & 15,351 \\
\hline Levy & 27,646 & 20,522 & 1,859 & 2,176 & 3,089 & 84,793 & 59,613 & 8,114 & 5,640 & 11,426 \\
\hline Liberty & 16,034 & 10,823 & 822 & 3,078 & 1,311 & 47,453 & 26,004 & 3,657 & 11,514 & 6,278 \\
\hline Madison & 12,754 & 8,054 & 186 & 2,850 & 1,664 & 33,761 & 17,702 & 2,855 & 7,594 & 5,610 \\
\hline Manatee & 1,038 & 173 & - & 183 & 682 & 4,697 & 1,002 & - & 1,124 & 2,571 \\
\hline Marion & 24,848 & 18,984 & 170 & 1,671 & 4,023 & 83,759 & 60,357 & 1,109 & 7,980 & 14,313 \\
\hline Martin & 930 & 611 & 316 & - & 3 & 1,199 & 1,181 & - & - & 18 \\
\hline Monroe & - & - & - & - & - & - & - & - & - & - \\
\hline Nassau & 23,255 & 18,229 & 216 & 3,223 & 1,587 & 61,003 & 47,451 & 2,294 & 5,737 & 5,521 \\
\hline Okaloosa & 13,659 & 10,325 & 188 & 1,982 & 1,164 & 46,143 & 36,205 & 1,327 & 3,975 & 4,636 \\
\hline Okeechobee & 1,193 & 437 & 152 & 319 & 285 & 5,639 & 1,445 & 754 & 2,034 & 1,406 \\
\hline Orange & 5,688 & 2,332 & 1,801 & 937 & 618 & 25,875 & 13,021 & 6,401 & 3,057 & 3,396 \\
\hline Osceola & 5,561 & 1,370 & 2,904 & 782 & 505 & 28,208 & 7,645 & 14,697 & 3,501 & 2,365 \\
\hline Palm Beach & - & - & - & - & - & - & - & - & - & - \\
\hline Pasco & 2,814 & 1,114 & 797 & 200 & 703 & 13,124 & 3,143 & 4,894 & 1,793 & 3,294 \\
\hline Pinellas & 430 & 237 & 139 & 34 & 20 & 2,098 & 1,311 & 595 & 192 & - \\
\hline Polk & 10,114 & 3,263 & 3,323 & 2,426 & 1,102 & 41,112 & 10,926 & 13,773 & 9,801 & 6,612 \\
\hline Putnam & 13,014 & 11,250 & 149 & 835 & 780 & 39,217 & 34,071 & 963 & 971 & 3,212 \\
\hline Santa Rosa & 23,752 & 17,219 & 1,321 & 2,625 & 2,587 & 83,830 & 62,496 & 5,976 & 8,126 & 7,232 \\
\hline Sarasota & 1,161 & 846 & - & 34 & 281 & 4,287 & 3,391 & - & 323 & 573 \\
\hline Seminole & 2,179 & 1,259 & 24 & 413 & 483 & 12,192 & 6,423 & 144 & 2,146 & 3,479 \\
\hline St. Johns & 19,442 & 15,102 & 534 & 2,656 & 1,150 & 51,451 & 36,374 & 2,165 & 8,648 & 4,264 \\
\hline St. Lucie & 526 & 479 & 41 & - & 6 & 2,657 & 2,420 & 237 & - & - \\
\hline Sumter & 6,038 & 2,699 & 1,668 & 96 & 1,575 & 16,312 & 4,854 & 7,196 & -541 & 4,803 \\
\hline Suwannee & 11,990 & 8,625 & - & 382 & 2,983 & 27,699 & 16,754 & - & 1,271 & 9,674 \\
\hline Taylor & 28,996 & 22,639 & 1,423 & 2,309 & 2,625 & 62,638 & 37,584 & 6,399 & 6,945 & 11,710 \\
\hline Union & 6,714 & 5,514 & 443 & 541 & 216 & 16,203 & 11,818 & 1,595 & 1,796 & 994 \\
\hline Volusia & 16,864 & 9,920 & 2,067 & 3,693 & 1,184 & 49,534 & 25,276 & 8,687 & 9,364 & 6,207 \\
\hline Wakulla & 12,308 & 8,724 & 375 & 1,464 & 1,745 & 29,840 & 17,866 & 957 & 4,137 & 6,880 \\
\hline Walton & 22,806 & 18,253 & 262 & 2,380 & 1,911 & 66,321 & 50,668 & 1,228 & 9,328 & 5,097 \\
\hline Washington & 13,504 & 8,145 & 475 & 3,118 & 1,766 & 29,180 & 16,700 & 3,230 & 3,799 & 5,451 \\
\hline Total & 692,634 & 488,724 & 43,029 & 83,085 & 77,796 & $1,965,178$ & $1,225,727$ & 196,484 & 249,926 & 293,041 \\
\hline
\end{tabular}


Table 9-Average annual removals of growing stock and sawtimber on timberland, by county and species group, Florida, $1987-1994$

\begin{tabular}{|c|c|c|c|c|c|c|c|c|c|c|}
\hline \multirow[b]{2}{*}{ County } & \multicolumn{5}{|c|}{ Growing stock } & \multicolumn{5}{|c|}{ Sawtimber } \\
\hline & $\begin{array}{c}\text { All } \\
\text { species }\end{array}$ & Pine & $\begin{array}{c}\text { Other } \\
\text { softwood }\end{array}$ & $\begin{array}{c}\text { Soft } \\
\text { hardwood }\end{array}$ & $\begin{array}{c}\text { Hard } \\
\text { hardwood }\end{array}$ & $\begin{array}{c}\text { All } \\
\text { species }\end{array}$ & Pine & $\begin{array}{c}\text { Other } \\
\text { softwood }\end{array}$ & $\begin{array}{c}\text { Soft } \\
\text { hardwood }\end{array}$ & $\begin{array}{c}\text { Hard } \\
\text { hardwood }\end{array}$ \\
\hline & \multicolumn{5}{|c|}{ Thousand cubic feet } & \multicolumn{5}{|c|}{ Thousand board feet } \\
\hline Alachua & 18,281 & 14,534 & 741 & 634 & 2,372 & 49,275 & 34,149 & 3,187 & 2,344 & 9,595 \\
\hline Baker & 19,337 & 18,581 & 694 & 28 & 34 & 42,600 & 41,604 & 996 & - & - \\
\hline Bay & 18,143 & 18,088 & - & - & 55 & 24,401 & 24,401 & - & - & - \\
\hline Bradford & 3,338 & 3,189 & 68 & - & 81 & 4,656 & 4,241 & - & - & 415 \\
\hline Brevard & 3,232 & 2,071 & 1,161 & - & - & 9,014 & 5,664 & 3,350 & - & - \\
\hline Broward & - & - & - & - & - & - & - & - & - & - \\
\hline Calhoun & 15,230 & 14,332 & - & 322 & 576 & 27,959 & 25,656 & - & 940 & 1,363 \\
\hline Charlotte & 99 & - & - & 35 & 64 & 366 & - & - & - & 366 \\
\hline Citrus & 4,424 & 4,128 & 181 & - & 115 & 10,747 & 10,187 & 560 & - & - \\
\hline Clay & 13,134 & 11,760 & 113 & 775 & 486 & 36,356 & 33,406 & 387 & 1,393 & 1,170 \\
\hline Collier & 5,238 & 434 & 3,896 & 454 & 454 & 17,605 & 2,018 & 11,994 & 2,191 & 1,402 \\
\hline Columbia & 16,444 & 14,141 & 620 & 1,304 & 379 & 48,924 & 43,334 & 2,197 & 2,311 & 1,082 \\
\hline Dade & - & - & - & - & - & - & - & - & - & - \\
\hline De Soto & 720 & 439 & 44 & 40 & 197 & 3,516 & 2,369 & 281 & - & 866 \\
\hline Dixie & 23,659 & 13,769 & 4,001 & 3,134 & 2,755 & 67,665 & 36,780 & 12,056 & 11,389 & 7,440 \\
\hline Duval & 8,979 & 5,099 & 90 & 1,749 & 2,041 & 28,252 & 16,518 & - & 4,926 & 6,808 \\
\hline Escambia & 13,818 & 10,139 & - & 2,420 & 1,259 & 42,638 & 33,337 & - & 6,334 & 2,967 \\
\hline Flagler & 15,892 & 11,816 & 3,705 & 371 & - & 29,202 & 18,644 & 9,974 & 584 & - \\
\hline Franklin & 6,591 & 6,591 & - & - & - & 8,509 & 8,509 & - & - & - \\
\hline Gadsden & 11,277 & 7,383 & - & 2,460 & 1,434 & 36,888 & 20,377 & - & 9,794 & 6,717 \\
\hline Gilchrist & 4,885 & 4,885 & - & - & - & 12,954 & 12,954 & - & - & - \\
\hline Glades & 3,528 & 3,434 & 94 & - & - & 10,909 & 10,508 & 401 & - & - \\
\hline Gulf & 4,864 & 4,864 & - & - & - & 6,518 & 6,518 & - & - & - \\
\hline Hamilton & 13,609 & 10,499 & 1,080 & 1,680 & 350 & 28,198 & 21,342 & 2,790 & 3,288 & 778 \\
\hline Hardee & 143 & 143 & - & - & - & - & - & - & - & - \\
\hline Hendry & 3,275 & 2,727 & 495 & - & 53 & 9,711 & 8,931 & 780 & - & - \\
\hline Hernando & 2,420 & 1,120 & - & 795 & 505 & 10,047 & 5,598 & - & 2,474 & 1,975 \\
\hline Highlands & 1,080 & 289 & - & 506 & 285 & 4,233 & 1,204 & - & 1,438 & 1,591 \\
\hline Hillsborough & 3,473 & 328 & 1,181 & 544 & 1,420 & 14,945 & 1,920 & 6,115 & 2,002 & 4,908 \\
\hline Holmes & 6,276 & 5,741 & - & - & 535 & 24,339 & 22,496 & - & - & 1,843 \\
\hline Indian River & 180 & 94 & - & - & 86 & 1,047 & 607 & - & - & 440 \\
\hline Jackson & 22,731 & 13,400 & 195 & 3,352 & 5,784 & 87,541 & 52,835 & 1,082 & 11,048 & 22,576 \\
\hline Jefferson & 9,053 & 7,518 & 106 & 323 & 1,106 & 36,251 & 32,401 & 195 & 1,150 & 2,505 \\
\hline Lafayette & 17,321 & 15,214 & 1,209 & 490 & 408 & 36,884 & 31,408 & 2,663 & 860 & 1,953 \\
\hline Lake & 5,759 & 1,770 & 2,841 & 920 & 228 & 18,909 & 8,785 & 8,258 & 998 & 868 \\
\hline Lee & 810 & 810 & - & - & - & 1,552 & 1,552 & - & - & - \\
\hline Leon & 10,518 & 5,683 & - & 1,528 & 3,307 & 39,492 & 23,980 & - & 3,096 & 12,416 \\
\hline Levy & 17,889 & 13,612 & 1,328 & 868 & 2,081 & 45,890 & 36,147 & 2,862 & 1,752 & 5,129 \\
\hline Liberty & 12,319 & 9,569 & 162 & 957 & 1,631 & 31,470 & 22,807 & 221 & 1,881 & 6,561 \\
\hline Madison & 17,010 & 11,173 & 1,526 & 3,630 & 681 & 47,294 & 32,418 & 5,599 & 8,697 & 580 \\
\hline Manatee & 815 & 720 & - & - & 95 & 3,720 & 3,488 & - & - & 232 \\
\hline Marion & 20,506 & 20,181 & - & 33 & 292 & 47,005 & 46,522 & - & - & 483 \\
\hline Martin & - & - & - & - & - & - & - & - & - & - \\
\hline Monroe & - & - & - & - & - & - & - & - & - & - \\
\hline Nassau & 19,957 & 18,085 & 118 & 564 & 1,190 & 52,340 & 48,400 & 305 & 219 & 3,416 \\
\hline Okaloosa & 8,576 & 8,495 & - & 44 & 37 & 23,735 & 23,735 & - & - & - \\
\hline Okeechobee & 458 & 458 & - & - & - & 1,157 & 1,157 & - & - & - \\
\hline Orange & 6,123 & 2,425 & 819 & 1,526 & 1,353 & 15,378 & 4,621 & 1,792 & 3,530 & 5,435 \\
\hline Osceola & 3,051 & 645 & 2,342 & 64 & - & 8,062 & 2,498 & 5,343 & 221 & - \\
\hline Palm Beach & - & - & - & - & - & - & - & - & - & - \\
\hline Pasco & 5,342 & 3,320 & 1,611 & 113 & 298 & 16,622 & 12,121 & 3,375 & - & 1,126 \\
\hline Pinellas & 752 & 752 & - & - & - & 2,871 & 2,871 & - & - & - \\
\hline Polk & 6,451 & 104 & 5,462 & 405 & 480 & 14,970 & - & 11,854 & 273 & 2,843 \\
\hline Putnam & 12,494 & 9,927 & 72 & 1,851 & 644 & 36,210 & 27,607 & 329 & 5,583 & 2,691 \\
\hline Santa Rosa & 19,964 & 18,719 & - & 149 & 1,096 & 49,366 & 47,023 & - & 521 & 1,822 \\
\hline Sarasota & 988 & 775 & - & - & 213 & 2,894 & 2,894 & - & - & - \\
\hline Seminole & 2,332 & 426 & - & 791 & 1,115 & 10,263 & 2,066 & - & 3,547 & 4,650 \\
\hline St. Johns & 16,599 & 13,060 & 1,084 & 1,380 & 1,075 & 42,662 & 33,226 & 2,138 & 3,525 & 3,773 \\
\hline St. Lucie & 1,613 & 1,613 & - & - & - & 5,219 & 5,219 & - & - & - \\
\hline Sumter & 1,145 & 730 & - & - & 415 & 3,632 & 2,017 & - & - & 1,615 \\
\hline Suwannee & 3,525 & 2,368 & - & 67 & 1,090 & 6,758 & 4,527 & - & 282 & 1,949 \\
\hline Taylor & 33,044 & 24,585 & 1,569 & 2,717 & 4,173 & 72,363 & 46,805 & 5,931 & 7,566 & 12,061 \\
\hline Union & 3,713 & 3,469 & 114 & 130 & - & 8,799 & 8,155 & 452 & 192 & - \\
\hline Volusia & 12,153 & 9,214 & 2,414 & 478 & 47 & 32,666 & 25,198 & 6,874 & 594 & - \\
\hline Wakulla & 8,711 & 6,023 & - & 703 & 1,985 & 30,135 & 21,460 & - & 2,641 & 6,034 \\
\hline Walton & 11,933 & 11,585 & - & 34 & 314 & 24,481 & 24,481 & - & - & - \\
\hline Washington & 5,495 & 4,390 & 61 & 470 & 574 & 14,869 & 11,858 & - & 1,571 & 1,440 \\
\hline Total & 560,719 & 431,436 & 41,197 & 40,838 & 47,248 & $1,482,934$ & $1,103,554$ & 114,341 & 111,155 & 153,884 \\
\hline
\end{tabular}


Table 10-Area of timberland, by forest type and ownership class, Florida, 1995

\begin{tabular}{|c|c|c|c|c|c|c|}
\hline \multirow[b]{2}{*}{ Forest type } & \multirow[b]{2}{*}{$\begin{array}{c}\text { All } \\
\text { ownerships }\end{array}$} & \multicolumn{5}{|c|}{ Ownership class } \\
\hline & & $\begin{array}{c}\text { National } \\
\text { forest }\end{array}$ & $\begin{array}{l}\text { Other } \\
\text { public }\end{array}$ & \multirow[t]{2}{*}{$\begin{array}{l}\text { Forest } \\
\text { industry }\end{array}$} & \multirow[t]{2}{*}{$\begin{array}{c}\text { Forest } \\
\text { industry- } \\
\text { leased }\end{array}$} & \multirow[t]{2}{*}{$\begin{array}{l}\text { Other } \\
\text { private }\end{array}$} \\
\hline & & & Acre & & & \\
\hline \multicolumn{7}{|l|}{ Softwood types } \\
\hline Longleaf pine & 740,488 & 175,807 & 225,376 & 72,716 & - & 266,589 \\
\hline Slash pine & $5,130,856$ & 328,591 & 425,342 & $1,994,134$ & 347,153 & $2,035,636$ \\
\hline Loblolly pine & 807,284 & 7,138 & 23,893 & 298,972 & 22,408 & 454,873 \\
\hline Shortleaf pine & 16,908 & - & - & 9,098 & - & 7,810 \\
\hline Virginia pine & - & - & - & - & - & - \\
\hline Sand pine & 633,766 & 187,567 & 95,899 & 162,794 & - & 187,506 \\
\hline Eastern redcedar & - & - & - & - & - & - \\
\hline Pond pine & 108,486 & 26,351 & 7,861 & 14,621 & - & 59,653 \\
\hline Spruce pine & - & - & - & - & - & - \\
\hline Pitch pine & - & - & - & - & - & - \\
\hline Table Mountain pine & - & - & - & - & - & 二 \\
\hline Total & $7,437,788$ & 725,454 & 778,371 & $2,552,335$ & 369,561 & $3,012,067$ \\
\hline \multicolumn{7}{|l|}{ Hardwood types } \\
\hline Oak-pine & $1,478,980$ & 82,906 & 222,650 & 295,567 & 45,383 & 832,474 \\
\hline Oak-hickory & $1,437,863$ & 14,213 & 106,046 & 160,172 & 32,462 & $1,124,970$ \\
\hline Chestnut oak & - & - & - & - & - & - \\
\hline Southern scrub oak & 575,480 & 33,809 & 100,641 & 29,822 & 7,851 & 403,357 \\
\hline Oak-gum-cypress & $3,678,526$ & 173,140 & 589,344 & 960,800 & 130,720 & $1,824,522$ \\
\hline Elm-ash-cottonwood & 42,023 & - & 5,198 & 16,810 & - & 20,015 \\
\hline Total & $7,212,872$ & 304,068 & $1,023,879$ & $1,463,171$ & 216,416 & $4,205,338$ \\
\hline All types & $14,650,660$ & $1,029,522$ & $1,802,250$ & $4,015,506$ & 585,977 & $7,217,405$ \\
\hline
\end{tabular}

Table 11-Area of timberland, by ownership and stocking classes of growing-stock trees, Florida, 1995

\begin{tabular}{|c|c|c|c|c|c|c|}
\hline \multirow[b]{2}{*}{ Ownership class } & \multirow{2}{*}{$\begin{array}{c}\text { All } \\
\text { classes }\end{array}$} & \multicolumn{5}{|c|}{ Stocking class (percent) ${ }^{a}$} \\
\hline & & $>130$ & $100-130$ & $60-99$ & 16.7-59 & $<16.7$ \\
\hline & \multicolumn{6}{|c|}{ Acres } \\
\hline National forest & $1,029,522$ & 26,794 & 228,474 & 416,224 & 282,641 & 75,389 \\
\hline Other public & $1,802,250$ & 130,150 & 291,388 & 641,611 & 562,143 & 176,958 \\
\hline Forest industry & $4,015,506$ & 254,178 & $1,040,302$ & $1,654,515$ & 806,110 & 260,401 \\
\hline Forest industry-leased & 585,977 & 27,398 & 222,462 & 248,026 & 57,215 & 30,876 \\
\hline Other private & $7,217,405$ & 310,791 & $1,548,144$ & $2,586,793$ & $1,961,961$ & 809,716 \\
\hline All ownerships & $14,650,660$ & 749,311 & $3,330,770$ & $5,547,169$ & $3,670,070$ & $1,353,340$ \\
\hline
\end{tabular}

"See stocking standards under "stocking" in definitions. 
Table 12-Area of timberland, by forest type and stand-size class, Florida, 1995

\begin{tabular}{|c|c|c|c|c|c|}
\hline \multirow[b]{2}{*}{ Forest type } & \multirow[b]{2}{*}{$\begin{array}{c}\text { All } \\
\text { stands }\end{array}$} & \multicolumn{3}{|c|}{ Stand-size class } & \multirow[b]{2}{*}{$\begin{array}{c}\text { Nonstocked } \\
\text { areas }\end{array}$} \\
\hline & & Sawtimber & Poletimber & $\begin{array}{l}\text { Sapling- } \\
\text { seedling }\end{array}$ & \\
\hline & \multicolumn{5}{|c|}{ Acres } \\
\hline \multicolumn{6}{|l|}{ Softwood types } \\
\hline Longleaf pine & 740,488 & 402,669 & 77,826 & 234,866 & 25,127 \\
\hline Slash pine & $5,130,856$ & 929,449 & $1,895,627$ & $2,080,133$ & 225,647 \\
\hline Loblolly pine & 807,284 & 164,767 & 223,559 & 418,958 & - \\
\hline Shortleaf pine & 16,908 & 7,810 & 2,693 & 6,405 & - \\
\hline Virginia pine & - & - & - & - & - \\
\hline Sand pine & 633,766 & 95,113 & 205,217 & 323,836 & 9,600 \\
\hline Eastern redcedar & - & - & - & - & - \\
\hline Pond pine & 108,486 & 54,898 & 32,290 & 18,326 & 2,972 \\
\hline Spruce pine & - & - & - & - & - \\
\hline Pitch pine & - & - & - & - & - \\
\hline Table Mountain pine & - & - & - & - & - \\
\hline Total & $7,437,788$ & $1,654,706$ & $2,437,212$ & $3,082,524$ & 263,346 \\
\hline \multicolumn{6}{|l|}{ Hardwood types } \\
\hline Oak-pine & $1,478,980$ & 457,015 & 267,811 & 738,455 & 15,699 \\
\hline Oak-hickory & $1,437,863$ & 631,019 & 287,550 & 497,081 & 22,213 \\
\hline Chestnut oak & - & - & - & - & - \\
\hline Southern scrub oak & 575,480 & 20,384 & 62,311 & 482,600 & 10,185 \\
\hline Oak-gum-cypress & $3,678,526$ & $2,012,853$ & 957,186 & 592,278 & 116,209 \\
\hline Elm-ash-cottonwood & 42,023 & 10,703 & 11,976 & 19,344 & - \\
\hline Total & $7,212,872$ & $3,131,974$ & $1,586,834$ & $2,329,758$ & 164,306 \\
\hline All types & $14,650,660$ & $4,786,680$ & $4,024,046$ & $5,412,282$ & 427,652 \\
\hline
\end{tabular}

Table 13-Area of timberland, by stand-age and broad management classes, all ownerships, Florida, 1995

\begin{tabular}{|c|c|c|c|c|c|c|}
\hline \multirow[b]{2}{*}{$\begin{array}{l}\text { Stand-age class } \\
\text { (years) }\end{array}$} & \multirow[b]{2}{*}{$\begin{array}{c}\text { All } \\
\text { classes } \\
\end{array}$} & \multicolumn{5}{|c|}{ Broad management class } \\
\hline & & $\begin{array}{c}\text { Pine } \\
\text { plantation }\end{array}$ & $\begin{array}{l}\text { Natural } \\
\text { pine }\end{array}$ & Oak-pine & $\begin{array}{c}\text { Upland } \\
\text { hardwood }\end{array}$ & $\begin{array}{l}\text { Lowland } \\
\text { hardwood }\end{array}$ \\
\hline & \multicolumn{6}{|c|}{ Acres } \\
\hline $0-10$ & $2,935,883$ & $2,059,867$ & 257,700 & 277,020 & 206,784 & 134,512 \\
\hline $11-20$ & $1,980,683$ & $1,404,645$ & 241,655 & 132,090 & 112,707 & 89,586 \\
\hline $21-30$ & $1,248,987$ & 806,973 & 191,965 & 55,825 & 72,706 & 121,518 \\
\hline $31-40$ & 807,063 & 255,791 & 275,386 & 61,062 & 36,314 & 178,510 \\
\hline $41-50$ & 915,265 & 24,951 & 373,664 & 100,680 & 80,610 & 335,360 \\
\hline $51-60$ & 890,426 & 6,855 & 291,587 & 96,744 & 85,138 & 410,102 \\
\hline $61-70$ & 736,372 & - & 189,280 & 55,820 & 53,989 & 437,283 \\
\hline $71-80$ & 482,820 & 2,579 & 56,507 & 26,390 & 42,259 & 355,085 \\
\hline $81+$ & 681,444 & - & 43,087 & 55,638 & 73,707 & 509,012 \\
\hline No manageable stand & $3,971,717$ & 72,066 & 883,230 & 617,711 & $1,249,129$ & $1,149,581$ \\
\hline All classes & $14,650,660$ & $4,633,727$ & $2,804,061$ & $1,478,980$ & $2,013,343$ & $3,720,549$ \\
\hline
\end{tabular}


Table 14-Area of timberland, by stand-age and broad management classes, public ownerships, Florida, 1995

\begin{tabular}{|c|c|c|c|c|c|c|}
\hline \multirow[b]{2}{*}{$\begin{array}{c}\text { Stand-age class } \\
\text { (years) }\end{array}$} & \multirow[b]{2}{*}{$\begin{array}{c}\text { All } \\
\text { classes }\end{array}$} & \multicolumn{5}{|c|}{ Broad management class } \\
\hline & & $\begin{array}{c}\text { Pine } \\
\text { plantation }\end{array}$ & $\begin{array}{l}\text { Natural } \\
\text { pine }\end{array}$ & Oak-pine & $\begin{array}{c}\text { Upland } \\
\text { hardwood }\end{array}$ & $\begin{array}{l}\text { Lowland } \\
\text { hardwood }\end{array}$ \\
\hline & \multicolumn{6}{|c|}{ Acres } \\
\hline $0-10$ & 253,516 & 132,960 & 52,812 & 39,965 & 9,176 & 18,603 \\
\hline $11-20$ & 245,796 & 169,421 & 44,701 & 11,179 & 16,216 & 4,279 \\
\hline $21-30$ & 200,162 & 137,514 & 36,071 & 9,429 & 3,190 & 13,958 \\
\hline $31-40$ & 167,340 & 45,625 & 88,093 & 7,513 & - & 26,109 \\
\hline $41-50$ & 212,503 & 14,905 & 137,516 & 17,334 & 4,249 & 38,499 \\
\hline $51-60$ & 271,837 & 2,064 & 172,139 & 16,644 & - & 80,990 \\
\hline $61-70$ & 277,144 & - & 130,738 & 17,282 & 2,730 & 126,394 \\
\hline $71-80$ & 143,706 & - & 41,166 & 2,669 & - & 99,871 \\
\hline $81+$ & 215,462 & - & 37,888 & 10,436 & 10,118 & 157,020 \\
\hline No manageable stand & 844,306 & 18,961 & 241,251 & 173,105 & 209,030 & 201,959 \\
\hline All classes & $2,831,772$ & 521,450 & 982,375 & 305,556 & 254,709 & 767,682 \\
\hline
\end{tabular}

Table 15-Area of timberland, by stand-age and broad management classes, forest industry, ${ }^{a}$ Florida, 1995

\begin{tabular}{|c|c|c|c|c|c|c|}
\hline \multirow[b]{2}{*}{$\begin{array}{c}\text { Stand-age class } \\
\text { (years) }\end{array}$} & \multirow[b]{2}{*}{$\begin{array}{c}\text { All } \\
\text { classes }\end{array}$} & \multicolumn{5}{|c|}{ Broad management class } \\
\hline & & $\begin{array}{c}\text { Pine } \\
\text { plantation }\end{array}$ & $\begin{array}{l}\text { Natural } \\
\text { pine }\end{array}$ & Oak-pine & $\begin{array}{c}\text { Upland } \\
\text { hardwood }\end{array}$ & $\begin{array}{l}\text { Lowland } \\
\text { hardwood }\end{array}$ \\
\hline & \multicolumn{6}{|c|}{ Acres } \\
\hline $0-10$ & $1,321,432$ & $1,100,272$ & 44,172 & 83,647 & 42,138 & 51,203 \\
\hline $11-20$ & 946,806 & 794,074 & 36,012 & 57,351 & 14,534 & 44,835 \\
\hline $21-30$ & 521,349 & 449,095 & 19,275 & 13,542 & 4,707 & 34,730 \\
\hline $31-40$ & 201,485 & 102,844 & 33,848 & 10,914 & 5,672 & 48,207 \\
\hline $41-50$ & 220,222 & 5,552 & 71,027 & 22,022 & 8,390 & 113,231 \\
\hline $51-60$ & 195,424 & 2,393 & 30,185 & 36,277 & 2,641 & 123,928 \\
\hline $61-70$ & 169,019 & - & 27,332 & 11,087 & 14,250 & 116,350 \\
\hline $71-80$ & 98,376 & 2,579 & 5,376 & 3,203 & 10,649 & 76,569 \\
\hline $81+$ & 133,147 & - & 2,579 & 14,654 & 3,031 & 112,883 \\
\hline No manageable stand & 794,223 & 25,193 & 170,088 & 88,253 & 124,295 & 386,394 \\
\hline All classes & $4,601,483$ & $2,482,002$ & 439,894 & 340,950 & 230,307 & $1,108,330$ \\
\hline
\end{tabular}

${ }^{\circ}$ Includes 585,977 acres of other private land under long-term lease. 
Table 16-Area of timberland, by stand-age and broad management classes, other private ownerships, ${ }^{a}$ Florida, 1995

\begin{tabular}{|c|c|c|c|c|c|c|}
\hline \multirow[b]{2}{*}{$\begin{array}{l}\text { Stand-age class } \\
\text { (years) }\end{array}$} & \multirow[b]{2}{*}{$\begin{array}{c}\text { All } \\
\text { classes }\end{array}$} & \multicolumn{5}{|c|}{ Broad management class } \\
\hline & & $\begin{array}{c}\text { Pine } \\
\text { plantation }\end{array}$ & $\begin{array}{l}\text { Natural } \\
\text { pine }\end{array}$ & Oak-pine & $\begin{array}{c}\text { Upland } \\
\text { hardwood }\end{array}$ & $\begin{array}{l}\text { Lowland } \\
\text { hardwood }\end{array}$ \\
\hline & \multicolumn{6}{|c|}{ Acres } \\
\hline $0-10$ & $1,360,935$ & 826,635 & 160,716 & 153,408 & 155,470 & 64,706 \\
\hline $11-20$ & 788,081 & 441,150 & 160,942 & 63,560 & 81,957 & 40,472 \\
\hline $21-30$ & 527,476 & 220,364 & 136,619 & 32,854 & 64,809 & 72,830 \\
\hline $31-40$ & 438,238 & 107,322 & 153,445 & 42,635 & 30,642 & 104,194 \\
\hline $41-50$ & 482,540 & 4,494 & 165,121 & 61,324 & 67,971 & 183,630 \\
\hline $51-60$ & 423,165 & 2,398 & 89,263 & 43,823 & 82,497 & 205,184 \\
\hline $61-70$ & 290,209 & - & 31,210 & 27,451 & 37,009 & 194,539 \\
\hline $71-80$ & 240,738 & - & 9,965 & 20,518 & 31,610 & 178,645 \\
\hline $81+$ & 332,835 & - & 2,620 & 30,548 & 60,558 & 239,109 \\
\hline No manageable stand & $2,333,188$ & 27,912 & 471,891 & 356,353 & 915,804 & 561,228 \\
\hline All classes & $7,217,405$ & $1,630,275$ & $1,381,792$ & 832,474 & $1,528,327$ & $1,844,537$ \\
\hline
\end{tabular}

${ }^{a}$ Excludes 585,977 acres of other private land under long-term lease to forest industry.

Table 17-Area of timberland, by broad management and stand-volume classes, Florida, 1995

\begin{tabular}{|c|c|c|c|c|c|c|}
\hline \multirow[b]{2}{*}{ Broad management class } & \multirow[b]{2}{*}{$\begin{array}{c}\text { All } \\
\text { classes }\end{array}$} & \multicolumn{5}{|c|}{$\begin{array}{c}\text { Stand-volume class } \\
\text { (cubic feet of growing stock per acre) }\end{array}$} \\
\hline & & $0-499$ & $500-999$ & $1000-1499$ & $1500-1999$ & $2000+$ \\
\hline & \multicolumn{6}{|c|}{ Acres } \\
\hline Pine plantation & $4,633,727$ & $2,695,632$ & 734,026 & 562,283 & 331,840 & 309,946 \\
\hline Natural pine & $2,804,061$ & $1,023,368$ & 515,306 & 399,307 & 315,096 & 550,984 \\
\hline Oak-pine & $1,478,980$ & 763,373 & 275,523 & 157,034 & 67,486 & 215,564 \\
\hline Upland hardwood & $2,013,343$ & $1,222,042$ & 301,239 & 156,585 & 149,588 & 183,889 \\
\hline Lowland hardwood & $3,720,549$ & 914,599 & 477,120 & 459,799 & 448,890 & $1,420,141$ \\
\hline All classes & $14,650,660$ & $6,619,014$ & $2,303,214$ & $1,735,008$ & $1,312,900$ & $2,680,524$ \\
\hline
\end{tabular}


Table 18-Volume of growing stock on timberland, by broad management class, species group, and stand-age class, Florida, 1995

\begin{tabular}{|c|c|c|c|c|c|c|c|c|c|c|c|}
\hline \multirow{2}{*}{$\begin{array}{l}\text { Broad management } \\
\text { class and } \\
\text { species group }\end{array}$} & \multirow{2}{*}{$\begin{array}{c}\text { All } \\
\text { classes }\end{array}$} & \multirow{2}{*}{$\begin{array}{c}\text { No } \\
\text { manageable } \\
\text { stand }\end{array}$} & \multicolumn{9}{|c|}{ Stand-age class (years) } \\
\hline & & & $0-10$ & $11-20$ & $21-30$ & $31-40$ & $41-50$ & $51-60$ & $61-70$ & $71-80$ & $81+$ \\
\hline & \multicolumn{11}{|c|}{ Thousand cubic feet } \\
\hline \multicolumn{12}{|l|}{ Pine plantation } \\
\hline Softwood & $2,743,583$ & 17,555 & 132,993 & $1,009,979$ & $1,121,288$ & 399,674 & 40,033 & 15,083 & - & 6,978 & - \\
\hline Hardwood & 51,984 & 517 & 6,035 & 17,512 & 21,512 & 6,160 & - & 248 & - & - & - \\
\hline Total & $2,795,567$ & 18,072 & 139,028 & $1,027,491$ & $1,142,800$ & 405,834 & 40,033 & 15,331 & - & 6,978 & - \\
\hline \multicolumn{12}{|l|}{ Natural pine } \\
\hline Softwood & $3,082,014$ & 322,984 & 64,452 & 136,270 & 199,199 & 439,668 & 682,264 & 606,349 & 393,768 & 125,562 & 111,498 \\
\hline Hardwood & 216,390 & 9,821 & 8,778 & 11,192 & 11,536 & 22,322 & 47,211 & 55,647 & 32,668 & 3,443 & 13,772 \\
\hline Total & $3,298,404$ & 332,805 & 73,230 & 147,462 & 210,735 & 461,990 & 729,475 & 661,996 & 426,436 & 129,005 & 125,270 \\
\hline \multicolumn{12}{|l|}{ Oak-pine } \\
\hline Softwood & 867,418 & 208,671 & 34,098 & 39,695 & 33,702 & 48,172 & 111,338 & 160,284 & 83,326 & 40,908 & 107,224 \\
\hline Hardwood & 480,032 & 46,035 & 17,573 & 16,767 & 17,912 & 29,303 & 81,632 & 114,853 & 62,927 & 32,524 & 60,506 \\
\hline Total & $1,347,450$ & 254,706 & 51,671 & 56,462 & 51,614 & 77,475 & 192,970 & 275,137 & 146,253 & 73,432 & 167,730 \\
\hline \multicolumn{12}{|l|}{ Upland hardwood } \\
\hline Softwood & 156,993 & 73,159 & 11,803 & 12,084 & 14,052 & 8,360 & 10,542 & 14,342 & 4,003 & 4,923 & 3,725 \\
\hline Hardwood & $1,100,456$ & 259,495 & 45,006 & 41,625 & 65,481 & 46,659 & 134,938 & 162,805 & 99,811 & 79,856 & 164,780 \\
\hline Total & $1,257,449$ & 332,654 & 56,809 & 53,709 & 79,533 & 55,019 & 145,480 & 177,147 & 103,814 & 84,779 & 168,505 \\
\hline \multicolumn{12}{|l|}{ Lowland hardwood } \\
\hline Softwood & $2,574,426$ & 152,372 & 17,231 & 8,463 & 44,664 & 103,403 & 173,378 & 317,243 & 468,257 & 432,056 & 857,359 \\
\hline Hardwood & $4,093,135$ & 375,763 & 23,694 & 27,507 & 73,147 & 164,300 & 465,337 & 712,394 & 741,543 & 651,578 & 857,872 \\
\hline Total & $6,667,561$ & 528,135 & 40,925 & 35,970 & 117,811 & 267,703 & 638,715 & $1,029,637$ & $1,209,800$ & $1,083,634$ & $1,715,231$ \\
\hline \multicolumn{12}{|l|}{ All types } \\
\hline Softwood & $9,424,434$ & 774,741 & 260,577 & $1,206,491$ & $1,412,905$ & 999,277 & $1,017,555$ & $1,113,301$ & 949,354 & 610,427 & $1,079,806$ \\
\hline Hardwood & $5,941,997$ & 691,631 & 101,086 & 114,603 & 189,588 & 268,744 & 729,118 & $1,045,947$ & 936,949 & 767,401 & $1,096,930$ \\
\hline Total & $15,366,431$ & $1,466,372$ & 361,663 & $1,321,094$ & $1,602,493$ & $1,268,021$ & $1,746,673$ & $2,159,248$ & $1,886,303$ & $1,377,828$ & $2,176,736$ \\
\hline
\end{tabular}


Table 19-Average net annual growth of growing stock on timberland, by broad management class, species group, and stand-age class, Florida, 1987-1994

\begin{tabular}{|c|c|c|c|c|c|c|c|c|c|c|c|}
\hline \multirow{2}{*}{$\begin{array}{l}\text { Broad management } \\
\text { class }^{a} \text { and } \\
\text { species group }\end{array}$} & \multirow{2}{*}{$\begin{array}{c}\text { All } \\
\text { classes }\end{array}$} & \multirow{2}{*}{$\begin{array}{c}\text { No } \\
\text { manageable } \\
\text { stand }\end{array}$} & \multicolumn{9}{|c|}{ Stand-age class (years) ${ }^{a}$} \\
\hline & & & $0-10$ & $11-20$ & $21-30$ & $31-40$ & $41-50$ & $51-60$ & $61-70$ & $71-80$ & $81+$ \\
\hline & \multicolumn{11}{|c|}{ Thousand cubic feet } \\
\hline Hardwood & 5,105 & 22 & 734 & 1,855 & 2,194 & 263 & 37 & - & - & - & - \\
\hline Total & 333,095 & 4,320 & 113,511 & 132,699 & 72,212 & 8,946 & 1,100 & 211 & 96 & - & - \\
\hline Total & 126,246 & 19,833 & 8,208 & 14,380 & 21,253 & 27,807 & 20,028 & 10,167 & 1,959 & 2,104 & 507 \\
\hline \multicolumn{12}{|l|}{ Oak-pine } \\
\hline Softwood & 27,886 & 7,241 & 4,535 & 4,198 & 1,606 & 2,662 & 4,713 & 1,596 & 491 & 508 & 336 \\
\hline Hardwood & 15,193 & 2,841 & 963 & 1,073 & 1,175 & 2,851 & 3,650 & 994 & 1,020 & 248 & 378 \\
\hline Total & 43,079 & 10,082 & 5,498 & 5,271 & 2,781 & 5,513 & 8,363 & 2,590 & 1,511 & 756 & 714 \\
\hline \multicolumn{12}{|l|}{ Lowland hardwood } \\
\hline Softwood & 53,248 & 5,989 & 1,375 & 1,240 & 2,516 & 3,636 & 6,271 & 10,099 & 7,818 & 5,972 & 8,332 \\
\hline Hardwood & 94,881 & 16,136 & 2,346 & 2,942 & 5,203 & 12,020 & 15,707 & 13,990 & 11,065 & 6,711 & 8,761 \\
\hline Total & 148,129 & 22,125 & 3,721 & 4,182 & 7,719 & 15,656 & 21,978 & 24,089 & 18,883 & 12,683 & 17,093 \\
\hline \multicolumn{12}{|l|}{ All types } \\
\hline Softwood & 531,753 & 40,143 & 127,512 & 150,683 & 94,994 & 40,942 & 28,966 & 21,209 & 10,047 & 8,185 & 9,072 \\
\hline Hardwood & 160,881 & 32,252 & 6,405 & 10,682 & 11,895 & 20,340 & 26,712 & 19,253 & 14,575 & 7,874 & 10,893 \\
\hline Total & 692,634 & 72,395 & 133,917 & 161,365 & 106,889 & 61,282 & 55,678 & 40,462 & 24,622 & 16,059 & 19,965 \\
\hline
\end{tabular}

${ }^{a}$ Classifications at the beginning of the remeasurement period. 
Table 20-Average annual removals of growing stock on timberland, by broad management class, species group, and stand-age class, Florida, 1987-1994

\begin{tabular}{|c|c|c|c|c|c|c|c|c|c|c|c|}
\hline \multirow{2}{*}{$\begin{array}{l}\text { Broad management } \\
\text { class }^{a} \text { and } \\
\text { species group }\end{array}$} & \multirow{2}{*}{$\begin{array}{c}\text { All } \\
\text { classes } \\
\end{array}$} & \multirow{2}{*}{$\begin{array}{c}\text { No } \\
\text { manageable } \\
\text { stand }\end{array}$} & \multicolumn{9}{|c|}{ Stand-age class (years) ${ }^{a}$} \\
\hline & & & $0-10$ & $11-20$ & $21-30$ & $31-40$ & $41-50$ & $51-60$ & $61-70$ & $71-80$ & $81+$ \\
\hline & \multicolumn{11}{|c|}{ Thousand cubic feet } \\
\hline \multicolumn{12}{|l|}{ Pine plantation } \\
\hline Softwood & 228,978 & 767 & 2,206 & 57,403 & 141,467 & 24,000 & 2,210 & 925 & - & - & - \\
\hline Hardwood & 2,162 & - & 44 & 280 & 1,624 & 158 & 56 & - & - & - & - \\
\hline Total & 231,140 & 767 & 2,250 & 57,683 & 143,091 & 24,158 & 2,266 & 925 & - & - & - \\
\hline \multicolumn{12}{|l|}{ Natural pine } \\
\hline Softwood & 168,192 & 18,255 & 2,820 & 5,125 & 22,005 & 51,290 & 40,488 & 17,209 & 9,188 & 1,161 & 651 \\
\hline Hardwood & 5,326 & 289 & - & 147 & 1,000 & 1,255 & 1,686 & 715 & 198 & 36 & - \\
\hline Total & 173,518 & 18,544 & 2,820 & 5,272 & 23,005 & 52,545 & 42,174 & 17,924 & 9,386 & 1,197 & 651 \\
\hline \multicolumn{12}{|l|}{ Oak-pine } \\
\hline Softwood & 26,588 & 5,726 & 937 & 1,184 & 2,828 & 3,093 & 7,875 & 3,866 & 956 & 123 & - \\
\hline Hardwood & 6,150 & 658 & 63 & 144 & 227 & 1,408 & 2,256 & 1,058 & 336 & - & - \\
\hline Total & 32,738 & 6,384 & 1,000 & 1,328 & 3,055 & 4,501 & 10,131 & 4,924 & 1,292 & 123 & - \\
\hline \multicolumn{12}{|l|}{ Upland hardwood } \\
\hline Softwood & 4,253 & 2,177 & 139 & 426 & 177 & 385 & 169 & 102 & 324 & - & 354 \\
\hline Hardwood & 16,618 & 4,846 & 700 & 1,605 & 436 & 2,467 & 2,429 & 1,665 & 351 & 74 & 2,045 \\
\hline Total & 20,871 & 7,023 & 839 & 2,031 & 613 & 2,852 & 2,598 & 1,767 & 675 & 74 & 2,399 \\
\hline \multicolumn{12}{|l|}{ Lowland hardwood } \\
\hline Softwood & 44,622 & 2,754 & - & 246 & 590 & 3,690 & 3,723 & 12,088 & 6,426 & 4,050 & 11,055 \\
\hline Hardwood & 57,830 & 5,575 & 375 & 506 & 702 & 5,385 & 8,187 & 17,170 & 10,513 & 3,694 & 5,723 \\
\hline Total & 102,452 & 8,329 & 375 & 752 & 1,292 & 9,075 & 11,910 & 29,258 & 16,939 & 7,744 & 16,778 \\
\hline \multicolumn{12}{|l|}{ All types } \\
\hline Softwood & 472,633 & 29,679 & 6,102 & 64,384 & 167,067 & 82,458 & 54,465 & 34,190 & 16,894 & 5,334 & 12,060 \\
\hline Hardwood & 88,086 & 11,368 & 1,182 & 2,682 & 3,989 & 10,673 & 14,614 & 20,608 & 11,398 & 3,804 & 7,768 \\
\hline Total & 560,719 & 41,047 & 7,284 & 67,066 & 171,056 & 93,131 & 69,079 & 54,798 & 28,292 & 9,138 & 19,828 \\
\hline
\end{tabular}


Table 21-Merchantable volume of live trees and growing stock on timberland, by forest-type and species groups, Florida, 1995

\begin{tabular}{|c|c|c|c|c|c|c|c|c|c|c|}
\hline \multirow[b]{2}{*}{ Forest-type group } & \multicolumn{5}{|c|}{ Live trees } & \multicolumn{5}{|c|}{ Growing stock } \\
\hline & $\begin{array}{c}\text { All } \\
\text { species }\end{array}$ & Pine & $\begin{array}{c}\text { Other } \\
\text { softwood }\end{array}$ & $\begin{array}{c}\text { Soft } \\
\text { hardwood }\end{array}$ & $\begin{array}{c}\text { Hard } \\
\text { hardwood }\end{array}$ & $\begin{array}{c}\text { All } \\
\text { species }\end{array}$ & Pine & $\begin{array}{c}\text { Other } \\
\text { softwood }\end{array}$ & $\begin{array}{c}\text { Soft } \\
\text { hardwood }\end{array}$ & $\begin{array}{c}\text { Hard } \\
\text { hardwood }\end{array}$ \\
\hline & \multicolumn{10}{|c|}{ Thousand cubic feet } \\
\hline Longleaf-slash pine & $4,922,176$ & $4,598,210$ & 57,307 & 127,829 & 138,830 & $4,823,488$ & $4,585,724$ & 53,468 & 116,633 & 67,663 \\
\hline Loblolly-shortleaf pine & $1,306,771$ & $1,190,948$ & 2,552 & 38,000 & 75,271 & $1,270,483$ & $1,183,853$ & 2,552 & 34,175 & 49,903 \\
\hline Oak-pine & $1,525,332$ & 759,545 & 116,889 & 301,345 & 347,553 & $1,347,450$ & 754,489 & 112,929 & 271,620 & 208,412 \\
\hline Oak-hickory & $1,745,055$ & 153,189 & 6,405 & 243,296 & $1,342,165$ & $1,257,449$ & 151,035 & 5,958 & 219,972 & 880,484 \\
\hline Oak-gum-cypress & $7,467,842$ & 299,509 & $2,337,649$ & $3,332,213$ & $1,498,471$ & $6,626,756$ & 298,332 & $2,272,652$ & $2,923,187$ & $1,132,585$ \\
\hline Elm-ash-cottonwood & 48,504 & 1,662 & 1,780 & 29,103 & 15,959 & 40,805 & 1,662 & 1,780 & 25,074 & 12,289 \\
\hline All types & $17,015,680$ & $7,003,063$ & $2,522,582$ & $4,071,786$ & $3,418,249$ & $15,366,431$ & $6,975,095$ & $2,449,339$ & $3,590,661$ & $2,351,336$ \\
\hline
\end{tabular}

Table 22-Area of timberland treated or disturbed annually and retained in timberland, by treatment or disturbance and ownership class, Florida, 1987 to 1995

\begin{tabular}{|c|c|c|c|c|c|}
\hline \multirow[b]{2}{*}{$\begin{array}{l}\text { Treatment or } \\
\text { disturbance }\end{array}$} & \multirow[b]{2}{*}{$\begin{array}{c}\text { All } \\
\text { ownerships }\end{array}$} & \multicolumn{4}{|c|}{ Ownership class } \\
\hline & & Public & $\begin{array}{c}\text { Forest } \\
\text { industry }\end{array}$ & $\begin{array}{c}\text { Forest } \\
\text { industry- } \\
\text { leased }\end{array}$ & $\begin{array}{l}\text { Other } \\
\text { private }\end{array}$ \\
\hline & \multicolumn{5}{|c|}{ Acres $^{B}$} \\
\hline Final harvest & 248,507 & 20,445 & 112,204 & 18,382 & 97,476 \\
\hline Partial harvest $^{b}$ & 21,495 & 2,621 & 5,448 & 1,728 & 11,698 \\
\hline Commercial thinning & 41,217 & 8,092 & 15,351 & 2,390 & 15,384 \\
\hline Other stand improvement & 6,254 & 956 & 1,603 & - & 3,695 \\
\hline Site preparation & 190,630 & 16,846 & 105,078 & 15,252 & 53,454 \\
\hline Artificial regeneration $^{c}$ & 239,762 & 16,861 & 113,772 & 13,448 & 95,681 \\
\hline Natural regeneration ${ }^{c}$ & 71,152 & 5,888 & 13,548 & 1,597 & 50,119 \\
\hline Other treatment & 73,832 & 12,415 & 12,796 & 1,514 & 47,107 \\
\hline Natural disturbance & 102,665 & 14,152 & 17,149 & 3,533 & 67,831 \\
\hline
\end{tabular}

Since some acres experience more than one treatment or disturbance, there are no column totals.

${ }^{b}$ Includes high-grading and some selective cutting.

${ }^{c}$ Includes establishment of trees for timber production on forest and nonforest land. 
Table 23-Area of timberland treated or disturbed annually and retained in timberland, by treatment or disturbance and broad management class, Florida, 1987 to 1995

\begin{tabular}{|c|c|c|c|c|c|c|}
\hline \multirow[b]{2}{*}{$\begin{array}{l}\text { Treatment or } \\
\text { disturbance }\end{array}$} & \multirow[b]{2}{*}{$\begin{array}{c}\text { All } \\
\text { classes }\end{array}$} & \multicolumn{5}{|c|}{ Broad management class ${ }^{a}$} \\
\hline & & $\begin{array}{c}\text { Pine } \\
\text { plantation }\end{array}$ & $\begin{array}{l}\text { Natural } \\
\text { pine }\end{array}$ & $\begin{array}{l}\text { Oak- } \\
\text { pine }\end{array}$ & $\begin{array}{c}\text { Upland } \\
\text { hardwood }\end{array}$ & $\begin{array}{l}\text { Lowland } \\
\text { hardwood }\end{array}$ \\
\hline & \multicolumn{6}{|c|}{ Acres $^{b}$} \\
\hline Final harvest & 248,507 & 116,176 & 80,239 & 12,947 & 5,943 & 33,202 \\
\hline Partial harvest ${ }^{c}$ & 21,495 & 381 & 7,867 & 4,798 & 1,670 & 6,779 \\
\hline Commercial thinning & 41,217 & 31,184 & 8,686 & 703 & - & 644 \\
\hline Other stand improvement & 6,254 & 2,510 & 1,794 & 729 & 809 & 412 \\
\hline Site preparation & 190,630 & 88,988 & 57,522 & 10,200 & 19,674 & 14,246 \\
\hline Other treatment & 73,832 & 1,916 & 29,677 & 11,933 & 13,988 & 16,318 \\
\hline Natural disturbance & 102,665 & 34,025 & 22,131 & 6,209 & 8,785 & 31,515 \\
\hline
\end{tabular}

${ }^{a}$ Classification before treatment or disturbance.

${ }^{b}$ Since some acres experience more than one treatment or disturbance, there are no column totals.

${ }^{c}$ Includes high-grading and some selective cutting.

Table 24-Area of timberland regenerated annually, by type of regeneration and broad management class, Florida, 1987 to 1995

\begin{tabular}{|c|c|c|c|c|c|c|}
\hline \multirow[b]{2}{*}{$\begin{array}{l}\text { Type of } \\
\text { regeneration }\end{array}$} & \multirow[b]{2}{*}{$\begin{array}{c}\text { All } \\
\text { classes }\end{array}$} & \multicolumn{5}{|c|}{ 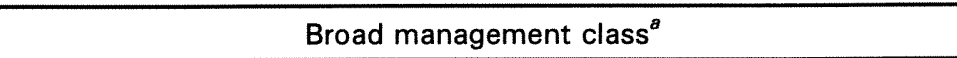 } \\
\hline & & $\begin{array}{c}\text { Pine } \\
\text { plantation }\end{array}$ & $\begin{array}{l}\text { Natural } \\
\text { pine }\end{array}$ & $\begin{array}{l}\text { Oak- } \\
\text { pine }\end{array}$ & $\begin{array}{c}\text { Upland } \\
\text { hardwood }\end{array}$ & $\begin{array}{l}\text { Lowland } \\
\text { hardwood }\end{array}$ \\
\hline & \multicolumn{6}{|c|}{ Acres } \\
\hline $\begin{array}{c}\text { Artificial regeneratio } \\
\text { following harvest }\end{array}$ & 123,234 & 112,314 & - & 8,355 & 1,976 & 589 \\
\hline $\begin{array}{c}\text { Natural regeneration } \\
\text { following harvest }\end{array}$ & 30,302 & - & 7,259 & 3,442 & 11,627 & 7,974 \\
\hline $\begin{array}{l}\text { Other artificial regen } \\
\text { on forest land }\end{array}$ & 74,510 & 67,592 & - & 6,918 & - & - \\
\hline $\begin{array}{l}\text { Other natural regene } \\
\text { on forest land }\end{array}$ & 27,891 & - & 12,447 & 4,848 & 6,212 & 4,384 \\
\hline $\begin{array}{c}\text { Artificial regeneratio } \\
\text { nonforest land }\end{array}$ & 42,018 & 40,237 & - & 1,781 & - & - \\
\hline $\begin{array}{c}\text { Natural reversion of } \\
\text { nonforest land }\end{array}$ & 12,959 & - & 7,252 & 2,205 & 2,040 & 1,462 \\
\hline Total & 310,914 & 220,143 & 26,958 & 27,549 & 21,855 & 14,409 \\
\hline
\end{tabular}

${ }^{a}$ Classification after regeneration. 
Table 25-Area of timberland, by treatment opportunity and broad management classes, Florida, 1995

\begin{tabular}{|c|c|c|c|c|c|c|}
\hline \multirow[b]{2}{*}{$\begin{array}{l}\text { Treatment } \\
\text { opportunity class }\end{array}$} & \multirow[b]{2}{*}{$\begin{array}{c}\text { All } \\
\text { classes }\end{array}$} & \multicolumn{5}{|c|}{ Broad management class } \\
\hline & & $\begin{array}{c}\text { Pine } \\
\text { plantation }\end{array}$ & $\begin{array}{l}\text { Natural } \\
\text { pine }\end{array}$ & $\begin{array}{l}\text { Oak- } \\
\text { pine }\end{array}$ & $\begin{array}{c}\text { Upland } \\
\text { hardwood }\end{array}$ & $\begin{array}{l}\text { Lowland } \\
\text { hardwood }\end{array}$ \\
\hline & \multicolumn{6}{|c|}{ Acres } \\
\hline Salvage & 65,486 & 15,818 & 20,664 & 2,362 & 2,472 & 24,170 \\
\hline Harvest & 574,915 & 2,579 & 78,824 & 65,807 & 70,016 & 357,689 \\
\hline Commercial thinning & 550,561 & 460,179 & 53,651 & 2,916 & - & 33,815 \\
\hline Other stand improvement & 730,511 & 155,296 & 174,700 & 97,904 & 159,244 & 143,367 \\
\hline Stand conversion & 79,973 & 12,767 & - & 22,095 & 27,323 & 17,788 \\
\hline Regeneration & $3,733,346$ & 72,066 & 868,941 & 606,698 & $1,249,129$ & 936,512 \\
\hline \multicolumn{7}{|l|}{ Stand in relatively } \\
\hline good condition & $7,988,630$ & $3,915,022$ & $1,584,593$ & 660,109 & 505,159 & $1,323,747$ \\
\hline Adverse sites $^{a}$ & 927,238 & - & 22,688 & 21,089 & - & 883,461 \\
\hline All classes & $14,650,660$ & $4,633,727$ & $2,804,061$ & $1,478,980$ & $2,013,343$ & $3,720,549$ \\
\hline
\end{tabular}

a Areas where management opportunities are severely limited because of steep slopes or poor drainage.

Table 26-Area of timberland, by treatment opportunity and ownership classes, Florida, 1995

\begin{tabular}{|c|c|c|c|c|c|}
\hline \multirow[b]{2}{*}{$\begin{array}{l}\text { Treatment } \\
\text { opportunity class }\end{array}$} & \multirow[b]{2}{*}{$\begin{array}{c}\text { All } \\
\text { ownerships }\end{array}$} & \multicolumn{4}{|c|}{ Ownership class } \\
\hline & & Public & $\begin{array}{l}\text { Forest } \\
\text { industry }\end{array}$ & $\begin{array}{c}\text { Forest } \\
\text { industry- } \\
\text { leased }\end{array}$ & $\begin{array}{l}\text { Other } \\
\text { private }\end{array}$ \\
\hline & \multicolumn{5}{|c|}{ Acres } \\
\hline Salvage & 65,486 & 13,064 & 23,748 & - & 28,674 \\
\hline Harvest & 574,915 & 171,278 & 101,008 & 2,847 & 299,782 \\
\hline Commercial thinning & 550,561 & 49,399 & 220,819 & 56,736 & 223,607 \\
\hline Other stand improvement & 730,511 & 136,729 & 149,044 & 26,727 & 418,011 \\
\hline Stand conversion & 79,973 & 11,402 & 24,200 & - & 44,371 \\
\hline Regeneration & $3,733,346$ & 777,946 & 667,120 & 57,409 & $2,230,871$ \\
\hline \multicolumn{6}{|l|}{ Stand in relatively } \\
\hline good condition & $7,988,630$ & $1,398,256$ & $2,607,017$ & 412,175 & $3,571,182$ \\
\hline Adverse sites $^{a}$ & 927,238 & 273,698 & 222,550 & 30,083 & 400,907 \\
\hline All classes & $14,650,660$ & $2,831,772$ & $4,015,506$ & 585,977 & $7,217,405$ \\
\hline
\end{tabular}

a Areas where management opportunities are severely limited because of steep slopes or poor drainage. 
Table 27-Merchantable volume of live trees and growing stock on timberland, by ownership class and species group, Florida, 1995

\begin{tabular}{|c|c|c|c|c|c|c|c|c|c|c|}
\hline \multirow[b]{2}{*}{ Ownership class } & \multicolumn{5}{|c|}{ Live trees } & \multicolumn{5}{|c|}{ Growing stock } \\
\hline & $\begin{array}{c}\text { All } \\
\text { species }\end{array}$ & Pine & $\begin{array}{c}\text { Other } \\
\text { softwood }\end{array}$ & $\begin{array}{c}\text { Soft } \\
\text { hardwood }\end{array}$ & $\begin{array}{c}\text { Hard } \\
\text { hardwood }\end{array}$ & $\begin{array}{c}\text { All } \\
\text { species }\end{array}$ & Pine & $\begin{array}{c}\text { Other } \\
\text { softwood }\end{array}$ & $\begin{array}{c}\text { Soft } \\
\text { hardwood }\end{array}$ & $\begin{array}{c}\text { Hard } \\
\text { hardwood }\end{array}$ \\
\hline & \multicolumn{10}{|c|}{ Thousand cubic feet } \\
\hline National forest & $1,338,104$ & 865,532 & 140,866 & 251,145 & 80,561 & $1,263,662$ & 864,070 & 130,912 & 216,194 & 52,486 \\
\hline Other public & $2,921,530$ & $1,096,983$ & 465,631 & 753,717 & 605,199 & $2,607,402$ & $1,092,836$ & 449,624 & 664,242 & 400,700 \\
\hline Forest industry & $3,776,745$ & $1,847,574$ & 484,150 & 955,262 & 489,759 & $3,541,516$ & $1,840,314$ & 471,036 & 859,168 & 370,998 \\
\hline Forest industry-leased & 500,421 & 253,078 & 56,644 & 127,955 & 62,744 & 476,442 & 252,444 & 56,181 & 117,363 & 50,454 \\
\hline Other private & $8,478,880$ & $2,939,896$ & $1,375,291$ & $1,983,707$ & $2,179,986$ & $7,477,409$ & $2,925,431$ & $1,341,586$ & $1,733,694$ & $1,476,698$ \\
\hline All ownerships & $17,015,680$ & $7,003,063$ & $2,522,582$ & $4,071,786$ & $3,418,249$ & $15,366,431$ & $6,975,095$ & $2,449,339$ & $3,590,661$ & $2,351,336$ \\
\hline
\end{tabular}

Table 28-Volume of sawtimber on timberland, by ownership class and species group, Fiorida, 1995

\begin{tabular}{|c|c|c|c|c|c|c|c|c|c|c|}
\hline \multirow[b]{2}{*}{ Ownership class } & \multicolumn{5}{|c|}{ Small sawtimber } & \multicolumn{5}{|c|}{ Large sawtimber ${ }^{b}$} \\
\hline & $\begin{array}{c}\text { All } \\
\text { species }\end{array}$ & Pine & $\begin{array}{c}\text { Other } \\
\text { sof twood }\end{array}$ & $\begin{array}{c}\text { Soft } \\
\text { hardwood }\end{array}$ & $\begin{array}{c}\text { Hard } \\
\text { hardwood }\end{array}$ & $\begin{array}{c}\text { All } \\
\text { species }\end{array}$ & Pine & $\begin{array}{c}\text { Other } \\
\text { softwood }\end{array}$ & $\begin{array}{c}\text { Soft } \\
\text { hardwood }\end{array}$ & $\begin{array}{c}\text { Hard } \\
\text { hardwood }\end{array}$ \\
\hline & \multicolumn{10}{|c|}{ Thousand board feet } \\
\hline National forest & $2,609,666$ & $2,116,546$ & 235,603 & 200,767 & 56.750 & $1,417,278$ & 837,605 & 171,938 & 291,467 & 116,268 \\
\hline Other public & $4,579,364$ & $2,591,402$ & 860,869 & 767,707 & 359,386 & $4,393,318$ & $1,675,093$ & 665,170 & $1,097,370$ & 955,685 \\
\hline Forest industry & $5,081,181$ & $2,875,505$ & 892,278 & 959,441 & 353,957 & $3,603,869$ & $1,052,572$ & 513,402 & $1,135,443$ & 902,452 \\
\hline Forest industry-leased & 560,693 & 282,515 & 111,729 & 120,873 & 45,576 & 453,913 & 196,753 & 35,332 & 93,755 & 128,073 \\
\hline Other private & $11,295,986$ & $5,387,924$ & $2,576,041$ & $1,925,474$ & $1,406,547$ & $11,291,962$ & $3,715,232$ & $1,518,030$ & $2,411,537$ & $3,647,163$ \\
\hline All ownerships & $24,126,890$ & $13,253,892$ & $4,676,520$ & $3,974,262$ & $2,222,216$ & $21,160,340$ & $7,477,255$ & $2,903,872$ & $5,029,572$ & $5,749,641$ \\
\hline
\end{tabular}

- Volume of sawtimber trees less than 15.0 inches at d.b.h.

${ }^{b}$ Volume of sawtimber trees 15.0 inches and larger at d.b.h.

Table 29-Average net annual growth and removals of growing stock on timberland, by ownership class and species group, Florida, 1987-1994

\begin{tabular}{|c|c|c|c|c|c|c|c|c|c|c|}
\hline \multirow[b]{2}{*}{ Ownership class } & \multicolumn{5}{|c|}{ Net annual growth } & \multicolumn{5}{|c|}{ Annual timber removals } \\
\hline & $\begin{array}{c}\text { All } \\
\text { species }\end{array}$ & Pine & $\begin{array}{c}\text { Other } \\
\text { softwood }\end{array}$ & $\begin{array}{c}\text { Soft } \\
\text { hardwood }\end{array}$ & $\begin{array}{c}\text { Hard } \\
\text { hardwood }\end{array}$ & $\begin{array}{c}\text { All } \\
\text { species }\end{array}$ & Pine & $\begin{array}{l}\text { Other } \\
\text { softwood }\end{array}$ & $\begin{array}{c}\text { Soft } \\
\text { hardwood }\end{array}$ & $\begin{array}{c}\text { Hard } \\
\text { hardwood }\end{array}$ \\
\hline & \multicolumn{10}{|c|}{ Thousand cubic feet } \\
\hline National forest & 32,823 & 26,373 & 1,370 & 3,894 & 1,186 & 20,500 & 20,500 & - & - & - \\
\hline Other public & 69,732 & 39,454 & 7,519 & 12,490 & 10,269 & 47,056 & 30,842 & 3,439 & 4,865 & 7,910 \\
\hline Forest industry & 229,775 & 188,369 & 6,685 & 20,575 & 14,146 & 207,973 & 172,210 & 11,054 & 12,024 & 12,685 \\
\hline Forest industry-leased & 36,554 & 30,153 & 755 & 4,012 & 1,634 & 36,597 & 31,189 & 2,571 & 2,503 & 334 \\
\hline Other private & 323,750 & 204,375 & 26,700 & 42,114 & 50,561 & 248,593 & 176,695 & 24,133 & 21,446 & 26,319 \\
\hline All ownerships & 692,634 & 488,724 & 43,029 & 83,085 & 77,796 & 560,719 & 431,436 & 41,197 & 40,838 & 47,248 \\
\hline
\end{tabular}


Table 30-Average net annual growth and removals of sawtimber on timberland, by ownership class and species group, Florida, $1987-1994$

\begin{tabular}{|c|c|c|c|c|c|c|c|c|c|c|}
\hline \multirow[b]{2}{*}{ Ownership class } & \multicolumn{5}{|c|}{ Net annual growth } & \multicolumn{5}{|c|}{ Annual timber removals } \\
\hline & $\begin{array}{c}\text { All } \\
\text { species }\end{array}$ & Pine & $\begin{array}{c}\text { Other } \\
\text { softwood }\end{array}$ & $\begin{array}{c}\text { Soft } \\
\text { hardwood }\end{array}$ & $\begin{array}{c}\text { Hard } \\
\text { hardwood }\end{array}$ & $\begin{array}{c}\text { All } \\
\text { species }\end{array}$ & Pine & $\begin{array}{c}\text { Other } \\
\text { softwood }\end{array}$ & $\begin{array}{c}\text { Soft } \\
\text { hardwood }\end{array}$ & $\begin{array}{c}\text { Hard } \\
\text { hardwood }\end{array}$ \\
\hline & \multicolumn{10}{|c|}{ Thousand board feet } \\
\hline National forest & 104,010 & 82,805 & 5,653 & 10,229 & 5,323 & 56,716 & 56,716 & - & - & - \\
\hline Other public & 268,748 & 144,524 & 37,244 & 45,436 & 41,544 & 162,643 & 107.564 & 10,598 & 15,336 & 29,145 \\
\hline Forest industry & 527,853 & 389,906 & 33,402 & 55,857 & 48,688 & 450,970 & 349,053 & 34,588 & 32,362 & 34,967 \\
\hline Forest industry-leased & 75,520 & 55,713 & 3,476 & 11,071 & 5,260 & 59,494 & 48,587 & 5,561 & 4,541 & 805 \\
\hline Other private & 989,047 & 552,779 & 116,709 & 127,333 & 192,226 & 753,111 & 541,634 & 63,594 & 58,916 & 88,967 \\
\hline All ownerships & $1,965,178$ & $1,225,727$ & 196,484 & 249,926 & 293,041 & $1,482,934$ & $1,103,554$ & 114,341 & 111,155 & 153,884 \\
\hline
\end{tabular}

Table 31-Volume of timber on timberland, by class of timber and species group, Florida, 1995

\begin{tabular}{|c|c|c|c|c|c|}
\hline Class of timber & $\begin{array}{c}\text { All } \\
\text { species }\end{array}$ & Pine & $\begin{array}{c}\text { Other } \\
\text { softwood }\end{array}$ & $\begin{array}{c}\text { Soft } \\
\text { hardwood }\end{array}$ & $\begin{array}{c}\text { Hard } \\
\text { hardwood }\end{array}$ \\
\hline
\end{tabular}

Sawtimber trees

Saw-log portion

Upper-stem portion ${ }^{a}$

Total

\begin{tabular}{rrrrr}
$8,584,865$ & $3,810,749$ & $1,563,513$ & $1,762,254$ & $1,448,349$ \\
$1,201,485$ & 390,683 & 220,361 & 363,198 & 227,243 \\
\hline $9,786,350$ & $4,201,432$ & $1,783,874$ & $2,125,452$ & $1,675,592$ \\
$5,580,081$ & $2,773,663$ & 665,465 & $1,465,209$ & 675,744 \\
\hline $15,366,431$ & $6,975,095$ & $2,449,339$ & $3,590,661$ & $2,351,336$ \\
\hline \hline
\end{tabular}

Rough trees

Sawtimber size

Poletimber size

Total

\begin{tabular}{rllll}
759,290 & 11,710 & 21,478 & 170,389 & 555,713 \\
695,916 & 14,603 & 22,667 & 230,128 & 428,518 \\
\hline $1,455,206$ & 26,313 & 44,145 & 400,517 & 984,231 \\
\hline \hline
\end{tabular}

\section{Rotten trees}

Sawtimber size

Poletimber size

Total

\begin{tabular}{rrrrr}
171,138 & 1,212 & 26,072 & 68,349 & 75,505 \\
22,905 & 443 & 3,026 & 12,259 & 7,177 \\
\hline 194,043 & 1,655 & 29,098 & 80,608 & 82,682 \\
\hline \hline
\end{tabular}

\section{Salvable dead trees}

Sawtimber size
Poletimber size

Total

Total, all timber

\begin{tabular}{rrrrr}
7,024 & 4,751 & 649 & 977 & 647 \\
3,958 & 2,354 & 732 & 565 & 307 \\
\hline 10,982 & 7,105 & 1,381 & 1,542 & 954 \\
\hline \hline & & & & \\
$17,026,662$ & $7,010,168$ & $2,523,963$ & $4,073,328$ & $3,419,203$ \\
\hline
\end{tabular}

\footnotetext{
${ }^{a}$ Includes cull sections in the saw-log portion.
} 
Table 32-Number of live trees on timberland, by species and diameter class, Florida, 1995

\begin{tabular}{|c|c|c|c|c|c|c|c|c|c|c|c|c|c|}
\hline \multirow[b]{2}{*}{ Species } & \multirow[b]{2}{*}{$\begin{array}{c}\text { All } \\
\text { classes }\end{array}$} & \multicolumn{12}{|c|}{ Diameter class (inches at breast height) } \\
\hline & & $\begin{array}{l}1.0- \\
2.9 \\
\end{array}$ & $\begin{array}{l}3.0- \\
4.9 \\
\end{array}$ & $\begin{array}{l}5.0- \\
6.9 \\
\end{array}$ & $\begin{array}{l}7.0- \\
8.9 \\
\end{array}$ & $\begin{array}{r}9.0- \\
10.9 \\
\end{array}$ & $\begin{array}{l}11.0- \\
12.9 \\
\end{array}$ & $\begin{array}{l}13.0- \\
14.9\end{array}$ & $\begin{array}{l}15.0- \\
16.9 \\
\end{array}$ & $\begin{array}{l}17.0- \\
18.9 \\
\end{array}$ & $\begin{array}{l}19.0- \\
20.9 \\
\end{array}$ & $\begin{array}{l}21.0- \\
28.9 \\
\end{array}$ & $\begin{array}{c}29.0 \text { and } \\
\text { larger }\end{array}$ \\
\hline & \multicolumn{13}{|c|}{ Thousand trees } \\
\hline \multicolumn{14}{|l|}{ Softwood } \\
\hline Longleaf pine & 179,618 & 75,181 & 34,336 & 17,424 & 11,961 & 13,737 & 13,139 & 8,624 & 3,764 & 993 & 346 & 113 & - \\
\hline Slash pine & $1,752,081$ & 530,791 & 547,152 & 381,953 & 177,263 & 63,338 & 26,989 & 13,280 & 6,453 & 2,997 & 1,188 & 666 & 11 \\
\hline Shortleaf pine & 4,483 & 1,846 & 499 & 624 & 506 & 155 & 234 & 238 & 216 & 76 & 49 & 40 & - \\
\hline Loblolly pine & 327,082 & 118,507 & 107,765 & 57,262 & 20,255 & 9,103 & 5,502 & 3,261 & 2,301 & 1,284 & 915 & 897 & 30 \\
\hline Pond pine & 24,930 & 5,012 & 6,423 & 3,780 & 3,701 & 2,334 & 1,549 & 1,188 & 517 & 255 & 85 & 86 & - \\
\hline Virginia pine & - & - & - & - & - & - & - & - & - & - & - & - & - \\
\hline Pitch pine & - & - & - & - & - & - & - & - & - & - & - & - & - \\
\hline Table Mountain pine & - & - & - & - & - & - & - & - & - & - & - & - & - \\
\hline Spruce pine & 5,357 & 3,310 & 659 & 211 & 521 & 149 & 133 & 111 & 79 & 82 & 57 & 41 & 4 \\
\hline Sand pine & 342,365 & 169,185 & 101,460 & 42,125 & 18,010 & 6,026 & 3,095 & 1,410 & 712 & 214 & 107 & 21 & - \\
\hline Eastern white pine & - & - & - & - & - & - & - & - & - & - & - & - & - \\
\hline Eastern hemlock & - & - & - & - & - & - & - & - & - & - & - & - & - \\
\hline Spruce and fir & - & - & - & - & - & - & - & - & - & - & - & - & - \\
\hline Baldcypress & 68,636 & 18,232 & 14,169 & 9,606 & 8,145 & 6,177 & 4,391 & 3,567 & 1,966 & 1,089 & 595 & 594 & 105 \\
\hline Pondcypress & 591,798 & 264,064 & 136,498 & 74,663 & 48,761 & 31,565 & 18,517 & 9,962 & 4,261 & 1,784 & 885 & 776 & 62 \\
\hline Cedars & 33,503 & 16,750 & 6,153 & 3,217 & 2,331 & 1,998 & 1,375 & 852 & 401 & 211 & 132 & 79 & 4 \\
\hline Total softwoods & $3,329,853$ & $1,202,878$ & 955,114 & 590,865 & 291,454 & 134,582 & 74,924 & 42,493 & 20,670 & 8,985 & 4,359 & 3,313 & 216 \\
\hline \multicolumn{14}{|l|}{ Hardwood } \\
\hline Select white oaks & 8,555 & 3,955 & 1,982 & 1,183 & 539 & 235 & 268 & 121 & 89 & 74 & 58 & 44 & 7 \\
\hline Select red oaks & 430 & 340 & - & - & 67 & - & - & 16 & - & - & - & 7 & - \\
\hline Chestnut oak & - & - & - & - & - & - & - & - & - & - & - & - & - \\
\hline Other white oaks & 229,683 & 123,330 & 50,582 & 20,579 & 9,833 & 6,476 & 4,555 & 3,216 & 3,134 & 2,064 & 1,608 & 3,176 & 1,130 \\
\hline Other red oaks & 830,230 & 574,266 & 126,593 & 51,582 & 26,785 & 17,905 & 13,001 & 7,724 & 4,677 & 3,091 & 1,892 & 2,274 & 440 \\
\hline Hickory & 30,755 & 18,307 & 3,806 & 3,340 & 1,359 & 1,312 & 639 & 774 & 480 & 330 & 164 & 233 & 11 \\
\hline Yellow birch & - & - & - & - & - & - & - & - & - & - & - & - & - \\
\hline Hard maple & 6,184 & 4,056 & 848 & 485 & 307 & 124 & 112 & 146 & 20 & 39 & 9 & 38 & - \\
\hline Soft maple & 283,167 & 177,912 & 52,083 & 20,518 & 12,724 & 8,202 & 4,245 & 3,496 & 1,928 & 932 & 575 & 507 & 45 \\
\hline Beech & 1,993 & 1,317 & 331 & 83 & 63 & 39 & - & 37 & 28 & 24 & 17 & 50 & 4 \\
\hline Sweetgum & 233,007 & 138,247 & 45,382 & 19,950 & 9,983 & 7,889 & 4,744 & 3,371 & 1,704 & 796 & 471 & 442 & 28 \\
\hline Tupelo and blackgum & 716,527 & 379,052 & 164,292 & 74,373 & 40,938 & 22,740 & 15,450 & 9,305 & 4,997 & 2,666 & 1,272 & 1,293 & 149 \\
\hline Ash & 252,925 & 148,755 & 55,672 & 23,745 & 10,345 & 6,538 & 3,514 & 1,798 & 1,208 & 671 & 347 & 313 & 19 \\
\hline Cottonwood & 419 & 342 & - & - & 71 & - & - & - & - & - & - & 6 & - \\
\hline Basswood & 5,381 & 2,911 & 1,303 & 328 & 354 & 107 & 88 & 118 & 92 & 47 & 10 & 23 & - \\
\hline Yellow-poplar & 15,372 & 6,759 & 3,336 & 1,842 & 787 & 1,068 & 528 & 385 & 233 & 184 & 134 & 108 & 8 \\
\hline Bay and magnolia & 576,636 & 338,705 & 123,621 & 53,658 & 25,872 & 14,969 & 9,282 & 5,278 & 2,626 & 1,299 & 628 & 656 & 42 \\
\hline Black cherry & 28,693 & 19,341 & 5,667 & 1,890 & 1,076 & 409 & 130 & 76 & 57 & 36 & 11 & - & - \\
\hline Black walnut & 441 & 168 & 168 & 105 & - & - & - & - & - & - & - & - & - \\
\hline Sycamore & 411 & - & 157 & - & 72 & - & 47 & 39 & 15 & 35 & 19 & 19 & 8 \\
\hline Black locust & - & - & - & - & - & - & - & - & - & - & - & - & - \\
\hline Elm & 43,750 & 21,310 & 12,040 & 4,389 & 2,336 & 1,516 & 768 & 629 & 333 & 211 & 89 & 121 & 8 \\
\hline Other Eastern hardwoods & $1,039,628$ & 778,790 & 175,357 & 52,195 & 18,156 & 8,732 & 3,095 & 1,641 & 769 & 428 & 254 & 176 & 35 \\
\hline Total hardwoods & $4,304,187$ & $2,737,863$ & 823,220 & 330,245 & 161,667 & 98,261 & 60,466 & 38,170 & 22,390 & 12,927 & 7,558 & 9,486 & 1,934 \\
\hline All species & $7,634,040$ & $3,940,741$ & $1,778,334$ & 921,110 & 453,121 & 232,843 & 135,390 & 80,663 & 43,060 & 21,912 & 11,917 & 12,799 & 2,150 \\
\hline
\end{tabular}


Table 33-Number of growing-stock trees on timberland, by species and diameter class, Florida, 1995

\begin{tabular}{|c|c|c|c|c|c|c|c|c|c|c|c|c|c|}
\hline \multirow[b]{2}{*}{ Species } & \multirow[b]{2}{*}{$\begin{array}{c}\text { All } \\
\text { classes }\end{array}$} & \multicolumn{12}{|c|}{ Diameter class (inches at breast height) } \\
\hline & & $\begin{array}{l}1.0- \\
2.9\end{array}$ & $\begin{array}{l}3.0- \\
4.9\end{array}$ & $\begin{array}{l}5.0- \\
6.9 \\
\end{array}$ & $\begin{array}{l}7.0- \\
8.9\end{array}$ & $\begin{array}{r}9.0- \\
10.9\end{array}$ & $\begin{array}{l}11.0- \\
12.9\end{array}$ & $\begin{array}{l}13.0- \\
14.9\end{array}$ & $\begin{array}{l}15.0- \\
16.9\end{array}$ & $\begin{array}{l}17.0- \\
18.9\end{array}$ & $\begin{array}{l}19.0- \\
20.9\end{array}$ & $\begin{array}{l}21.0- \\
28.9\end{array}$ & $\begin{array}{c}29.0 \text { and } \\
\text { larger }\end{array}$ \\
\hline & \multicolumn{13}{|c|}{ Thousand trees } \\
\hline \multicolumn{14}{|l|}{ Softwood } \\
\hline Longleaf pine & 175,735 & 71,885 & 34,171 & 17,120 & 11,961 & 13,737 & 13,062 & 8,605 & 3,747 & 993 & 346 & 108 & - \\
\hline Slash pine & $1,726,560$ & 513,651 & 542,401 & 379,127 & 176,824 & 63,068 & 26,966 & 13,241 & 6,438 & 2,985 & 1,188 & 660 & 11 \\
\hline Shortleaf pine & 4,068 & 1,508 & 499 & 624 & 429 & 155 & 234 & 238 & 216 & 76 & 49 & 40 & - \\
\hline Loblolly pine & 320,793 & 114,915 & 106,302 & 56,344 & 20,111 & 8,986 & 5,472 & 3,242 & 2,301 & 1,284 & 915 & 891 & 30 \\
\hline Pond pine & 22,575 & 4,070 & 5,479 & 3,408 & 3,650 & 2,301 & 1,549 & 1,188 & 504 & 255 & 85 & 86 & - \\
\hline Virginia pine & - & - & - & - & - & - & - & - & - & - & - & - & - \\
\hline Pitch pine & - & - & - & - & - & - & - & - & - & - & - & - & - \\
\hline Table Mountain pine & - & - & - & - & - & - & - & - & - & - & - & - & - \\
\hline Spruce pine & 4,528 & 2,481 & 659 & 211 & 521 & 149 & 133 & 111 & 79 & 82 & 57 & 41 & 4 \\
\hline Sand pine & 328,971 & 158,094 & 100,041 & 41,702 & 17,745 & 5,914 & 3,028 & 1,393 & 712 & 214 & 107 & 21 & - \\
\hline Eastern white pine & - & - & - & - & - & - & 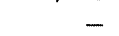 & - & - & - & - & - & - \\
\hline Eastern hemlock & - & - & - & - & - & - & - & - & - & - & - & - & - \\
\hline Spruce and fir & - & - & - & - & - & - & - & - & - & - & - & - & - \\
\hline Baldcypress & 61,384 & 12,910 & 12,851 & 9,467 & 7,945 & 6,177 & 4,368 & 3,505 & 1,919 & 1,078 & 595 & 533 & 36 \\
\hline Pondcypress & 531,362 & 225,867 & 124,456 & 69,650 & 46,319 & 30,304 & 17,977 & 9,642 & 3,975 & 1,652 & 817 & 663 & 40 \\
\hline Cedars & 29,991 & 15,378 & 4,875 & 2,991 & 1,975 & 1,887 & 1,222 & 852 & 385 & 211 & 132 & 79 & 4 \\
\hline Total softwoods & $3,205,967$ & $1,120,759$ & 931.734 & 580,644 & 287,480 & 132,678 & 74,011 & 42,017 & 20,276 & 8,830 & 4,291 & 3,122 & 125 \\
\hline \multicolumn{14}{|l|}{ Hardwood } \\
\hline Select white oaks & 5,935 & 2,137 & 1,477 & 986 & 472 & 235 & 241 & 121 & 89 & 74 & 58 & 38 & 7 \\
\hline Select red oaks & 90 & - & - & - & 67 & - & - & 16 & - & - & - & 7 & _- \\
\hline Chestnut oak & - & - & - & - & - & - & - & - & - & - & - & - & - \\
\hline Other white oaks & 42,018 & 16,075 & 9,070 & 4,269 & 3,239 & 1,799 & 1,165 & 1,254 & 1,320 & 885 & 737 & 1,617 & 588 \\
\hline Other red oaks & 531,889 & 327,027 & 96,606 & 42,201 & 22,391 & 15,816 & 11,352 & 6,597 & 3,835 & 2,519 & 1,535 & 1,732 & 278 \\
\hline Hickory & 14,138 & 4,974 & 1,746 & 2,802 & 995 & 1,204 & 586 & 719 & 408 & 318 & 155 & 220 & 11 \\
\hline Yellow birch & - & - & - & - & - & - & - & - & - & - & - & - & - \\
\hline Hard maple & 1,238 & 321 & - & 274 & 307 & 87 & 57 & 86 & 20 & 39 & 9 & 38 & - \\
\hline Soft maple & 121,155 & 56,079 & 28,365 & 12,732 & 8,953 & 6,048 & 3,237 & 2,695 & 1,392 & 766 & 464 & 399 & 25 \\
\hline Beech & 1,121 & 652 & 175 & 83 & 63 & 39 & - & 37 & - & 24 & 17 & 27 & 4 \\
\hline Sweetgum & 167,608 & 85,861 & 34,994 & 18,644 & 9,496 & 7,541 & 4,613 & 3,216 & 1,646 & 735 & 462 & 385 & 15 \\
\hline Tupelo and blackgum & 391,743 & 132,619 & 112,889 & 61,084 & 34,935 & 19,995 & 13,317 & 8,241 & 4,402 & 2,259 & 998 & 918 & 86 \\
\hline Ash & 86,315 & 37,784 & 18,589 & 12,121 & 6,393 & 5,051 & 2,932 & 1,403 & 940 & 602 & 256 & 241 & 3 \\
\hline Cottonwood & 71 & - & - & - & 71 & - & - & - & - & - & - & - & - \\
\hline Basswood & 2,920 & 1,616 & 644 & 125 & 177 & 65 & 50 & 98 & 77 & 35 & 10 & 23 & - \\
\hline Yellow-poplar & 13,806 & 5,768 & 2,851 & 1,842 & 731 & 1,068 & 528 & 385 & 233 & 184 & 125 & 83 & 8 \\
\hline Bay and magnolia & 368,085 & 193,993 & 80,789 & 42,608 & 21,480 & 12,196 & 8,072 & 4,542 & 2,200 & 1,172 & 470 & 529 & 34 \\
\hline Black cherry & 15,437 & 8,189 & 4,058 & 1,543 & 1,012 & 361 & 105 & 76 & 57 & 25 & 11 & - & - \\
\hline Black walnut & - & - & - & - & - & - & - & - & - & - & - & - & - \\
\hline Sycamore & 411 & - & 157 & - & 72 & - & 47 & 39 & 15 & 35 & 19 & 19 & 8 \\
\hline Black locust & - & - & - & - & - & - & - & - & - & - & - & - & - \\
\hline Elm & 21,169 & 7,137 & 6,736 & 2,645 & 1,855 & 1,116 & 472 & 588 & 275 & 165 & 71 & 105 & 4 \\
\hline Other Eastern hardwoods & 19,779 & 9,191 & 5,186 & 2,447 & 1,200 & 672 & 472 & 334 & 167 & 35 & 45 & 30 & - \\
\hline Total hardwoods & $1,804,928$ & 889,423 & 404,332 & 206,406 & 113,909 & 73,293 & 47,246 & 30,447 & 17,076 & 9,872 & 5,442 & 6,411 & 1,071 \\
\hline All species & $5,010,895$ & $2,010,182$ & $1,336,066$ & 787,050 & 401,389 & 205,971 & 121,257 & 72,464 & 37,352 & 18,702 & 9,733 & 9,533 & 1,196 \\
\hline
\end{tabular}


Table 34-Merchantable volume of live trees on timberland, by species and diameter class, Florida, 1995

\begin{tabular}{|c|c|c|c|c|c|c|c|c|c|c|c|}
\hline \multirow[b]{2}{*}{ Species } & \multirow[b]{2}{*}{$\begin{array}{c}\text { All } \\
\text { classes }\end{array}$} & \multicolumn{10}{|c|}{ Diameter class (inches at breast height) } \\
\hline & & $\begin{array}{l}5.0- \\
6.9 \\
\end{array}$ & $\begin{array}{l}7.0- \\
8.9 \\
\end{array}$ & $\begin{array}{c}9.0- \\
10.9 \\
\end{array}$ & $\begin{array}{l}11.0- \\
12.9 \\
\end{array}$ & $\begin{array}{l}13.0- \\
14.9 \\
\end{array}$ & $\begin{array}{l}15.0 \\
16.9 \\
\end{array}$ & $\begin{array}{l}17.0- \\
18.9 \\
\end{array}$ & $\begin{array}{l}19.0- \\
20.9 \\
\end{array}$ & $\begin{array}{l}21.0- \\
28.9 \\
\end{array}$ & $\begin{array}{l}29.0 \text { and } \\
\text { larger }\end{array}$ \\
\hline & \multicolumn{11}{|c|}{ Thousand cubic feet } \\
\hline \multicolumn{12}{|l|}{ Softwood } \\
\hline Longleaf pine & $1,060,209$ & 49,323 & 83,528 & 183,216 & 268,901 & 251,461 & 145,298 & 49,284 & 20,112 & 9,086 & - \\
\hline Slash pine & $4,317,503$ & 968,493 & $1,114,448$ & 758,492 & 529,557 & 387,409 & 259,257 & 156,378 & 78,675 & 62,704 & 2,090 \\
\hline Shortleaf pine & 39,444 & 1,734 & 3,026 & 1,884 & 5,010 & 7,882 & 9,133 & 3,907 & 3,262 & 3,606 & - \\
\hline Loblolly pine & 898,994 & 140,432 & 114,659 & 106,733 & 107,076 & 99,380 & 94,834 & 70,484 & 65,868 & 92,828 & 6,700 \\
\hline Pond pine & 161,647 & 9,767 & 22,379 & 26,185 & 27,996 & 30,655 & 19,686 & 11,666 & 5,622 & 7,691 & - \\
\hline Virginia pine & - & - & - & - & - & - & - & - & - & - & - \\
\hline Pitch pine & - & - & - & - & - & - & - & - & - & - & - \\
\hline Table Mountain pine & - & - & - & - & - & - & - & - & - & - & - \\
\hline Spruce pine & 30,602 & 982 & 3,883 & 1,783 & 2,440 & 3,929 & 3,888 & 4,676 & 3,840 & 4,332 & 849 \\
\hline Sand pine & 494,664 & 137,640 & 138,415 & 76,386 & 59,766 & 39,437 & 25,215 & 10,421 & 5,855 & 1,529 & - \\
\hline Eastern white pine & - & - & - & - & - & - & - & - & - & - & - \\
\hline Eastern hemlock & - & - & - & - & - & - & - & - & - & - & - \\
\hline Spruce and fir & - & - & - & - & - & - & - & - & - & - & - \\
\hline Baldcypress & 556,818 & 32,022 & 57,036 & 74,136 & 79,395 & 94,655 & 71,305 & 50,597 & 34,884 & 46,585 & 16,203 \\
\hline Pondcypress & $1,840,670$ & 243,583 & 336,297 & 367,713 & 323,784 & 248,558 & 140,615 & 74,971 & 44,594 & 52,651 & 7,904 \\
\hline Cedars & 125,094 & 9,071 & 13,149 & 20,570 & 22,065 & 22,032 & 13,701 & 9,588 & 7,736 & 6,606 & 576 \\
\hline Total softwoods & $9,525,645$ & $1,593,047$ & $1,886,820$ & $1,617,098$ & $1,425,990$ & $1,185,398$ & 782,932 & 441,972 & 270,448 & 287,618 & 34,322 \\
\hline \multicolumn{12}{|l|}{ Hardwood } \\
\hline Select white oaks & 31,543 & 3,277 & 2,430 & 2,724 & 4,534 & 3,135 & 3,721 & 3,738 & 3,726 & 2,996 & 1,262 \\
\hline Select red oaks & 1,606 & - & 375 & - & - & 588 & - & - & - & 643 & - \\
\hline Chestnut oak & - & - & - & - & - & - & - & - & - & - & - \\
\hline Other white oaks & 900,564 & 46,838 & 45,405 & 52,173 & 56,617 & 60,082 & 81,925 & 70,865 & 72,588 & 224,300 & 189,771 \\
\hline Other red oaks & $1,568,968$ & 146,688 & 160,035 & 197,464 & 225,629 & 188,201 & 154,819 & 135,868 & 104,192 & 188,520 & 67,552 \\
\hline Hickory & 131,970 & 8,078 & 7,865 & 14,669 & 11,383 & 20,829 & 17,938 & 16,866 & 11,190 & 21,314 & 1,838 \\
\hline Yellow birch & - & - & - & - & - & - & - & - & - & - & - \\
\hline Hard maple & 16,159 & 1,282 & 1,742 & 1,202 & 1,891 & 3,414 & 608 & 1,937 & 625 & 3,458 & - \\
\hline Soft maple & 561,725 & 57,029 & 76,206 & 88,159 & 73,637 & 84,702 & 63,141 & 41,112 & 32,705 & 38,946 & 6,088 \\
\hline Beech & 9,686 & 307 & 419 & 584 & - & 974 & 582 & 1,044 & 1,042 & 3,850 & 884 \\
\hline Sweetgum & 584,877 & 52,447 & 62,472 & 97,375 & 93,162 & 96,083 & 65,793 & 41,913 & 31,954 & 39,946 & 3,732 \\
\hline Tupelo and blackgum & $1,658,769$ & 207,136 & 242,446 & 252,450 & 269,840 & 236,257 & 170,005 & 112,511 & 62,302 & 87,403 & 18,419 \\
\hline Ash & 419,310 & 56,464 & 58,980 & 73,409 & 64,821 & 45,630 & 41,948 & 32,083 & 19,095 & 24,974 & 1,906 \\
\hline Cottonwood & 585 & - & 283 & - & - & - & - & - & - & 302 & - \\
\hline Basswood & 15,897 & 991 & 1,991 & 1,409 & 1,227 & 2,788 & 3,189 & 1,897 & 567 & 1,838 & - \\
\hline Yellow-poplar & 85,251 & 6,265 & 6,043 & 12,687 & 10,322 & 10,190 & 10,089 & 9,825 & 8,492 & 10,158 & 1,180 \\
\hline Bay and magnolia & 982,422 & 148,834 & 157,429 & 161,595 & 155,675 & 126,316 & 85,447 & 57,276 & 33,478 & 50,175 & 6,197 \\
\hline Black cherry & 23,759 & 5,051 & 6,042 & 4,600 & 2,298 & 2,062 & 1,653 & 1,674 & 379 & - & - \\
\hline Black walnut & 232 & 232 & - & - & - & - & - & - & - & - & - \\
\hline Sycamore & 9,542 & - & 453 & - & 889 & 1,107 & 530 & 1,867 & 1,210 & 1,836 & 1,650 \\
\hline Black locust & - & - & - & - & - & - & - & - & - & - & - \\
\hline Elm & 105,949 & 9,920 & 13,252 & 15,716 & 12,041 & 15,497 & 13,230 & 9,168 & 5,382 & 10,573 & 1,170 \\
\hline Other Eastern hardwoods & 381,221 & 101,153 & 76,795 & 70,164 & 41,910 & 31,637 & 20,096 & 15,598 & 11,187 & 9,753 & 2,928 \\
\hline Total hardwoods & $7,490,035$ & 851,992 & 920,663 & $1,046,380$ & $1,025,876$ & 929,492 & 734,714 & 555,242 & 400,114 & 720,985 & 304,577 \\
\hline All species & $17,015,680$ & $2,445,039$ & $2,807,483$ & $2,663,478$ & $2,451,866$ & $2,114,890$ & $1,517,646$ & 997,214 & 670,562 & $1,008,603$ & 338,899 \\
\hline
\end{tabular}


Table 35-Volume of growing stock on timberland, by species and diameter class, Florida, 1995

\begin{tabular}{|c|c|c|c|c|c|c|c|c|c|c|c|}
\hline \multirow[b]{2}{*}{ Species } & \multirow[b]{2}{*}{$\begin{array}{c}\text { All } \\
\text { classes }\end{array}$} & \multicolumn{10}{|c|}{ Diameter class (inches at breast height) } \\
\hline & & $\begin{array}{l}5.0- \\
6.9 \\
\end{array}$ & $\begin{array}{l}7.0- \\
8.9\end{array}$ & $\begin{array}{r}9.0- \\
10.9\end{array}$ & $\begin{array}{l}11.0- \\
12.9\end{array}$ & $\begin{array}{l}13.0- \\
14.9\end{array}$ & $\begin{array}{l}15.0- \\
16.9\end{array}$ & $\begin{array}{l}17.0- \\
18.9\end{array}$ & $\begin{array}{l}19.0- \\
20.9\end{array}$ & $\begin{array}{l}21.0- \\
28.9\end{array}$ & $\begin{array}{c}29.0 \text { and } \\
\text { larger }\end{array}$ \\
\hline & \multicolumn{11}{|c|}{ Thousand cubic feet } \\
\hline \multicolumn{12}{|l|}{ Softwood } \\
\hline Longleaf pine & $1,057,491$ & 48,409 & 83,528 & 183,216 & 268,068 & 251,033 & 145,044 & 49,284 & 20,112 & 8,797 & - \\
\hline Slash pine & $4,304,676$ & 963,071 & $1,112,129$ & 756,286 & 528,928 & 386,673 & 258,617 & 155,795 & 78,675 & 62,412 & 2,090 \\
\hline Shortleaf pine & 39,139 & 1,734 & 2,721 & 1,884 & 5,010 & 7,882 & 9,133 & 3,907 & 3,262 & 3,606 & - \\
\hline Loblolly pine & 893,514 & 138,626 & 113,900 & 105,715 & 106,522 & 98,548 & 94,834 & 70,484 & 65,868 & 92,317 & 6,700 \\
\hline Pond pine & 160,074 & 9,115 & 22,163 & 25,837 & 27,996 & 30,655 & 19,329 & 11,666 & 5,622 & 7,691 & - \\
\hline Virginia pine & - & - & - & - & - & - & - & - & - & - & - \\
\hline Pitch pine & - & - & - & - & - & - & - & - & - & - & - \\
\hline Table Mountain pine & - & - & - & - & - & - & - & - & - & - & - \\
\hline Spruce pine & 30,602 & 982 & 3,883 & 1,783 & 2,440 & 3,929 & 3,888 & 4,676 & 3,840 & 4,332 & 849 \\
\hline Sand pine & 489,599 & 136,533 & 136,869 & 75,393 & 58,667 & 39,117 & 25,215 & 10,421 & 5,855 & 1,529 & - \\
\hline Eastern white pine & - & - & - & - & - & - & - & - & - & - & - \\
\hline Eastern hemlock & - & - & - & - & - & - & - & - & - & - & - \\
\hline Spruce and fir & - & - & - & - & - & - & - & - & - & - & - \\
\hline Baldcypress & 541,648 & 31,787 & 56,318 & 74,136 & 79,157 & 93,634 & 70,627 & 50,260 & 34,884 & 43,714 & 7,131 \\
\hline Pondcypress & $1,787,115$ & 232,808 & 324,255 & 358,616 & 318,798 & 244,708 & 136,624 & 72,778 & 43,597 & 48,897 & 6,034 \\
\hline Cedars & 120,576 & 8,635 & 11,662 & 19,787 & 20,634 & 22,032 & 13,320 & 9,588 & 7,736 & 6,606 & 576 \\
\hline Total softwoods & $9,424,434$ & $1,571,700$ & $1,867,428$ & $1,602,653$ & $1,416,220$ & $1,178,211$ & 776,631 & 438,859 & 269,451 & 279,901 & 23,380 \\
\hline \multicolumn{12}{|l|}{ Hardwood } \\
\hline Select white oaks & 30,372 & 2,819 & 2,278 & 2,724 & 4,084 & 3,135 & 3,721 & 3,738 & 3,726 & 2,885 & 1,262 \\
\hline Select red oaks & 1,606 & - & 375 & - & - & 588 & - & - & - & 643 & - \\
\hline Chestnut oak & - & - & - & - & - & - & - & - & - & - & - \\
\hline Other white oaks & 442,672 & 10,985 & 17,196 & 16,758 & 16,278 & 27,094 & 39,459 & 35,516 & 38,877 & 129,515 & 110,994 \\
\hline Other red oaks & $1,373,697$ & 125,485 & 139,558 & 179,309 & 207,449 & 169,803 & 136,057 & 118,820 & 90,162 & 157,159 & 49,895 \\
\hline Hickory & 121,820 & 7,098 & 6,104 & 13,278 & 10,361 & 19,523 & 15,801 & 16,622 & 10,516 & 20,679 & 1,838 \\
\hline Yellow birch & - & - & - & - & - & - & - & - & - & - & - \\
\hline Hard maple & 12,988 & 720 & 1,742 & 702 & 1,110 & 2,086 & 608 & 1,937 & 625 & 3,458 & - \\
\hline Soft maple & 445,124 & 37,323 & 57,121 & 69,996 & 59,327 & 70,499 & 50,096 & 35,763 & 27,968 & 32,836 & 4,195 \\
\hline Beech & 7,480 & 307 & 419 & 584 & - & 974 & - & 1,044 & 1,042 & 2,226 & 884 \\
\hline Sweetgum & 563,447 & 49,646 & 59,734 & 94,611 & 91,276 & 92,973 & 63,927 & 39,911 & 31,506 & 37,115 & 2,748 \\
\hline Tupelo and blackgum & $1,484,350$ & 175,010 & 213,867 & 230,301 & 244,745 & 218,045 & 157,698 & 103,574 & 54,756 & 72,398 & 13,956 \\
\hline Ash & 338,775 & 33,235 & 41,687 & 61,328 & 58,186 & 39,976 & 35,349 & 30,110 & 16,458 & 21,955 & 491 \\
\hline Cottonwood & 283 & - & 283 & - & - & - & - & - & - & - & - \\
\hline Basswood & 13,113 & 363 & 1,302 & 1,089 & 925 & 2,482 & 2,728 & 1,819 & 567 & 1,838 & - \\
\hline Yellow-poplar & 83,010 & 6,265 & 5,589 & 12,687 & 10,322 & 10,190 & 10,089 & 9,825 & 8,249 & 8,614 & 1,180 \\
\hline Bay and magnolia & 853,702 & 121,465 & 135,398 & 138,534 & 139,244 & 112,879 & 75,331 & 53,705 & 27,470 & 44,297 & 5,379 \\
\hline Black cherry & 21,296 & 4,317 & 5,663 & 4,287 & 1,889 & 2,062 & 1,653 & 1,046 & 379 & - & - \\
\hline Black wainut & - & - & - & - & - & - & - & - & - & - & - \\
\hline Sycamore & 9,542 & - & 453 & - & 889 & 1,107 & 530 & 1,867 & 1,210 & 1,836 & 1,650 \\
\hline Black locust & - & - & - & - & - & - & - & - & - & - & - \\
\hline Elm & 85,244 & 6,002 & 10,708 & 12,198 & 8,103 & 14,826 & 11,215 & 7,727 & 4,273 & 9,455 & 737 \\
\hline Other Eastern hardwoods & 53,476 & 6,933 & 7,867 & 7,250 & 8,971 & 8,899 & 6,182 & 2,106 & 3,045 & 2,223 & $=$ \\
\hline Total hardwoods & $5,941,997$ & 587,973 & 707,344 & 845,636 & 863,159 & 797,141 & 610,444 & 465,130 & 320,829 & 549,132 & 195,209 \\
\hline All species & $15,366,431$ & $2,159,673$ & $2,574,772$ & $2,448,289$ & $2,279,379$ & $1,975,352$ & $1,387,075$ & 903,989 & 590,280 & 829,033 & 218,589 \\
\hline
\end{tabular}


Table 36-Volume of sawtimber on timberland, by species and diameter class, Florida, 1995

\begin{tabular}{|c|c|c|c|c|c|c|c|c|c|}
\hline \multirow[b]{2}{*}{ Species } & \multirow[b]{2}{*}{$\begin{array}{c}\text { All } \\
\text { classes }\end{array}$} & \multicolumn{8}{|c|}{ Diameter class (inches at breast height) } \\
\hline & & $\begin{array}{c}9.0- \\
10.9 \\
\end{array}$ & $\begin{array}{l}11.0- \\
12.9 \\
\end{array}$ & $\begin{array}{l}13.0- \\
14.9 \\
\end{array}$ & $\begin{array}{l}15.0- \\
16.9 \\
\end{array}$ & $\begin{array}{l}17.0- \\
18.9 \\
\end{array}$ & $\begin{array}{l}19.0- \\
20.9 \\
\end{array}$ & $\begin{array}{l}21.0- \\
28.9 \\
\end{array}$ & $\begin{array}{c}29.0 \text { and } \\
\text { larger }\end{array}$ \\
\hline & \multicolumn{9}{|c|}{ Thousand board feet } \\
\hline \multicolumn{10}{|l|}{ Softwood } \\
\hline Longleaf pine & $4,709,463$ & 749,730 & $1,292,944$ & $1,346,166$ & 835,756 & 299,038 & 127,503 & 58,326 & - \\
\hline Slash pine & $10,599,513$ & $2,770,781$ & $2,427,358$ & $2,029,626$ & $1,480,234$ & 949,718 & 503,570 & 422,752 & 15,474 \\
\hline Shortleaf pine & 188,709 & 6,974 & 23,098 & 40,629 & 50,947 & 22,978 & 20,187 & 23,896 & - \\
\hline Loblolly pine & $3,416,601$ & 378,801 & 480,139 & 511,735 & 533,075 & 424,379 & 417,221 & 622,294 & 48,957 \\
\hline Pond pine & 646,053 & 99,253 & 127,925 & 157,491 & 107,824 & 68,750 & 34,811 & 49,999 & - \\
\hline Virginia pine & - & - & - & - & - & - & - & - & - \\
\hline Pitch pine & - & - & - & - & - & - & - & - & - \\
\hline Table Mountain pine & - & - & - & - & - & - & - & - & - \\
\hline Spruce pine & 144,622 & 7,334 & 11,599 & 20,815 & 21,451 & 27,143 & 23,169 & 27,461 & 5,650 \\
\hline Sand pine & $1,026,186$ & 290,592 & 274,179 & 206,723 & 144,537 & 63,143 & 36,987 & 10,025 & - \\
\hline Eastern white pine & - & - & - & - & - & - & - & - & - \\
\hline Eastern hemlock & - & - & - & - & - & - & - & - & - \\
\hline Spruce and fir & - & - & - & - & - & - & - & - & - \\
\hline Baldcypress & $2,013,648$ & 223,840 & 299,148 & 408,030 & 338,814 & 257,883 & 187,702 & 251,973 & 46,258 \\
\hline Pondcypress & $5,060,408$ & $1,126,019$ & $1,245,455$ & $1,088,991$ & 665,219 & 377,228 & 236,734 & 282,337 & 38,425 \\
\hline Cedars & 506,336 & 77,445 & 93,980 & 113,612 & 73,146 & 55,402 & 46,709 & 42,161 & 3,881 \\
\hline Total softwoods & $28,311,539$ & $5,730,769$ & $6,275,825$ & $5,923,818$ & $4,251,003$ & $2,545,662$ & $1,634,593$ & $1,791,224$ & 158,645 \\
\hline \multicolumn{10}{|l|}{ Hardwood } \\
\hline Select white oaks & 104,920 & - & 13,932 & 12,506 & 16,525 & 17,970 & 19,012 & 16,580 & 8,395 \\
\hline Select red oaks & 6,074 & - & - & 2,628 & - & - & - & 3,446 & - \\
\hline Chestnut oak & - & - & - & - & - & - & - & - & - \\
\hline Other white oaks & $1,979,374$ & - & 56,387 & 107,149 & 169,771 & 163,302 & 187,360 & 670,284 & 625,121 \\
\hline Other red oaks & $4,480,354$ & - & 793,328 & 737,889 & 650,162 & 603,536 & 481,570 & 900,692 & 313,177 \\
\hline Hickory & 447,621 & - & 35,263 & 79,763 & 71,740 & 80,977 & 54,334 & 114,338 & 11,206 \\
\hline Yellow birch & - & - & - & - & - & - & - & - & - \\
\hline Hard maple & 44,337 & - & 3,694 & 8,097 & 2,678 & 8,901 & 3,036 & 17,931 & - \\
\hline Soft maple & $1,162,297$ & - & 193,894 & 268,769 & 212,000 & 162,062 & 133,840 & 168,190 & 23,542 \\
\hline Beech & 25,002 & - & - & 3,707 & - & 4,127 & 4,227 & 9,193 & 3,748 \\
\hline Sweetgum & $1,656,633$ & - & 325,874 & 397,349 & 307,831 & 209,350 & 175,097 & 222,811 & 18,321 \\
\hline Tupelo and blackgum & $3,591,270$ & - & 797,699 & 849,023 & 692,033 & 493,474 & 275,745 & 397,516 & 85,780 \\
\hline Ash & 840,785 & - & 190,389 & 153,221 & 152,742 & 141,248 & 82,575 & 117,607 & 3,003 \\
\hline Cottonwood & - & - & - & - & - & - & - & - & - \\
\hline Basswood & 45,289 & - & 3,205 & 9,716 & 11,712 & 8,444 & 2,757 & 9,455 & - \\
\hline Yellow-poplar & 292,057 & - & 37,610 & 43,332 & 49,677 & 52,720 & 47,106 & 53,492 & 8,120 \\
\hline Bay and magnolia & $1,852,308$ & - & 462,093 & 434,959 & 317,981 & 246,727 & 131,470 & 228,384 & 30,694 \\
\hline Black cherry & 29,701 & - & 6,528 & 8,528 & 7,572 & 5,104 & 1,969 & - & - \\
\hline Black walnut & - & - & - & - & - & - & - & - & - \\
\hline Sycamore & 43,748 & - & 3,039 & 4,150 & 2,231 & 8,656 & 5,981 & 9,919 & 9,772 \\
\hline Black locust & - & - & - & - & - & - & - & - & - \\
\hline Elm & 241,058 & - & 27,757 & 58,139 & 47,940 & 35,219 & 20,342 & 47,609 & 4,052 \\
\hline Other Eastern hardwoods & 132,863 & - & 30,971 & 35,890 & 27,736 & 9,991 & 15,912 & 12,363 & - \\
\hline Total hardwoods & $16,975,691$ & - & $2,981,663$ & $3,214,815$ & $2,740,331$ & $2,251,808$ & $1,642,333$ & $2,999,810$ & $1,144,931$ \\
\hline All species & $45,287,230$ & $5,730,769$ & $9,257,488$ & $9,138,633$ & $6,991,334$ & $4,797,470$ & $3,276,926$ & $4,791,034$ & $1,303,576$ \\
\hline
\end{tabular}


Table 37-Volume of sawtimber on timberland, by species, size class, and tree grade, Florida, 1995

\begin{tabular}{|c|c|c|c|c|c|c|c|c|c|c|}
\hline \multirow[b]{3}{*}{ Species } & \multicolumn{5}{|c|}{ All size classes } & \multicolumn{5}{|c|}{ Trees 15.0 inches d.b.h. and larger } \\
\hline & \multirow{2}{*}{$\begin{array}{c}\text { All } \\
\text { grades }\end{array}$} & \multicolumn{4}{|c|}{ Tree grade } & \multirow{2}{*}{$\begin{array}{c}\text { All } \\
\text { grades }\end{array}$} & \multicolumn{4}{|c|}{ Tree grade } \\
\hline & & 1 & 2 & 3 & 4 & & 1 & 2 & 3 & 4 \\
\hline & \multicolumn{10}{|c|}{ Thousand board feet } \\
\hline \multicolumn{11}{|l|}{ Softwood } \\
\hline Yellow pines ${ }^{a}$ & $20,731,147$ & $5,660,914$ & $5,546,265$ & $9,523,968$ & - & $7,477,255$ & $3,082,412$ & $1,987,942$ & $2,406,901$ & - \\
\hline Eastern white pine $e^{b}$ & - & - & - & - & - & - & - & - & - & - \\
\hline Spruce and $\mathrm{fir}^{b}$ & - & - & - & - & - & - & - & - & - & - \\
\hline Cypress $^{c}$ & $7,074,056$ & $1,229,713$ & $2,127,296$ & $3,693,329$ & 23,718 & $2,682,573$ & $1,229,713$ & $1,080,307$ & 367,267 & 5,286 \\
\hline Other Eastern softwoods ${ }^{b}$ & 506,336 & 107,291 & 167,663 & 204,829 & 26,553 & 221,299 & 76,079 & 75,460 & 66,532 & 3,228 \\
\hline Total & $28,311,539$ & $6,997,918$ & $7,841,224$ & $13,422,126$ & 50,271 & $10,381,127$ & $4,388,204$ & $3,143,709$ & $2,840,700$ & 8,514 \\
\hline \multicolumn{11}{|l|}{ Hardwood ${ }^{c}$} \\
\hline Select white and red oaks & 110,994 & 13,838 & 47,802 & 42,165 & 7,189 & 81,928 & 13,838 & 47,802 & 17,181 & 3,107 \\
\hline Other white and red oaks & $6,459,728$ & 745,961 & $1,783,675$ & $3,355,877$ & 574,215 & $4,764,975$ & 745,961 & $1,620,830$ & $2,086,031$ & 312,153 \\
\hline Hickory & 447,621 & 76,831 & 177,849 & 182,413 & 10,528 & 332,595 & 76,831 & 150,456 & 94,780 & 10,528 \\
\hline Yellow birch & - & - & - & - & - & - & - & - & - & - \\
\hline Hard maple & 44,337 & - & 13,161 & 18,414 & 12,762 & 32,546 & - & 10,886 & 14,720 & 6,940 \\
\hline Sweetgum & $1,656,633$ & 199,259 & 656,253 & 736,118 & 65,003 & 933,410 & 199,259 & 480,177 & 217,671 & 36,303 \\
\hline Ash, walnut, and black cherry & 870,486 & 62,710 & 274,949 & 504,396 & 28,431 & 511,820 & 62,710 & 224,301 & 208,858 & 15,951 \\
\hline Yellow-poplar & 292,057 & 47,007 & 129,609 & 107,783 & 7,658 & 211,115 & 47,007 & 115,618 & 48,490 & - \\
\hline Other Eastern hardwoods & $7,093,835$ & 636,585 & $2,204,479$ & $3,977,131$ & 275,640 & $3,910,824$ & 636,585 & $1,621,312$ & $1,476,870$ & 176,057 \\
\hline Total & $16,975,691$ & $1,782,191$ & $5,287,777$ & $8,924,297$ & 981,426 & $10,779,213$ & $1,782,191$ & $4,271,382$ & $4,164,601$ & 561,039 \\
\hline All species & $45,287,230$ & $8,780,109$ & $13,129,001$ & $22,346,423$ & $1,031,697$ & $21,160,340$ & $6,170,395$ & $7,415,091$ & $7,005,301$ & 569,553 \\
\hline
\end{tabular}

For yellow pines, tree grade is based on "Southern Pine Tree Grades for Yard and Structural Lumber," Research Paper SE-40, published by the Southeastern Forest Experiment Station, Asheville, NC, 1968.

Tree grade 4 does not apply to yellow pine.

Tree grade 4 does not apply to yellow pine.

For other softwoods (excluding cypress), tree grade is based on "Tree Grades for Eastern White Pine," Research Paper NE-214, published by the Northeastern Forest Experiment Station, Radnor, PA, 1971.

c For hardwoods and cypress, tree grades 1, 2, and 3 are based on "Hardwood Tree Grades for Factory Lumber," Research Paper NE-333, published by the Northeastern Forest Experiment Station, Radnor, PA, 1976.

Grade 4 trees are sawtimber trees not qualifying as tree grades 1,2, or 3. The butt log of these trees qualify as construction (tie and timber) logs based on "A Guide to Hardwood Log Grading (revised)," General
Technical Report NE-1, published by the Northeastern Forest Experiment Station, Radnor, PA, 1971. 
Table 38-Cubic volume in the merchantable saw-log portion of sawtimber trees on timberland, by species and diameter class, Florida, 1995

\begin{tabular}{|c|c|c|c|c|c|c|c|c|c|}
\hline \multirow[b]{2}{*}{ Species } & \multirow[b]{2}{*}{$\begin{array}{c}\text { All } \\
\text { classes }\end{array}$} & \multicolumn{8}{|c|}{ Diameter class (inches at breast height) } \\
\hline & & $\begin{array}{c}9.0- \\
10.9\end{array}$ & $\begin{array}{l}11.0- \\
12.9\end{array}$ & $\begin{array}{l}13.0- \\
14.9\end{array}$ & $\begin{array}{l}15.0- \\
16.9\end{array}$ & $\begin{array}{l}17.0- \\
18.9\end{array}$ & $\begin{array}{l}19.0 \\
20.9\end{array}$ & $\begin{array}{l}21.0- \\
28.9\end{array}$ & $\begin{array}{c}29.0 \text { and } \\
\text { larger }\end{array}$ \\
\hline & \multicolumn{9}{|c|}{ Thousand cubic feet } \\
\hline Longleaf pine & 854,166 & 152,308 & 245,480 & 238,899 & 140,632 & 48,288 & 19,849 & 8,710 & - \\
\hline Slash pine & $1,991,009$ & 597,446 & 478,970 & 367,748 & 251,572 & 153,518 & 77,894 & 61,792 & 2,069 \\
\hline Shortleaf pine & 33,127 & 1,494 & 4,584 & 7,511 & 8,893 & 3,848 & 3,228 & 3,569 & - \\
\hline Loblolly pine & 595,730 & 82,201 & 95,684 & 93,417 & 91,891 & 69,312 & 65,205 & 91,387 & 6,633 \\
\hline Pitch pine & - & - & - & - & - & - & - & - & - \\
\hline Table Mountain pine & - & - & - & - & - & - & - & - & - \\
\hline Spruce pine & 24,703 & 1,445 & 2,218 & 3,756 & 3,766 & 4,590 & 3,799 & 4,289 & 840 \\
\hline Sand pine & 192,348 & 60,477 & 53,035 & 37,013 & 24,366 & 10,182 & 5,762 & 1,513 & - \\
\hline Eastern white pine & - & - & - & - & - & - & - & - & - \\
\hline Eastern hemlock & - & - & - & - & - & - & - & - & - \\
\hline Spruce and fir & - & - & - & - & - & - & - & - & - \\
\hline \multicolumn{10}{|l|}{ Hardwood } \\
\hline Select white oaks & 19,538 & - & 2,856 & 2,538 & 3,256 & 3,397 & 3,465 & 2,777 & 1,249 \\
\hline Select red oaks & 1,096 & - & - & 496 & - & - & - & 600 & - \\
\hline Chestnut oak & - & - & - & - & - & - & - & - & - \\
\hline Other white oaks & 360,953 & - & 11,961 & 22,267 & 34,261 & 31,786 & 35,404 & 120,401 & 104,873 \\
\hline Other red oaks & 792,921 & - & 152,297 & 139,759 & 118,454 & 106,492 & 82,339 & 146,254 & 47,326 \\
\hline Hickory & 83,204 & - & 7,385 & 16,138 & 13,836 & 14,999 & 9,686 & 19,400 & 1,760 \\
\hline Yellow birch & - & - & - & - & - & - & - & - & - \\
\hline Hard maple & 8,522 & - & 752 & 1,680 & 534 & 1,738 & 574 & 3,244 & - \\
\hline Soft maple & 229,824 & - & 40,573 & 55,969 & 42,583 & 31,448 & 25,152 & 30,160 & 3,939 \\
\hline Beech & 5,474 & - & - & 795 & - & 909 & 933 & 2,021 & 816 \\
\hline Sweetgum & 302,896 & - & 63,967 & 76,675 & 56,809 & 36,938 & 29,819 & 35,968 & 2,720 \\
\hline Tupelo and blackgum & 714,192 & - & 174,325 & 178,069 & 137,141 & 93,218 & 50,238 & 67,831 & 13,370 \\
\hline Ash & 167,788 & - & 40,812 & 32,312 & 30,798 & 27,280 & 15,298 & 20,809 & 479 \\
\hline Cottonwood & - & - & - & - & - & - & - & - & - \\
\hline Basswood & 8,975 & - & 682 & 2,036 & 2,374 & 1,642 & 521 & 1,720 & - \\
\hline Yellow-poplar & 50,921 & - & 7,295 & 8,254 & 8,954 & 9,082 & 7,807 & 8,361 & 1,168 \\
\hline Bay and magnolia & 379,244 & - & 96,507 & 92,720 & 66,485 & 49,512 & 25,889 & 42,816 & 5,315 \\
\hline Black cherry & 5,803 & - & 1,379 & 1,707 & 1,438 & 933 & 346 & - & - \\
\hline Black walnut & - & - & - & - & - & - & - & - & - \\
\hline Sycamore & 7,917 & - & 621 & 842 & 441 & 1,637 & 1,093 & 1,711 & 1,572 \\
\hline Black locust & - & - & - & - & - & - & - & - & - \\
\hline Elm & 46,971 & - & 5,695 & 11,894 & 9,520 & 6,774 & 3,813 & 8,591 & 684 \\
\hline Other Eastern hardwoods & 24,364 & - & 6,024 & 6,867 & 5,101 & 1,845 & 2,595 & 1,932 & $=$ \\
\hline Total hardwoods & $3,210,603$ & - & 613,131 & 651,018 & 531,985 & 419,630 & 294,972 & 514,596 & 185,271 \\
\hline All species & $8,584,865$ & $1,261,954$ & $1,883,351$ & $1,758,216$ & $1,278,506$ & 846,699 & 558,770 & 789,174 & 208,195 \\
\hline
\end{tabular}


Table 39-Total volume of live trees on timberland, by species and diameter class, Florida, 1995

\begin{tabular}{|c|c|c|c|c|c|c|c|c|c|c|c|c|}
\hline \multirow[b]{2}{*}{ Species } & \multirow[b]{2}{*}{$\begin{array}{c}\text { All } \\
\text { classes }\end{array}$} & \multicolumn{11}{|c|}{ Diameter class (inches at breast height) } \\
\hline & & $\begin{array}{l}1.0- \\
2.9 \\
\end{array}$ & $\begin{array}{l}3.0- \\
4.9 \\
\end{array}$ & $\begin{array}{l}5.0- \\
6.9 \\
\end{array}$ & $\begin{array}{r}7.0- \\
8.9 \\
\end{array}$ & $\begin{array}{r}9.0- \\
10.9 \\
\end{array}$ & $\begin{array}{l}11.0- \\
12.9 \\
\end{array}$ & $\begin{array}{l}13.0- \\
14.9 \\
\end{array}$ & $\begin{array}{l}15.0- \\
16.9 \\
\end{array}$ & $\begin{array}{l}17.0- \\
18.9 \\
\end{array}$ & $\begin{array}{l}19.0- \\
20.9 \\
\end{array}$ & $\begin{array}{r}21.0- \\
28.9 \\
\end{array}$ \\
\hline & & \multicolumn{11}{|c|}{ Thousand cubic feet } \\
\hline \multicolumn{13}{|l|}{ Softwood } \\
\hline Longleaf pine & $1,277,909$ & 16,545 & 40,205 & 68,652 & 100,632 & 211,755 & 305,837 & 283,532 & 163,031 & 55,128 & 22,448 & 10,144 \\
\hline Slash pine & $5,987,966$ & 139,008 & 596,533 & $1,364,846$ & $1,348,345$ & 877,744 & 601,433 & 435,837 & 290,172 & 174,454 & 87,580 & 69,695 \\
\hline Shortleaf pine & 45,737 & 227 & 265 & 2,365 & 3,669 & 2,187 & 5,714 & 8,924 & 10,297 & 4,394 & 3,659 & 4,036 \\
\hline Loblolly pine & $1,208,633$ & 28,174 & 107,153 & 202,047 & 140,358 & 124,554 & 122,538 & 112,612 & 106,946 & 79,180 & 73,817 & 103,779 \\
\hline Pond pine & 195,961 & 1,137 & 6,444 & 13,091 & 26,928 & 30,481 & 32,189 & 35,014 & 22,383 & 13,226 & 6,375 & 8,693 \\
\hline Virginia pine & - & - & - & - & - & - & - & - & - & - & - & - \\
\hline Pitch pine & - & - & - & - & - & - & - & - & - & - & - & - \\
\hline Table Mountain pine & - & - & - & - & - & - & - & - & - & - & - & - \\
\hline Spruce pine & 36,208 & 672 & 470 & 1,330 & 4,652 & 2,067 & 2,788 & 4,452 & 4,391 & 5,264 & 4,315 & 4,857 \\
\hline Sand pine & 796,887 & 48,584 & 123,506 & 202,636 & 170,537 & 89,610 & 68,681 & 44,840 & 28,475 & 11,728 & 6,576 & 1,714 \\
\hline Eastern white pine & - & - & - & - & - & - & - & - & - & - & - & - \\
\hline Eastern hemiock & - & - & - & - & - & - & - & - & - & - & - & - \\
\hline Spruce and fir & - & - & - & - & - & - & - & - & - & - & - & - \\
\hline Baldcypress & 717,393 & 5,325 & 21,192 & 47,430 & 73,497 & 91,872 & 96,836 & 114,515 & 85,964 & 60,739 & 41,785 & 56,089 \\
\hline Pondcypress & $2,795,319$ & 84,277 & 213,515 & 409,528 & 466,569 & 483,704 & 416,375 & 315,953 & 177,768 & 94,684 & 56,140 & 66,639 \\
\hline Cedars & 162,173 & 4,562 & 7,800 & 12,704 & 16,351 & 24,567 & 25,964 & 25,740 & 15,970 & 11,172 & 9,009 & 7,669 \\
\hline Total softwoods & $13,224,186$ & 328,511 & $1,117,083$ & $2,324,629$ & $2,351,538$ & $1,938,541$ & $1,678,355$ & $1,381,419$ & 905,397 & 509,969 & 311,704 & 333,315 \\
\hline \multicolumn{13}{|l|}{ Hardwood } \\
\hline Select white oaks & 43,804 & 800 & 2,758 & 4,774 & 3,205 & 3,458 & 5,678 & 3,924 & 4,613 & 4,617 & 4,590 & 3,810 \\
\hline Select red oaks & 2,084 & 83 & - & - & 489 & - & - & 725 & - & - & - & 787 \\
\hline Chestnut oak & - & - & - & - & - & - & - & - & - & - & - & - \\
\hline Other white oaks & $1,222,010$ & 28,275 & 56,969 & 82,380 & 62,573 & 67,160 & 70,946 & 74,327 & 100,328 & 86,777 & 88,678 & 272,427 \\
\hline Other red oaks & $2,324,043$ & 122,831 & 161,831 & 227,327 & 215,027 & 254,628 & 286,032 & 237,440 & 194,728 & 170,351 & 130,330 & 237,246 \\
\hline Hickory & 171,910 & 3,615 & 4,103 & 12,657 & 10,358 & 18,407 & 14,002 & 25,315 & 21,724 & 20,359 & 13,450 & 25,670 \\
\hline Yellow birch & - & - & $\overline{-}$ & - & - & - & - & - & $\overline{-}$ & - & - & - \\
\hline Hard maple & 21,825 & 839 & 776 & 1,998 & 2,248 & 1,494 & 2,347 & 4,154 & 736 & 2,347 & 747 & 4,139 \\
\hline Soft maple & 816,336 & 43,521 & 76,986 & 82,076 & 96,452 & 108,024 & 89,109 & 102,092 & 75,914 & 49,082 & 39,140 & 46,565 \\
\hline Beech & 13,055 & 240 & 441 & 428 & 552 & 741 & - & 1,206 & 746 & 1,286 & 1,300 & 5,034 \\
\hline Sweetgum & 784,953 & 31,881 & 55,823 & 77,176 & 77,735 & 115,110 & 107,967 & 110,316 & 75,041 & 47,761 & 36,265 & 45,484 \\
\hline Tupelo and blackgum & $2,479,349$ & 115,069 & 240,780 & 312,172 & 315,548 & 315,434 & 333,264 & 289,837 & 208,273 & 138,702 & 77,450 & 109,285 \\
\hline Ash & 618,528 & 40,743 & 70,156 & 80,625 & 72,925 & 87,035 & 75,555 & 53,091 & 48,446 & 36,764 & 22,131 & 28,743 \\
\hline Cottonwood & 835 & 90 & - & - & 353 & - & - & - & - & - & - & 392 \\
\hline Basswood & 20,748 & 852 & 1,318 & 1,294 & 2,375 & 1,646 & 1,423 & 3,228 & 3,650 & 2,207 & 646 & 2,109 \\
\hline Yellow-poplar & 104,347 & 1,805 & 4,321 & 8,383 & 7,154 & 14,635 & 11,759 & 11,530 & 11,360 & 11,043 & 9,560 & 11,475 \\
\hline Bay and magnolia & $1,486,548$ & 83,334 & 177,597 & 218,627 & 198,884 & 196,498 & 186,295 & 150,020 & 101,267 & 67,617 & 39,679 & 59,464 \\
\hline Black cherry & 44,511 & 7,068 & 8,133 & 6,874 & 7,437 & 5,500 & 2,722 & 2,426 & 1,939 & 1,961 & 451 & - \\
\hline Black walnut & 481 & 18 & 145 & 318 & - & - & - & - & - & - & - & - \\
\hline Sycamore & 11,563 & - & 471 & - & 562 & - & 1,037 & 1,289 & 615 & 2,170 & 1,400 & 2,119 \\
\hline Black locust & - & - & - & - & - & - & - & - & - & $\overline{-}$ & - & - \\
\hline Elm & 150,741 & 5,453 & 16,383 & 14,201 & 16,594 & 18,987 & 14,388 & 18,301 & 15,545 & 10,813 & 6,306 & 12,359 \\
\hline Other Eastern hardwoods & 844,330 & 157,309 & 178,356 & 152,673 & 100,858 & 88,627 & 52,343 & 39,110 & 25,115 & 19,498 & 14,056 & 12,217 \\
\hline Total hardwoods & $11,162,001$ & 643,826 & $1,057,347$ & $1,283,983$ & $1,191,329$ & $1,297,384$ & $1,254,867$ & $1,128,331$ & 890,040 & 673,355 & 486,179 & 879,325 \\
\hline All species & $24,386,187$ & 972,337 & $2,174,430$ & $3,608,612$ & $3,542,867$ & $3,235,925$ & $2,933,222$ & $2,509,750$ & $1,795,437$ & $1,183,324$ & 797,883 & $1,212,640$ \\
\hline
\end{tabular}


Table 40-Green weight of forest biomass on timberland, by species and diameter class, Florida, 1995

\begin{tabular}{|c|c|c|c|c|c|c|c|c|c|c|c|c|c|}
\hline \multirow[b]{2}{*}{ Species } & \multirow[b]{2}{*}{$\begin{array}{c}\text { All } \\
\text { classes }\end{array}$} & \multicolumn{12}{|c|}{ Diameter class (inches at breast height) } \\
\hline & & $\begin{array}{l}1.0- \\
2.9\end{array}$ & $\begin{array}{l}3.0- \\
4.9\end{array}$ & $\begin{array}{l}5.0- \\
6.9\end{array}$ & $\begin{array}{l}7.0- \\
8.9\end{array}$ & $\begin{array}{r}9.0- \\
10.9\end{array}$ & $\begin{array}{l}11.0- \\
12.9\end{array}$ & $\begin{array}{l}13.0- \\
14.9\end{array}$ & $\begin{array}{l}15.0- \\
16.9\end{array}$ & $\begin{array}{l}17.0- \\
18.9\end{array}$ & $\begin{array}{l}19.0- \\
20.9\end{array}$ & $\begin{array}{r}21.0- \\
28.9\end{array}$ & $\begin{array}{c}29.0 \text { and } \\
\text { larger }\end{array}$ \\
\hline & \multicolumn{13}{|c|}{ Hundred thousand pounds } \\
\hline \multicolumn{14}{|l|}{ Softwood } \\
\hline Longleaf pine & $1,000,186$ & 13,317 & 34,287 & 48,239 & 76,238 & 163,671 & 240,230 & 224,374 & 129,673 & 43,956 & 18,071 & 8,130 & - \\
\hline Slash pine & $4,636,747$ & 102,618 & 552,756 & 988,598 & $1,029,887$ & 676,693 & 465,710 & 337,639 & 224,571 & 135,075 & 67,736 & 53,683 & 1,781 \\
\hline Shortleaf pine & 32,443 & 92 & 164 & 1,435 & 2,532 & 1,555 & 4,103 & 6,373 & 7,411 & 3,174 & 2,644 & 2,960 & - \\
\hline Loblolly pine & 857,178 & 14,343 & 63,342 & 142,020 & 104,775 & 91,640 & 89,979 & 82,354 & 77,493 & 57,526 & 53,503 & 74,917 & 5,286 \\
\hline Pond pine & 138,463 & 633 & 3,628 & 9,118 & 19,191 & 21,848 & 22,969 & 25,110 & 15,925 & 9,425 & 4,484 & 6,132 & - \\
\hline Virginia pine & - & - & - & - & - & - & - & - & - & - & - & - & - \\
\hline Pitch pine & - & - & - & - & - & - & - & - & - & - & - & - & - \\
\hline Table Mountain pine & - & - & - & - & - & - & - & - & - & - & - & - & - \\
\hline Spruce pine & 25,160 & 497 & 436 & 725 & 3,048 & 1,420 & 1,982 & 3,107 & 3,044 & 3,731 & 3,070 & 3,437 & 663 \\
\hline Sand pine & 543,614 & 36,794 & 101,140 & 121,729 & 111,405 & 60,898 & 47,279 & 30,806 & 19,815 & 8,031 & 4,558 & 1,159 & - \\
\hline Eastern white pine & - & - & - & - & - & - & - & - & - & - & - & - & - \\
\hline Eastern hemlock & - & - & - & - & - & - & - & - & - & - & - & - & - \\
\hline Spruce and fir & - & - & - & - & - & - & - & - & - & - & - & - & - \\
\hline Baldcypress & 523,301 & 2,929 & 13,387 & 23,870 & 45,173 & 63,054 & 70,996 & 87,146 & 67,221 & 48,683 & 33,956 & 47,100 & 19,786 \\
\hline Pondcypress & $1,710,742$ & 45,977 & 133,945 & 179,019 & 254,977 & 296,634 & 277,085 & 220,980 & 128,836 & 70,385 & 42,582 & 51,965 & 8,357 \\
\hline Cedars & 125,935 & 3,273 & 5,066 & 9,193 & 12,417 & 18,825 & 20,663 & 20,656 & 13,023 & 8,966 & 7,169 & 6,129 & 555 \\
\hline Total softwoods & $9,593,769$ & 220,473 & 908,151 & $1,523,946$ & $1,659,643$ & $1,396,238$ & $1,240,996$ & $1,038,545$ & 687,012 & 388,952 & 237,773 & 255,612 & 36,428 \\
\hline \multicolumn{14}{|l|}{ Hardwood } \\
\hline Select white oaks & 35,970 & 644 & 2,081 & 3,310 & 2,638 & 2,809 & 4,820 & 3,273 & 3,757 & 3,876 & 3,904 & 3,429 & 1,429 \\
\hline Select red oaks & 1,764 & 71 & - & - & 369 & - & - & 627 & - & - & - & 697 & - \\
\hline Chestnut oak & - & - & - & - & - & - & - & - & - & - & - & - & - \\
\hline Other white oaks & $1,106,709$ & 21,334 & 42,584 & 46,057 & 48,774 & 57,706 & 64,280 & 68,655 & 94,698 & 82,775 & 84,418 & 265,002 & 230,426 \\
\hline Other red oaks & $1,867,360$ & 108,172 & 122,804 & 170,176 & 176,433 & 206,409 & 232,135 & 193,618 & 158,956 & 138,468 & 105,806 & 187,455 & 66,928 \\
\hline Hickory & 142,185 & 3,145 & 3,638 & 8,863 & 8,109 & 14,605 & 11,331 & 20,996 & 18,304 & 17,267 & 11,449 & 22,427 & 2,051 \\
\hline Yellow birch & - & - & - & - & - & - & - & - & - & - & - & - & - \\
\hline Hard maple & 18,956 & 682 & 675 & 1,363 & 1,901 & 1,297 & 1,985 & 3,730 & 703 & 2,089 & 673 & 3,858 & - \\
\hline Soft maple & 598,857 & 33,047 & 54,816 & 57,470 & 73,580 & 81,049 & 65,720 & 75,381 & 55,806 & 35,738 & 27,912 & 33,227 & 5,111 \\
\hline Beech & 10,934 & 192 & 365 & 310 & 411 & 522 & - & 1,036 & 776 & 1,096 & 1,118 & 4,249 & 859 \\
\hline Sweetgum & 569,001 & 21,471 & 37,048 & 49,526 & 55,032 & 83,040 & 79,453 & 82,514 & 57,258 & 36,524 & 27,884 & 35,660 & 3,591 \\
\hline Tupelo and blackgum & $1,621,754$ & 75,992 & 160,281 & 156,769 & 190,827 & 200,980 & 221,743 & 201,320 & 148,470 & 102,333 & 59,312 & 85,017 & 18,710 \\
\hline Ash & 399,287 & 25,137 & 44,413 & 62,860 & 52,877 & 57,040 & 46,797 & 31,823 & 28,456 & 20,974 & 12,378 & 15,349 & 1,183 \\
\hline Cottonwood & 622 & 61 & - & - & 257 & - & - & - & - & - & - & 304 & - \\
\hline Basswood & 14,366 & 586 & 931 & 747 & 1,630 & 1,076 & 1,054 & 2,267 & 2,580 & 1,549 & 470 & 1,476 & - \\
\hline Yellow-poplar & 73,797 & 1,326 & 2,903 & 4,933 & 4,860 & 10,338 & 8,396 & 8,342 & 8,131 & 8,064 & 7,031 & 8,454 & 1,019 \\
\hline Bay and magnolia & 925,266 & 52,123 & 106,936 & 117,178 & 122,037 & 123,617 & 119,761 & 98,820 & 67,299 & 45,302 & 26,806 & 40,386 & 5,001 \\
\hline Black cherry & 28,362 & 3,594 & 5,429 & 4,075 & 4,895 & 3,672 & 1,859 & 1,676 & 1,414 & 1,406 & 342 & - & - \\
\hline Black walnut & 429 & 11 & 127 & 291 & - & - & - & - & - & - & - & - & - \\
\hline Sycamore & 8,590 & - & 310 & - & 323 & - & 756 & 913 & 459 & 1,615 & 1,072 & 1,656 & 1,486 \\
\hline Black locust & - & - & - & - & - & - & - & - & - & - & - & - & - \\
\hline Elm & 101,077 & 4,007 & 11,423 & 9,002 & 10,966 & 12,585 & 9,645 & 12,362 & 10,141 & 7,434 & 4,260 & 8,291 & 961 \\
\hline Other Eastern hardwoods & 725,218 & 136,335 & 167,986 & 131,759 & 89,796 & 77,482 & 41,739 & 31,255 & 17,896 & 13,162 & 8,624 & 7,336 & 1,848 \\
\hline Total hardwoods & $8,250,504$ & 487,930 & 764,750 & 824,689 & 845,715 & 934,227 & 911,474 & 838,608 & 675,104 & 519,672 & 383,459 & 724,273 & 340,603 \\
\hline All species & $17,844,273$ & 708,403 & $1,672,901$ & $2,348,635$ & $2,505,358$ & $2,330,465$ & $2,152,470$ & $1,877,153$ & $1,362,116$ & 908,624 & 621,232 & 979,885 & 377,031 \\
\hline
\end{tabular}


Table 41-Average net annual growth and removals of live timber and growing stock on timberland, by species, Florida, 1987-1994

\begin{tabular}{|c|c|c|c|c|}
\hline \multirow[b]{2}{*}{ Species } & \multicolumn{2}{|c|}{ Live timber $^{a}$} & \multicolumn{2}{|c|}{ Growing stock } \\
\hline & $\begin{array}{c}\text { Net } \\
\text { annual } \\
\text { growth }\end{array}$ & $\begin{array}{c}\text { Annual } \\
\text { timber } \\
\text { removals }\end{array}$ & $\begin{array}{l}\text { Net } \\
\text { annual } \\
\text { growth }\end{array}$ & $\begin{array}{c}\text { Annual } \\
\text { timber } \\
\text { removals }\end{array}$ \\
\hline & \multicolumn{4}{|c|}{ Thousand cubic feet } \\
\hline \multicolumn{5}{|l|}{ Softwood } \\
\hline Yellow pines & 490,008 & 433,155 & 488,724 & 431,436 \\
\hline Eastern white pine & - & - & - & - \\
\hline Spruce and fir & - & - & - & - \\
\hline Cypress & 40,236 & 41,724 & 39,919 & 40,754 \\
\hline Other Eastern softwoods & 3,122 & 476 & 3,110 & 443 \\
\hline Total softwoods & 533,366 & 475,355 & 531,753 & 472,633 \\
\hline \multicolumn{5}{|l|}{ Hardwood } \\
\hline Select white and red oaks & 962 & 1,199 & 934 & 1,199 \\
\hline Other white and red oaks & 80,390 & 48,997 & 68,010 & 39,534 \\
\hline Hickory & 3,371 & 3,165 & 3,360 & 3,062 \\
\hline Yellow birch & - & - & - & - \\
\hline Hard maple & 353 & 475 & 475 & 379 \\
\hline Sweetgum & 15,298 & 12,098 & 15,051 & 11,403 \\
\hline Ash, walnut, and black cherry & 6,230 & 3,587 & 5,884 & 3,167 \\
\hline Yellow-poplar & 3,318 & 1,258 & 3,262 & 1,147 \\
\hline Tupelo and blackgum & 27,414 & 15,285 & 26,238 & 13,621 \\
\hline Bay and magnolia & 25,368 & 8,839 & 23,585 & 7,881 \\
\hline Other Eastern hardwoods & 20,807 & 13,824 & 14,082 & 6,693 \\
\hline Total hardwoods & 183,511 & 108,727 & 160,881 & 88,086 \\
\hline All species & 716,877 & 584,082 & 692,634 & 560,719 \\
\hline
\end{tabular}

${ }^{a}$ Merchantable portion only. 
Table 42-Average net annual growth and removals of sawtimber on timberland, by species, Florida, 1987-1994

\begin{tabular}{|c|c|c|}
\hline Species & $\begin{array}{l}\text { Net } \\
\text { annual growth }\end{array}$ & $\begin{array}{c}\text { Annual } \\
\text { timber removals }\end{array}$ \\
\hline & \multicolumn{2}{|c|}{ Thousand board feet } \\
\hline \multicolumn{3}{|l|}{ Softwood } \\
\hline Yellow pines & $1,225,727$ & $1,103,554$ \\
\hline Eastern white pine & - & - \\
\hline Spruce and fir & - & - \\
\hline Cypress & 183,096 & 112,872 \\
\hline Other Eastern softwoods & 13,388 & 1,469 \\
\hline Total softwoods & $1,422,211$ & $1,217,895$ \\
\hline \multicolumn{3}{|l|}{ Hardwood } \\
\hline Select white and red oaks & 4,018 & 4,517 \\
\hline Other white and red oaks & 254,823 & 127,522 \\
\hline Hickory & 13,987 & 12,401 \\
\hline Yellow birch & - & - \\
\hline Hard maple & 1,635 & 1,291 \\
\hline Sweetgum & 57,630 & 36,373 \\
\hline Ash, walnut, and black cherry & 18,596 & 7,240 \\
\hline Yellow-poplar & 10,768 & 3,350 \\
\hline Tupelo and blackgum & 79,706 & 33,188 \\
\hline Bay and magnolia & 58,303 & 19,200 \\
\hline Other Eastern hardwoods & 43,501 & 19,957 \\
\hline Total hardwoods & 542,967 & 265,039 \\
\hline All species & $1,965,178$ & $1,482,934$ \\
\hline
\end{tabular}


Table 43-Average annual removals of growing stock on timberland, by species and diameter class, Florida, 1987-1994

\begin{tabular}{|c|c|c|c|c|c|c|c|c|c|c|c|}
\hline \multirow[b]{2}{*}{ Species } & \multirow[b]{2}{*}{$\begin{array}{c}\text { All } \\
\text { classes }\end{array}$} & \multicolumn{10}{|c|}{ Diameter class (inches at breast height) } \\
\hline & & $\begin{array}{l}5.0 \\
6.9\end{array}$ & $\begin{array}{l}7.0 \\
8.9\end{array}$ & $\begin{array}{c}9.0 \\
10.9\end{array}$ & $\begin{array}{l}11.0- \\
12.9 \\
\end{array}$ & $\begin{array}{l}13.0 \\
14.9 \\
\end{array}$ & $\begin{array}{l}15.0 \\
16.9 \\
\end{array}$ & $\begin{array}{l}17.0- \\
18.9 \\
\end{array}$ & $\begin{array}{l}19.0 \\
20.9\end{array}$ & $\begin{array}{l}21.0 \\
28.9\end{array}$ & $\begin{array}{l}29.0 \text { and } \\
\text { larger }\end{array}$ \\
\hline & \multicolumn{11}{|c|}{ Thousand cubic feet } \\
\hline \multicolumn{12}{|l|}{ Softwood } \\
\hline Yellow pines & 431,436 & 70,019 & 122,058 & 99,723 & 58,297 & 35,849 & 21,118 & 11,467 & 5,579 & 6,946 & 380 \\
\hline Eastern white pine & - & - & - & - & - & - & - & - & - & - & - \\
\hline Spruce and fir & - & - & - & - & - & - & - & - & - & - & - \\
\hline Cypress & 40,754 & 4,359 & 8,289 & 9,210 & 8,090 & 5,039 & 2,981 & 963 & 833 & 785 & 205 \\
\hline Other Eastern softwoods & 443 & 113 & 67 & - & - & 95 & 168 & - & - & - & - \\
\hline Total softwoods & 472,633 & 74,491 & 130,414 & 108,933 & 66,387 & 40,983 & 24,267 & 12,430 & 6,412 & 7,731 & 585 \\
\hline \multicolumn{12}{|l|}{ Hardwood } \\
\hline Select white and red oaks & 1,199 & - & 89 & 163 & 146 & 237 & 75 & - & 76 & 268 & 145 \\
\hline Other white and red oaks & 39,534 & 3,767 & 4,750 & 4,566 & 4,698 & 4,561 & 4,662 & 3,496 & 2,419 & 5,252 & 1,363 \\
\hline Hickory & 3,062 & 106 & 86 & 118 & 344 & 938 & 381 & 440 & 132 & 517 & - \\
\hline Yellow birch & - & - & - & - & - & - & - & - & - & - & - \\
\hline Hard maple & 379 & - & - & 77 & 67 & 71 & - & 88 & - & 76 & - \\
\hline Sweetgum & 11,403 & 681 & 683 & 1,990 & 2,283 & 1,811 & 2,221 & 1,101 & - & 528 & 105 \\
\hline Ash, walnut, and black cherry & 3,167 & 628 & 441 & 350 & 694 & 139 & 433 & - & 298 & 184 & - \\
\hline Yellow-poplar & 1,147 & 305 & 225 & 44 & - & 73 & - & - & - & 500 & - \\
\hline Tupelo and blackgum & 13,621 & 1,440 & 2,212 & 2,309 & 1,717 & 1,876 & 1,276 & 444 & 905 & 1,442 & - \\
\hline Bay and magnolia & 7,881 & 985 & 656 & 1,548 & 1,102 & 1,233 & 902 & 575 & 424 & 356 & 100 \\
\hline Other Eastern hardwoods & 6,693 & 601 & 779 & 642 & 734 & 1,274 & 694 & 1,119 & 476 & 374 & - \\
\hline Total hardwoods & 88,086 & 8,513 & 9,921 & 11,807 & 11,785 & 12,213 & 10,644 & 7,263 & 4,730 & 9,497 & 1,713 \\
\hline All species & 560,719 & 83,004 & 140,335 & 120,740 & 78,172 & 53,196 & 34,911 & 19,693 & 11,142 & 17,228 & 2,298 \\
\hline
\end{tabular}

Table 44-Average annual mortality of live timber, growing stock, and sawtimber on timberland, by species, Florida, 1987-1994

\begin{tabular}{|c|c|c|c|}
\hline Species & Live timber ${ }^{2}$ & Growing stock & Sawtimber \\
\hline & \multicolumn{2}{|c|}{ Thousand cubic feet } & $\begin{array}{l}\text { Thousand } \\
\text { board feet }\end{array}$ \\
\hline \multicolumn{4}{|l|}{ Softwood } \\
\hline Yellow pines & 45,735 & 44,963 & 156,073 \\
\hline Eastern white pine & - & - & - \\
\hline Spruce and fir & - & - & - \\
\hline Cypress & 8,597 & 7,653 & 13,603 \\
\hline Other Eastern softwoods & 1,261 & 1,086 & 4,690 \\
\hline Total softwoods & 55,593 & 53,702 & 174,366 \\
\hline \multicolumn{4}{|l|}{ Hardwood } \\
\hline Select white and red oaks & 346 & 346 & 1,114 \\
\hline Other white and red oaks & 22,016 & 14,153 & 50,040 \\
\hline Hickory & 906 & 628 & 2,327 \\
\hline Yellow birch & - & - & - \\
\hline Hard maple & 257 & - & - \\
\hline Sweetgum & 5,161 & 4,569 & 14,021 \\
\hline Ash, walnut, and black cherry & 6,547 & 4,383 & 11,837 \\
\hline Yellow-poplar & 456 & 429 & 1,575 \\
\hline Tupelo and blackgum & 10,693 & 7,830 & 18,911 \\
\hline Bay and magnolia & 13,276 & 10,116 & 27,496 \\
\hline Other Eastern hardwoods & 23,459 & 9,556 & 29,506 \\
\hline Total hardwoods & 83,117 & 52,010 & 156,827 \\
\hline All species & 138,710 & 105,712 & 331,193 \\
\hline
\end{tabular}

${ }^{a}$ Merchantable portion only. 
Table 45-Change in number of live trees on timberland, by species group, survey completion date, and diameter class, Florida

\begin{tabular}{|c|c|c|c|c|c|c|c|c|c|}
\hline \multirow[b]{2}{*}{$\begin{array}{l}\text { Species group } \\
\text { and year }\end{array}$} & \multirow[b]{2}{*}{$\begin{array}{c}\text { All } \\
\text { classes }\end{array}$} & \multicolumn{8}{|c|}{ Diameter class (inches at breast height) } \\
\hline & & $\begin{array}{l}1.0 \\
2.9\end{array}$ & $\begin{array}{l}3.0- \\
4.9\end{array}$ & $\begin{array}{l}5.0- \\
6.9\end{array}$ & $\begin{array}{l}7.0 \\
8.9\end{array}$ & $\begin{array}{l}9.0- \\
10.9\end{array}$ & $\begin{array}{l}11.0 \\
12.9\end{array}$ & $\begin{array}{l}13.0- \\
14.9\end{array}$ & $\begin{array}{c}15.0 \text { and } \\
\text { larger }\end{array}$ \\
\hline & \multicolumn{9}{|c|}{ Thousand trees } \\
\hline \multicolumn{10}{|l|}{ Yellow pine } \\
\hline 1987 & $2,277,233$ & 777,936 & 647,330 & 429,137 & 220,314 & 97,458 & 54,748 & 28,208 & 22,102 \\
\hline 1995 & $2,635,916$ & 903,832 & 798,294 & 503,379 & 232,217 & 94,842 & 50,641 & 28,112 & 24,599 \\
\hline Change & 358,683 & 125,896 & 150,964 & 74,242 & 11,903 & $-2,616$ & $-4,107$ & -96 & 2,497 \\
\hline \multicolumn{10}{|l|}{ Other softwood } \\
\hline 1987 & 843,420 & 372,471 & 183,153 & 111,145 & 72,940 & 47,123 & 25,884 & 15,093 & 15,611 \\
\hline 1995 & 693,937 & 299,046 & 156,820 & 87,486 & 59,237 & 39,740 & 24,283 & 14,381 & 12,944 \\
\hline Change & $-149,483$ & $-73,425$ & $-26,333$ & $-23,659$ & $-13,703$ & $-7,383$ & $-1,601$ & -712 & $-2,667$ \\
\hline \multicolumn{10}{|l|}{ Hardwood } \\
\hline 1987 & $4,391,193$ & $2,793,826$ & 843,727 & 332,541 & 167,817 & 99,909 & 61,597 & 35,722 & 56,054 \\
\hline 1995 & $4,304,187$ & $2,737,863$ & 823,220 & 330,245 & 161,667 & 98,261 & 60,466 & 38,170 & 54,295 \\
\hline Change & $-87,006$ & $-55,963$ & $-20,507$ & $-2,296$ & $-6,150$ & $-1,648$ & $-1,131$ & 2,448 & $-1,759$ \\
\hline
\end{tabular}

Table 46-Land area, by land use class, major forest type, and survey completion date, Florida

\begin{tabular}{|c|c|c|c|c|}
\hline \multirow[b]{2}{*}{ Land use class } & \multicolumn{3}{|c|}{ Survey completion date } & \multirow{2}{*}{$\begin{array}{c}\text { Change } \\
1987-1995 \\
\end{array}$} \\
\hline & 1980 & 1987 & 1995 & \\
\hline & \multicolumn{4}{|c|}{ Acres } \\
\hline \multicolumn{5}{|l|}{ Forest land } \\
\hline \multicolumn{5}{|l|}{ Timberland } \\
\hline Pine and oak-pine types & $9,193,657$ & $8,737,336$ & $8,916,768$ & 179,432 \\
\hline Hardwood types & $6,470,520$ & $6,245,271$ & $5,733,892$ & $-511,379$ \\
\hline Total & $15,664,177$ & $14,982,607$ & $14,650,660$ & $-331,947$ \\
\hline Reserved timberland & 411,844 & 403,569 & 522,676 & 119,107 \\
\hline Woodland & $1,057,868$ & $1,162,836$ & $1,047,861$ & $-114,975$ \\
\hline Total forest land & $17,133,889$ & $16,549,012$ & $16,221,197$ & $-327,815$ \\
\hline \multicolumn{5}{|l|}{ Nonforest land } \\
\hline Cropland & $3,784,515$ & $3,937,202$ & $3,616,344$ & $-320,858$ \\
\hline Pasture and range & $6,991,503$ & $6,324,067$ & $5,925,691$ & $-398,376$ \\
\hline Other & $6,622,456$ & $7,721,452$ & $8,692,576$ & 971,124 \\
\hline Total & $17,398,474$ & $17,982,721$ & $18,183,805$ & 201,084 \\
\hline All land ${ }^{a}$ & $34,532,363$ & $34,531,733$ & $34,405,002$ & $-126,731$ \\
\hline
\end{tabular}

${ }^{a}$ Excludes all water areas. 
Table 47-Volume of sawtimber, growing stock, and live timber on timberland, by species group, survey completion date, and diameter class, Florida

\begin{tabular}{|c|c|c|c|c|c|c|c|c|c|c|}
\hline \multirow[b]{2}{*}{$\begin{array}{l}\text { Species group } \\
\text { and year }\end{array}$} & \multirow[b]{2}{*}{$\begin{array}{c}\text { All } \\
\text { classes }\end{array}$} & \multicolumn{9}{|c|}{ Diameter class (inches at breast height) } \\
\hline & & $\begin{array}{l}5.0- \\
6.9\end{array}$ & $\begin{array}{l}7.0- \\
8.9\end{array}$ & $\begin{array}{l}9.0- \\
10.9\end{array}$ & $\begin{array}{l}11.0- \\
12.9\end{array}$ & $\begin{array}{l}13.0- \\
14.9\end{array}$ & $\begin{array}{l}15.0- \\
16.9\end{array}$ & $\begin{array}{l}17.0 \\
18.9\end{array}$ & $\begin{array}{l}19.0 \\
20.9\end{array}$ & $\begin{array}{c}21.0 \text { and } \\
\text { larger }\end{array}$ \\
\hline
\end{tabular}

\section{Softwood}

1980
1987

1995

\section{Hardwood}

1980

1987

1995

$25,288,908$
$26,811,055$
$28,311,539$

$12,862,300$
$14,956,318$
$16,975,691$

\section{Softwood}

1980
1987
1995

1995

1980

1987

1995

$8,940,399$
$9,005,689$
$9,424,434$

$4,874,279$
$5,416,430$
$5,941,997$

-
-
-
-
-
-

-
-
-
-
-
-

SAWTIMBER (in thousand board feet)

$\begin{array}{ccccccc}5,915,872 & 6,617,620 & 5,129,410 & 3,207,653 & 1,864,608 & 1,091,351 & 1,462,394 \\ 5,638,755 & 6,213,683 & 5,424,094 & 3,636,648 & 2,311,164 & 1,413,880 & 2,172,831 \\ 5,730,769 & 6,275,825 & 5,923,818 & 4,251,003 & 2,545,662 & 1,634,593 & 1,949,869 \\ & & & & & & \\ - & 2,277,459 & 2,446,847 & 1,964,785 & 1,670,557 & 1,310,429 & 3,192,223 \\ - & 2,566,240 & 2,564,011 & 2,398,373 & 1,913,551 & 1,513,551 & 4,000,592 \\ - & 2,981,663 & 3,214,815 & 2,740,331 & 2,251,808 & 1,642,333 & 4,144,741\end{array}$

GROWING STOCK (in thousand cubic feet)

\begin{tabular}{|c|c|c|c|c|c|c|c|c|c|c|}
\hline \multicolumn{11}{|l|}{ 年 } \\
\hline 1980 & $8,940,399$ & $1,445,945$ & $1,775,379$ & $1,709,753$ & $1,547,213$ & $1,067,882$ & 617,849 & 341,641 & 192,076 & 242,661 \\
\hline 1987 & $9,005,689$ & $1,386,648$ & $1,776,304$ & $1,613,768$ & $1,432,572$ & $1,106,036$ & 683,945 & 411,512 & 241,959 & 352,945 \\
\hline 1995 & $9,424,434$ & $1,571,700$ & $1,867,428$ & $1,602,653$ & $1,416,220$ & $1,178,211$ & 776,631 & 438,859 & 269,451 & 303,281 \\
\hline \multicolumn{11}{|c|}{ Hardwood } \\
\hline 1980 & $4,874,279$ & 464,613 & 585,890 & 677,110 & 744,949 & 669,449 & 482,913 & 376,609 & 277,797 & 594,949 \\
\hline 1987 & $5,416,430$ & 518,512 & 649,543 & 750,311 & 779,320 & 667,542 & 563,875 & 419,598 & 314,177 & 753,552 \\
\hline 1995 & $5,941,997$ & 587,973 & 707,344 & 845,636 & 863,159 & 797,141 & 610,444 & 465,130 & 320,829 & 744,341 \\
\hline & \multicolumn{10}{|c|}{ LIVE TIMBER ${ }^{a}$ (in thousand cubic feet) } \\
\hline \multicolumn{11}{|c|}{ Softwood } \\
\hline 1980 & $9,088,634$ & $1,482,349$ & $1,808,645$ & $1,734,238$ & $1,561,961$ & $1,077,478$ & 624,841 & 343,672 & 193,460 & 261,990 \\
\hline 1987 & $9,131,725$ & $1,408,743$ & $1,802,601$ & $1,635,810$ & $1,444,883$ & $1,113,769$ & 691,214 & 417,415 & 244,025 & 373,265 \\
\hline 1995 & $9,525,645$ & $1,593,047$ & $1,886,820$ & $1,617,098$ & $1,425,990$ & $1,185,398$ & 782,932 & 441,972 & 270,448 & 321,940 \\
\hline \multicolumn{11}{|c|}{ Hardwood } \\
\hline 1980 & $6,470,923$ & 741,394 & 841,727 & 905,146 & 928,757 & 812,440 & 601,657 & 458,602 & 353,670 & 827,530 \\
\hline 1987 & $7,007,008$ & 779,954 & 879,800 & 962,037 & 962,438 & 804,803 & 675,937 & 511,612 & 384,867 & $1,045,560$ \\
\hline 1995 & $7,490,035$ & 851,992 & 920,663 & $1,046,380$ & $1,025,876$ & 929,492 & 734,714 & 555,242 & 400,114 & $1,025,562$ \\
\hline
\end{tabular}

${ }^{a}$ Merchantable volume. 


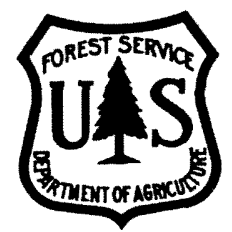

The Forest Service, U.S. Department

of Agriculture, is dedicated to the principle of

multiple use management of the Nation's forest resources for sustained yields of wood, water, forage, wildlife, and recreation. Through forestry research, cooperation with the States and private forest owners, and management of the National Forests and National Grasslands, it strives-as directed by Congress - to provide increasingly greater service to a growing Nation.

The United States Department of Agriculture (USDA) prohibits discrimination in its programs on the basis of race, color, national origin, sex, religion, age, disability, political beliefs, and marital or familial status. (Not all prohibited bases apply to all programs.) Persons with disabilities who require alternative means of communication of program information (braille, large print, audiotape, etc.) should contact the USDA office of Communications at (202) 720-2791. To file a complaint, write the Secretary of Agriculture, U.S. Department of Agriculture, Washington, DC 20250, or call (202) $720-7327$ (voice) or (202) 720-1127 (TDD). USDA is an equal employment opportunity employer. 
Brown, Mark J. 1996. Forest statistics for Florida, 1995. Resour. Bull. SRS-6. Asheville, NC: U.S. Department of Agriculture, Forest Service, Southern Research Station. $48 \mathrm{p}$.

Since 1987, area of timberland in Florida decreased by 2 percent to less than 14.7 million acres. Timberland under nonindustrial private forest ownership increased 2 percent to 7.2 million acres, and public ownership increased 16 percent to 2.8 million acres, but timberland controlled by forest industry decreased by 16 percent to 4.6 million acres. Pine plantations now account for 32 percent of the State's timberland, the highest proportion in the South. Volume of softwood growing stock increased almost 5 percent to 9.4 billion cubic feet, and volume of hardwood growing stock increased nearly 10 percent to more than 5.9 billion cubic feet. Net annual growth of softwood growing stock increased 9 percent to 532 million cubic feet, and that of hardwood increased 14 percent to 161 million cubic feet. Average annual removals of softwood growing stock changed little at 473 million cubic feet, whereas that for hardwood increased 33 percent to 88 million cubic feet.

KEYWORDS: Timberland, ownership, forest type, harvest, regeneration, growing stock, volume, growth, removals, mortality. 


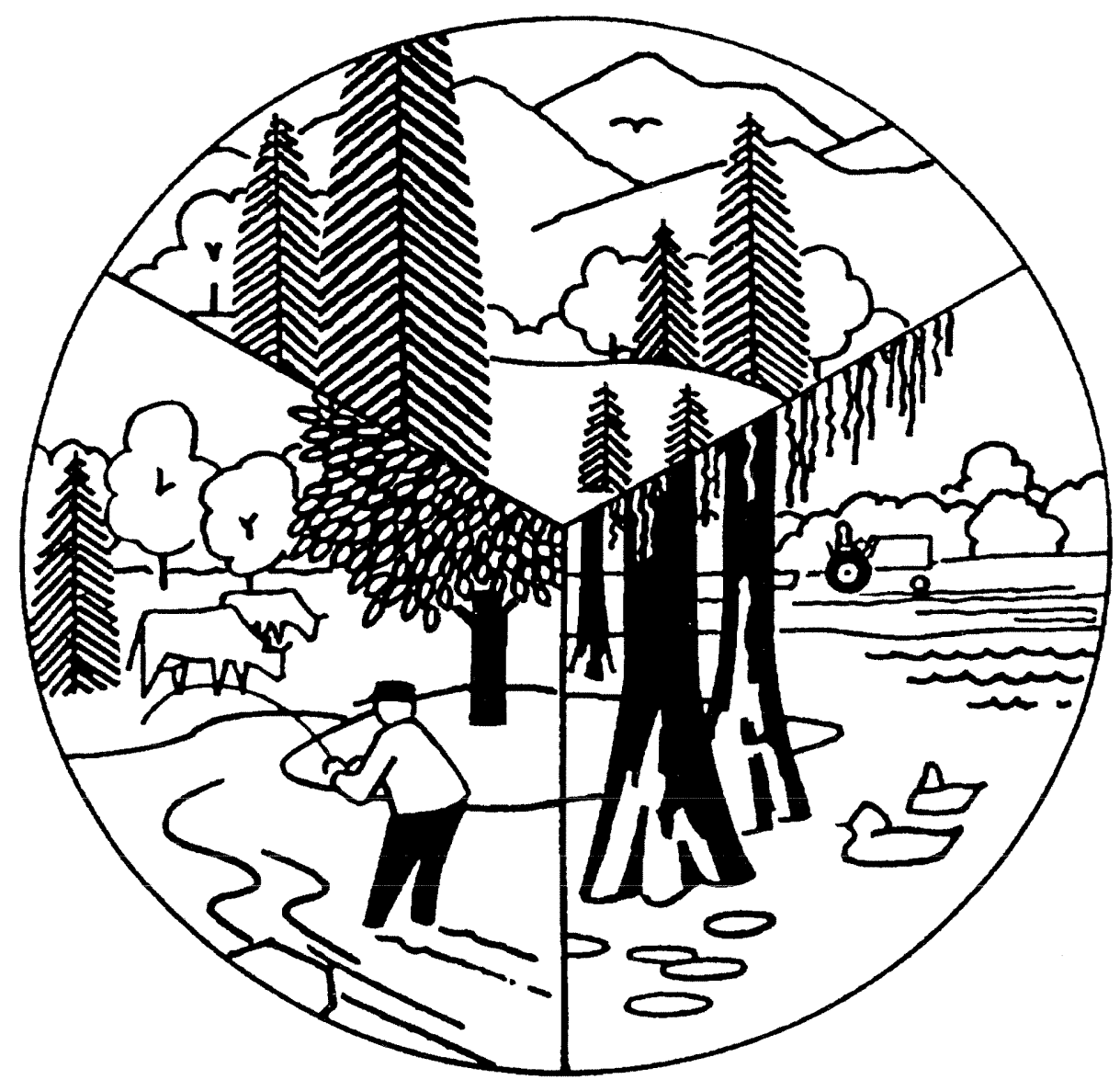

\section{Southern Research Station Established 1921}

The Southern Research Station, headquartered in Asheville, North Carolina, is one of the seven regional Stations and the Forest Products Laboratory that make up the Forest Service research organization.

\section{RESEARCH MISSION:}

To acquire the knowledge, develop the technology, and disseminate the research findings required to manage the Southern forest resources in ways that satisfy demands of goods and services while maintaining a quality environment. 